\title{
Inferring volatility dynamics and risk premia from the S\&P 500 and VIX markets *
}

\author{
Chris BardgetT $^{\dagger} \quad$ Elise Gourier $^{\ddagger} \quad$ Markus Leippold $^{\S}$
}

March 2018

\begin{abstract}
We estimate a flexible affine model using an unbalanced panel containing S\&P 500 and VIX index returns and option prices, and analyze the contribution of VIX options to the model's in- and out-of-sample performance. We find that they contain valuable information on the risk-neutral conditional distributions of volatility at different time horizons, which is not spanned by the S\&P 500 market. This information allows enhanced estimation of the variance risk premium. We gain new insights on the term structure of the variance risk premium, present a trading strategy exploiting these insights and show how to improve S\&P 500 return forecasts.
\end{abstract}

KEYWORDS: S\&P 500 and VIX joint modeling, volatility dynamics, particle filter, variance risk premium.

JEL CODES: G12, G13, C58.

${ }^{*}$ We thank G. William Schwert (the editor), an anonymous referee as well as Yacine Aït-Sahalia, Torben Andersen, Tim Bollerslev, Peter Christoffersen, Rama Cont, Jérôme Detemple, Garland Durham, Damir Filipović, Andras Fulop, René Garcia, Michael Johannes, Loriano Mancini, Ian Martin, Chay Ornthanalai, Chris Rogers, Olivier Scaillet, Ronnie Sircar, Josef Teichmann, Fabio Trojani, Anders Trolle, and Alexandre Ziegler for helpful comments. Useful feedback was received from seminar participants at University of Zürich, ETH, University of Aarhus, HEC Montreal, Queen Mary University, Kent Business School, Toulouse School of Economics, Rotman School of Management, University of Montreal, UCL, LSE and CREST as well as at the Swiss Finance Institute Gerzensee workshop, the Bachelier Finance Conference, EFA 2013, the 2014 Conference of the Swiss Society for Financial Market Research, the 2015 French Econometrics Conference at TSE, CFE 2016, the 2016 Paris Finance Meeting and the 2017 Empirical Finance Workshop at ESSEC. Financial support from the Swiss Finance Institute (SFI), Bank Vontobel, the Swiss National Science Foundation and the National Center of Competence in Research "Financial Valuation and Risk Management" is gratefully acknowledged. Finally, we are especially grateful for the detailed feedback of an anonymous referee. Any remaining errors are ours.

${ }^{\dagger}$ University of Zurich, Plattenstrasse 14, 8032 Zürich, Switzerland; tel: (+41)-44-634-4045; Email: chris.bardgett@bf.uzh.ch.

${ }^{\ddagger}$ Queen Mary University of London and Centre for Economic Policy Research (CEPR), Mile End Road, London E1 4NS, UK; Email: e.gourier@qmul.ac.uk.

§University of Zurich, Plattenstrasse 14, 8032 Zürich, Switzerland; tel: (+41)-44-634-5069; Email: markus.leippold@bf.uzh.ch. 


\section{Introduction}

Introduced by the CBOE in 1993, the VIX index non-parametrically approximates the expected future realized volatility of the S\&P 500 returns over the next 30 days. Options on the VIX started trading in 2006 and, as of today, represent a much larger market than VIX futures. By definition, the VIX index, VIX options, and S\&P 500 options are directly linked to the S\&P 500 index and all provide valuable information on the $\mathrm{S} \& \mathrm{P} 500$ returns dynamics. However, to the best of our knowledge, there has been very little effort dedicated to comparing the information these datasets contain on the distribution of the S\&P 500 returns and on the trajectory of their variance process. In this paper we aim to fill this gap and study the added information content of the VIX option market compared to the $\mathrm{S} \& \mathrm{P} 500$ market.

Our main contribution to the empirical option pricing literature is to show that VIX options contain valuable information on the dynamic properties of S\&P 500 returns, which is not spanned by S\&P 500 options and can be used for economic applications such as portfolio allocation or return forecasts. We draw this conclusion from a parametric approach, using a parsimonious and flexible affine model for returns. Our results are backed by various in- and out-of-sample tests as well as an in-depth analysis of the implied variance risk premium (VRP). We argue that VIX options allow for an enhanced representation of the VRP and of its term structure, and show that the resulting VRP can be used to form trading signals and improve predictions of S\&P 500 returns.

Jointly analyzing the dynamic properties and information content of the VIX and S\&P 500 option markets is a challenge. Not only do we need a model that is flexible enough to simultaneously accommodate the stylized facts of both markets over time, but the empirical analysis of such highly nonlinear data poses a significant computational hurdle. We develop a time-consistent estimation procedure that permits us to extract information from a large and unbalanced panel of data and estimate the trajectories of the unobserved volatility of S\&P 500 returns. This methodology goes well beyond a simple calibration exercise as it makes it possible to reconcile time series data on the S\&P 500 and VIX derivatives markets and consistently match the joint evolution of prices over time.

We model the S\&P 500 returns using an affine jump-diffusion specification that belongs to the class of Duffie, Pan, and Singleton (2000). This specification features two factors driving the variance 
process, and an additional factor driving the jump intensity. Its affine structure allows us to price S\&P 500 and VIX derivatives in semi-closed form, which is essential to analyze the returns and volatility dynamics using a large dataset of options. It also enables us to derive the VRP in closedform and to conduct a thorough analysis of its dynamic behavior and term structure.

We find that VIX options contain information on the dynamics of the S\&P 500 returns and their variance which is not spanned by S\&P 500 options, disregarding the state of the economy. More specifically, in time of market calm, VIX options do not bring any value in estimating the current state of latent factors. However, they allow better identification of the parameters of the model, thereby providing information on the conditional distributions of the underlying returns and their variance. This translates into a better pricing of VIX options, which are not well priced when not included in the estimation dataset. This observation holds both in- and out-of-sample, and is therefore not the result of over-fitting. It also holds in times of market turmoil, but then VIX options bring value in identifying the current states of latent processes as well. Furthermore, we show that adding VIX options to the estimation allows a better representation of the term structure of variance. We synthesize VIX-type of indexes from S\&P 500 options for maturities from two to six months. Our model, when estimated to a dataset which does not include VIX options, yields RMSEs which are 15 to $20 \%$ larger than the ones obtained when VIX options are included in the estimation dataset. Our results have considerable impact in terms of pricing and risk management, which rely heavily on an accurate estimation of the conditional distributions of the underlying risk factors over different time horizons.

A by-product of our estimation is the variance risk premium (VRP), which represents the compensation investors expect to receive for bearing the risk coming from stochastic fluctuations in the variance of returns over a given horizon. The VRP corresponds to the expected payoff of a variance swap. By definition, it depends on the conditional expectation of the variance of S\&P 500 returns. Due to the affine structure of our model, the VRP is available in closed-form, which enables us to address three highly debated questions in the recent literature: What are the main components driving the VRP? Can the VRP be used to form trading signals? Does the VRP have predictive power on S\&P 500 returns?

We find that the VRP is very sensitive to jumps in the returns and their variance, in particular 
when the investment horizon is short. Hence, large movements in the variance process have an immediate negative impact on the payoff of a short-term variance swap. The two variance factors are shown to have different effects on the VRP. The first factor reacts swiftly to changing market conditions and captures most of the sudden variance fluctuations, especially during market turmoil. As such, its impact on the VRP dominates for short-term investments and during turmoil periods. The second factor is more persistent and captures mid- to long-term trends of the return variance. Its impact on the VRP is most important during calm market periods and for mid- to long-term investments.

Our results on the term structure of VRP complements recent findings of Gruber, Tebaldi, and Trojani (2015) and Dew-Becker, Giglio, Le, and Rodriguez (2017). The latter authors are the first to differentiate between periods of low and high volatility, and find that the slope of the VRP term structure switches sign in periods of distress. In line with them, we find that that the VRP has a downward sloping term structure in times of market calm, but that this is no longer true during high volatility times. Our results extend theirs as we show that the term structure of VRP is no longer monotonic in times of high volatility: it has negative slope up to three to four months, and then positive slope. On a related note, Dew-Becker, Giglio, Le, and Rodriguez (2017) show that on average, investors do not price news about future volatility and are only willing to hedge against fluctuations in short-term realized volatility. We argue that the attitude of investors towards longterm volatility changes over time, and depends on market conditions.

While the usual trading strategy to reap the VRP is to buy long-term variance contracts and sell short-term contracts, ${ }^{1}$ we show that the change in the term structure of VRP can be interpreted as a trading signal to improve the gains of this strategy. Indeed, the usual long-short strategy suffers sizable punctual losses in periods of high volatility, i.e., precisely when the term structure of VRP switches sign. We propose to switch the sign of the position in forward variance as soon as the VRP term structure switches sign. Our proposed strategy achieves a Sharpe ratio of 0.77 over the period from 2006 to 2016, against 0.01 for the usual variance swap strategy.

Finally, we examine the predictive power of our model-implied VRP on S\&P 500 returns. We find that the term structure of the VRP has predictive power on S\&P 500 returns as well, in particular for

\footnotetext{
${ }^{1}$ To implement such a strategy, one can use variance swaps, see e.g., Egloff, Leippold, and Wu (2010) and Filipović, Gourier, and Mancini (2016).
} 
horizons larger than five months. Indeed, adding a measure of skewness of the VRP term structure (or, equivalently, of convexity) to the model-implied VRP level, increases the $R^{2}$ from 0.15 (resp. 0.12 ) to 0.16 (resp. 0.15) for a horizon of 5 month (resp. 7 months). This result is intuitive. Indeed, we show that the dynamics of the VRP are well described by two latent factors. Having the VRP level and a measure of its term structure allows spanning these two factors, which are shown to contain relevant information on future returns.

As we adopt a parametric approach, our results are backed up by an extensive model specification analysis. We examine different nested models to investigate the role of the various features in explaining option prices, the risk-neutral distributions of returns, and those of the variance process. Of course, any parametric approach is bound to suffer, to a certain extent, from model misspecification. Based on likelihood criteria and analyses of the in- and out-of sample pricing errors, we show that the full specification of our model is needed to represent the underlying indices as well as the options on both markets. However, we perform all tests in the paper using a benchmark two-factor affine model; all our results hold using both specifications.

We address the computational challenge of jointly estimating a model to two liquid option markets by designing an option pricing algorithm and a particle filter, which are tailored to our problem and model specification. Estimating the dynamics of the S\&P 500 returns from an extremely large dataset of options on the two markets and for a long time series requires computationally efficient techniques that can easily deal with the features of the model, in particular the state-dependent jumps. To achieve this goal, we extend the Fourier Cosine method introduced by Fang and Oosterlee (2008) for S\&P 500 options to price VIX options and adapt the Auxiliary Particle Filter of Pitt and Shephard (1999) to estimate the trajectories of unobservable processes and jumps. Accordingly, we provide an extensive toolkit for inference and diagnostics of affine option pricing models given index and option data from both the S\&P 500 and VIX markets. Particle filtering techniques and more generally Sequential Monte Carlo methods have recently increased in popularity and have been used to estimate models, but most endeavors using this tool restrict their options dataset to near at-the-money options and as far as we know, none have used S\&P 500 and VIX derivatives jointly.

Our work is related to several recent papers which aim to reconcile the cross-sectional information of the S\&P 500 and the VIX derivatives markets by modeling them jointly. Gatheral (2008) pointed 
out first that even though the Heston model performs fairly well at pricing S\&P 500 options, it fails to price VIX options. In fact, modeling the instantaneous volatility as a square root process leads to a VIX smile decreasing with moneyness, which is the opposite of what is observed in practice. Among the recent papers that have attempted to simultaneously reproduce the volatility smiles of S\&P 500 and VIX options are Chung, Tsai, Wang, and Weng (2011), Cont and Kokholm (2013), Papanicolaou and Sircar (2014), and Bayer, Gatheral, and Karlsmark (2013). We build on this literature by considering extensions of the Heston model that remain within the affine framework, but add more flexibility to the specifications used in the above mentioned papers. We use a special case of the general affine framework developed by Duffie, Pan, and Singleton (2000) that includes as sub-cases the usual extensions of the Heston model encountered in the literature, for example Bates (2000b), Eraker (2004), and Sepp (2008a). ${ }^{2}$ In related work, Song and Xiu (2016) use a model that is similar to ours but with a different focus, and estimate marginal densities and pricing kernels of the market returns and VIX. In particular, they find, interestingly, a pricing kernel of the VIX that is U-shaped, similarly to the kernel of market returns. Because our dataset in liquidly traded VIX options mainly contains calls and therefore information on the right tail of the variance distribution, we remain agnostic about the price of large downward volatility changes. In contrast to their paper where they focus on prices of risks over a 42-day horizon, we analyze the added information contents of VIX options on the entire term structure of variance, and the subsequent economic implications in terms of portfolio allocation and return forecasts.

We also build on a literature which studies the dynamic properties of variance risk premia. Amengual (2008) uses S\&P 500 options and variance swaps to infer the term structure of variance risk premia prior to the financial crisis. He finds a downward-sloping term structure of variance risk premia, which is confirmed by later studies by Andries, Eisenbach, Schmalz, and Wang (2015), solely based on S\&P 500 options. Gruber, Tebaldi, and Trojani (2015) differentiate between periods of low and high volatility, and Dew-Becker, Giglio, Le, and Rodriguez (2017) show that on average, investors do not price news about future volatility and are only willing to hedge against fluctuations in short-term realized volatility. Our results are complementary to theirs.

\footnotetext{
${ }^{2}$ Some studies are going in the direction of non-affine models (e.g., Jones (2003), Aït-Sahalia and Kimmel (2007), Christoffersen, Jacobs, and Mimouni (2010), Ferriani and Pastorello (2012), Durham (2013), Kaeck and Alexander (2012)). However, tractability remains an issue that is of crucial importance when it comes to calibrating a model to a long time series containing hundreds of options each day.
} 
Finally, our work enriches the literature on time-consistent estimation methods. These methods have been previously used to calibrate models to index returns and options. See, e.g., Bates (2000a), Pan (2002), Eraker (2004), Broadie, Chernov, and Johannes (2007), Christoffersen, Jacobs, and Mimouni (2010), Johannes, Polson, and Stroud (2009), and Duan and Yeh (2011). However, as underlined in Ferriani and Pastorello (2012), most papers filtering information from option prices rely on one option per day or a limited set of options. Limiting the amount of data results in a computationally less intensive empirical exercise, but it ignores a large part of the information present in the markets. In contrast, in our particle filter estimation we fully exploit the richness of our dataset. Furthermore, we note that most papers that consider S\&P 500 and VIX options in their calibration exercise have restricted their analysis to a static one-day estimation. The resulting parameters might exhibit large variations when calibrating the model to different dates and therefore cannot be used to infer time series properties of returns and risk premia. ${ }^{3}$

This paper is organized as follows. In Section 2, we introduce the three-factor affine jumpdiffusion framework used later in the estimation. We describe the risk premium specification, derive the expressions of the VRP and of the VIX squared as well as the pricing formula for VIX and S\&P 500 options. In Section 3, we describe our dataset. In Section 4, we detail our time series consistent estimation method. In Section 5, we discuss our estimation results and model specification analysis. Section 6 provides a thorough analysis of the VRP and the properties of its term structure. In Section 7, we discuss two economic implications of our model and estimation methodology. The first one shows how the model-implied term structure of VRP can be used as a trading signal, and the second one examines the predictive power of the VRP on future S\&P 500 returns. Section 8 concludes.

\section{Theoretical framework}

We first present our modeling framework. Our model is novel and able to capture important stylized facts of S\&P 500 returns, which have been recently highlighted in the literature. In particular, it includes a state-of-the-art representation of the jumps, inspired from Andersen, Fusari, and Todorov (2015) and Amengual and Xiu (2015), which makes it possible to better capture the stochastic skewness of returns and of their variance. Despite its flexibility, it is parsimonious and

\footnotetext{
${ }^{3}$ See, e.g., Lindström, Ströjby, Brodén, Wiktorsson, and Holst (2008).
} 
tractable as it belongs to the affine model class.

\subsection{Model specification}

Let $\left(\Omega, \mathcal{F},\left\{\mathcal{F}_{t}\right\}_{t \geq 0}, \mathbb{P}\right)$ be a filtered probability space satisfying the usual assumptions, where $\mathbb{P}$ denotes the historical measure. We consider a risk-neutral measure $\mathbb{Q}$ equivalent to $\mathbb{P}$. Let $\left(F_{t}\right)_{t \geq 0}$ be the forward price of the S\&P 500 index and $Y=\left(Y_{t}\right)_{t \geq 0}=\left(\log \left(F_{t}\right)\right)_{t \geq 0}$ the returns. The dynamics of $Y$ under $\mathbb{Q}$ are specified by

$$
\begin{aligned}
& d Y_{t}=\mu\left(v_{t^{-}}, m_{t}, u_{t}\right) d t+\sqrt{v_{t^{-}}} d W_{t}^{Y}+d J_{t}^{Y(+)}+d J_{t}^{Y(-)} \\
& d v_{t}=\kappa_{v}\left(m_{t}-v_{t^{-}}\right) d t+\sigma_{v} \sqrt{v_{t^{-}}} d W_{t}^{v}+d J_{t}^{v(+)}+d J_{t}^{v(-)} \\
& d m_{t}=\kappa_{m}\left(\theta_{m}-m_{t}\right) d t+\sigma_{m} \sqrt{m_{t}} d W_{t}^{m}
\end{aligned}
$$

where $W^{Y}, W^{v}$, and $W^{m}$ are standard Brownian motions. The processes $W^{Y}$ and $W^{v}$ are correlated with coefficient $\rho_{Y, v}$. All other Brownian motions are mutually independent.

The process $v=\left(v_{t}\right)_{t \geq 0}$ is the diffusive component of the variance of the $\mathrm{S} \& \mathrm{P} 500$ returns. The second variance factor $m=\left(m_{t}\right)_{t \geq 0}$ represents a stochastic level around which $v$ reverts. ${ }^{4}$ We refer to it as central tendency. The increments of the two processes $v$ and $m$ are instantaneously uncorrelated and $v$ and $m$ only interact via the drift term of $v$.

The processes $J_{t}^{Y(+)}, J_{t}^{Y(-)}, J_{t}^{v(+)}$, and $J_{t}^{v(-)}$ are finite activity jump processes driven by the point processes $N_{t}^{Y(+)}$ and $N_{t}^{Y(-)}$. The process $N_{t}^{Y(-)}$ (resp. $N_{t}^{Y(+)}$ ) counts negative (resp. positive) jumps in the returns. The jump processes are defined by

$$
J_{t}^{Y(+)}=\sum_{i=1}^{N_{t}^{Y(+)}} Z_{i}^{Y(+)}, J_{t}^{Y(-)}=\sum_{i=1}^{N_{t}^{Y(-)}} Z_{i}^{Y(-)}, J_{t}^{v(+)}=\sum_{i=1}^{N_{t}^{Y(-)}} Z_{i}^{v(+)}, J_{t}^{v(-)}=\sum_{i=1}^{N_{t}^{Y(+)}} Z_{i}^{v(-)},
$$

where $Z_{i}^{Y(+)}, Z_{i}^{Y(-)}, Z_{i}^{v(+)}$, and $Z_{i}^{v(-)}$ represent the random jump sizes. As suggested by the price paths of the S\&P 500 and VIX index, large negative (resp. positive) movements in equity returns

\footnotetext{
${ }^{4}$ It has already been shown that at least two factors are needed to provide an accurate description of the volatility dynamics (see, e.g., Bates (2000b), Andersen, Benzoni, and Lund (2002), Alizadeh, Brandt, and Diebold (2002), Chernov, Gallant, Ghysels, and Tauchen (2003), Christoffersen, Heston, and Jacobs (2009), Egloff, Leippold, and Wu (2010), Todorov (2010), Kaeck and Alexander (2012), Bates (2012), Johnson (2012), Mencía and Sentana (2013), Huang and Shaliastovich (2015), and Branger, Kraftschik, and Völkert (2016)).
} 
and large positive (resp. negative) movements in the variance are likely to occur at the same time. We therefore choose, in line with literature, the same point processes $N_{t}^{Y(+)}$ and $N_{t}^{Y(-)}$ to generate jumps in the asset returns and variance process $v$. The leverage effect is driven by the correlation between $W^{Y}$ and $W^{v}$ as well as the possibility of simultaneous jumps in the returns and variance. While it is standard to model positive jumps in the volatility, accounting for negative jumps has been less investigated. Amengual and Xiu (2015) show that negative jumps in volatility do occur and are usually triggered by macroeconomic announcements. We assume that the jump intensities depend linearly on levels of the diffusive latent processes $v, m$, and $u .^{5}$ The intensity of positive jumps in returns is denoted by $\lambda^{(+)}$and the intensity of negative jumps by $\lambda^{(-)}$:

$$
\lambda^{(+)}\left(v_{t^{-}}, m_{t}\right)=\lambda_{0}^{(+)}+\boldsymbol{\lambda}^{(+) \top} \mathbf{X}_{t^{-}}, \quad \lambda^{(-)}\left(v_{t^{-}}, m_{t}, u_{t}\right)=\lambda_{0}^{(-)}+\boldsymbol{\lambda}^{(-) \top} \mathbf{X}_{t^{-}},
$$

where $\mathbf{X}$ denotes the (column) vector of diffusive latent processes $\mathbf{X}_{t^{-}}=\left(v_{t^{-}}, m_{t}, u_{t}\right)^{\top}, \boldsymbol{\lambda}^{(+)}=$ $\left(\lambda_{1}^{(+)}, \lambda_{2}^{(+)}, 0\right)^{\top}$ and $\boldsymbol{\lambda}^{(-)}=\left(\lambda_{1}^{(-)}, \lambda_{2}^{(-)}, \lambda_{3}^{(-)}\right)^{\top}$. The process $u$ is an additional driver of the intensity of negative jumps in returns (together with positive jumps in variance), as in Andersen, Fusari, and Todorov (2015). It has the following dynamics:

$$
d u_{t}=\kappa_{u}\left(\theta_{u}-u_{t}\right) d t+\sigma_{u} \sqrt{u_{t}} d W_{t}^{u}
$$

with $W^{u}$ independent of the other Brownian motions. Intuitively, $u$ allows us to better represent the stochastic skewness of the return process. Andersen, Fusari, and Todorov (2015) find that the effect of the process $u$ on the intensity of positive jumps in returns and on the diffusive variance is insignificant, therefore we do not incorporate it in our model.

We assume that the random jump sizes are independent and identically distributed. For the jumps in return, we assume that positive and negative jumps are exponentially distributed with respective means $\mu_{Y}^{(+)}$and $\mu_{Y}^{(-)}$.

\footnotetext{
${ }^{5}$ The specification of jumps is of importance. Todorov (2010), Todorov and Tauchen (2011) and Jacod and Todorov (2010) find striking evidence for co-jumps in S\&P 500 returns and in the VIX. See also Eraker (2004), Broadie, Chernov, and Johannes (2007), Cont and Kokholm (2013). Bates (1996), Pan (2002) and Eraker (2004) argue in favor of using state-dependent jumps in returns, which is intuitively appealing, as jumps tend to occur more frequently when volatility increases. Using variance swaps, Aït-Sahalia, Karaman, and Mancini (2012) find that the state dependent intensity of jumps is a desirable model feature.
} 
The positive and negative jump sizes in return volatility are assumed to be exponentially distributed with mean $\nu_{v}^{(+)}$and $\nu_{v}^{(-)}$, respectively. Let us define $\mathbf{Z}_{i}=\left(Z_{i}^{Y(+)}, Z_{i}^{Y(-)}, Z_{i}^{v(+)}, Z_{i}^{Y(-)}\right)^{\top}$, $i \in \mathbb{N}^{*}$. The jump sizes are characterized by their joint Laplace transform

$$
\theta_{Z}(\phi)=\theta_{Z_{1}}\left(\phi_{Y}^{(+)}, \phi_{Y}^{(-)}, \phi_{v}^{(+)}, \phi_{v}^{(-)}\right)=\mathbb{E}^{\mathbb{Q}}\left[\exp \left(\phi^{\top} \mathbf{Z}_{1}\right)\right], \quad \phi \in \mathbb{C}^{3}
$$

The drift of the returns process can be written accordingly as:

$$
\mu\left(v_{t^{-}}, m_{t}, u_{t}\right)=-\lambda^{(-)}\left(v_{t^{-}}, m_{t}, u_{t}\right)\left(\theta_{Z}(0,1,0,0)-1\right)-\lambda^{(+)}\left(v_{t^{-}}, m_{t}\right)\left(\theta_{Z}(1,0,0,0)-1\right)-\frac{1}{2} v_{t^{-}}
$$

Since with the above specification, the model is driven by three latent processes $v, m$ and $u$, we refer to its general form as the SVJ3 model. In this SVJ3 model, the diffusive variance of returns can in theory reach zero with positive probability as well as become negative because of the negative jumps in $v$. While this is certainly a drawback, reaching zero is already possible with a standard Heston model with positive jumps only, when the Feller condition is not satisfied. Song and Xiu (2016), among others, find that the Feller condition is violated by the data. To tackle this issue, we verify in the empirical part of the paper that the estimated trajectory of the process $v_{t}$ never touches or crosses the zero boundary.

The above model specification implicitly defines the dynamics for the VIX. To derive its expression within our framework, we use the definition of the VIX as a finite sum of call and put prices that converges to the integral $\operatorname{VIX}_{t}^{2}=\frac{2}{\tau} \mathbb{E}_{t}^{\mathbb{Q}}\left[\int_{t}^{t+\tau} \frac{d F_{u}}{F_{u^{-}}}-d\left(\ln F_{u}\right)\right]$, where $\tau$ is 30 days in annual terms.

Proposition 2.1. Under the model specification given in Eqs. (1)-(7), the VIX squared at time $t$ can be written as an affine deterministic function of $v_{t}, m_{t}$, and $u_{t}$ :

$$
\begin{aligned}
V I X_{t}^{2} & =\frac{1}{\tau} \mathbb{E}_{t}^{\mathbb{Q}}\left[\int_{t}^{t+\tau} v_{u} d u+2 \sum_{i=N_{t}^{Y v}}^{N_{t+\tau}^{Y v}}\left(e^{Z_{i}^{Y}}-1-Z_{i}^{Y}\right)\right] \\
& =\alpha_{V I X^{2}} v_{t}+\beta_{V I X^{2}} m_{t}+\gamma_{V I X^{2}} u_{t}+\delta_{V I X^{2}}
\end{aligned}
$$

where the coefficients $\alpha_{V I X^{2}}, \beta_{V I X^{2}}, \gamma_{V I X^{2}}$, and $\delta_{V I X^{2}}$ are known in closed form and provided in Appendix A. 


\subsection{Benchmark model specification}

To challenge our SVJ3 model, we specify a two-factor affine jump diffusion model as a benchmark. The dynamics of $Y$ under $\mathbb{Q}$ are simplified to

$$
\begin{aligned}
& d Y_{t}=\mu\left(v_{t^{-}}, m_{t}\right) d t+\sqrt{v_{t^{-}}} d W_{t}^{Y}+d J_{t}^{Y}, \\
& d v_{t}=\kappa_{v}\left(m_{t}-v_{t^{-}}\right) d t+\sigma_{v} \sqrt{v_{t^{-}}} d W_{t}^{v}+d J_{t}^{v}, \\
& d m_{t}=\kappa_{m}\left(\theta_{m}-m_{t}\right) d t+\sigma_{m} \sqrt{m_{t}} d W_{t}^{m}
\end{aligned}
$$

In this specification, we assume that jumps in returns are normally distributed $\mathcal{N}\left(\mu_{Y}, \sigma_{Y}\right)$ and volatility does not exhibit negative jumps. The intensity of jumps loads on $v$ and $m$, but no longer loads on a separate $u$ process. We refer to the specification in Eq.(11) as benchmark model for our SVJ3 model. This model corresponds to the benchmark model used in Filipović, Gourier, and Mancini (2016), which they estimate using variance swaps. It subsumes many of the popular stochastic volatility models as special cases, such as Bakshi, Cao, and Chen (1997), Bates (2000b, 2006), Pan (2002), Eraker, Johannes, and Polson (2003), and Broadie, Chernov, and Johannes (2007, 2009).

\subsection{Risk premium specification}

We specify the change of measure from the pricing to the historical measure so that the model dynamics have the same structure under $\mathbb{P}$. The premium for equity risk $\gamma_{t}$ consists of a diffusive contribution, which is proportional to the variance level and represents the compensation for the diffusive price risk, and a jump contribution reflecting the compensation for jump risk:

$\gamma_{t}=\eta_{Y} v_{t^{-}}+\lambda^{(+)}\left(v_{t^{-}}, m_{t}\right)\left(\theta_{Z}^{\mathbb{P}}(1,0,0,0)-\theta_{Z}(1,0,0,0)\right)+\lambda^{(-)}\left(v_{t^{-}}, m_{t}, u_{t}\right)\left(\theta_{Z}^{\mathbb{P}}(0,1,0,0)-\theta_{Z}(0,1,0,0)\right)$,

where $\theta_{Z}^{\mathbb{P}}$ denotes the joint Laplace transform of jump sizes under the historical measure $\mathbb{P}$. We follow Pan (2002) and Eraker (2004) and assume that the intensity of jumps is the same under $\mathbb{Q}$ and $\mathbb{P}^{6}$ However, we allow the mean of the jump sizes in returns to be different under $\mathbb{Q}$ and $\mathbb{P}$.

\footnotetext{
${ }^{6} \mathrm{Pan}(2002)$ argues that introducing different intensities of jumps under the historical and pricing measure introduces a jump-timing risk premium that is very difficult to disentangle from the mean jump risk premium. Our assumption
} 
Similarly, the instantaneous premium for volatility risk decomposes into a diffusive component and a jump component, for each of the volatility factors $v$ and $m$. The diffusive premium in $v$ is proportional to the current level of variance, with coefficient of proportionality given by $\eta_{v}=\kappa_{v}-\kappa_{v}^{\mathbb{P}}$. The same applies to the central tendency $m$, for which the coefficient is defined as $\eta_{m}=\kappa_{m}-\kappa_{m}^{\mathbb{P}}$. For the jump part of the premium, we allow the mean jump sizes $\nu_{v}^{(+)}$and $\nu_{v}^{(-)}$to be different under $\mathbb{P}$ and $\mathbb{Q}$.

In line with Andersen, Fusari, and Todorov (2015), we find in the empirical part that the trajectory of the factor $u$ is relatively difficult to estimate. Therefore, to avoid unnecessary complexity to the model, we assume that it does not carry any risk premium.

\subsection{Integrated variance risk premium}

Following Bollerslev and Todorov (2011), we define the annualized integrated variance risk premium (VRP) as

$$
\operatorname{VRP}(t, T)=\frac{1}{T-t}\left[\mathbb{E}_{t}^{\mathbb{P}}\left(Q V_{[t, T]}\right)-\mathbb{E}_{t}^{\mathbb{Q}}\left(Q V_{[t, T]}\right)\right]
$$

where $Q V_{[t, T]}$ denotes the quadratic variation of the log price process, which is the sum of the integrated variance of the returns and the squared jumps in the time interval considered:

$$
Q V_{[t, T]}=\int_{t}^{T} v_{s} d s+\sum_{i=N_{t}^{(+)}}^{N_{T}^{(+)}}\left(Z_{i}^{(+)}\right)^{2}+\sum_{i=N_{t}^{(-)}}^{N_{T}^{(-)}}\left(Z_{i}^{(-)}\right)^{2}
$$

The VRP represents the expected payoff when buying a variance swap at time $t$ with maturity T. Alternatively, it reflects the amount investors are willing to pay for a hedge against future stochastic fluctuations in the variance. We can further decompose the VRP into a continuous and a discontinuous part:

$$
\operatorname{VRP}(t, T)=\operatorname{VRP}^{c}(t, T)+\operatorname{VRP}^{d}(t, T)
$$

artificially incorporates the jump-timing risk premium into the mean jump size risk premium. 
with

$\operatorname{VRP}^{c}(t, T)=\frac{1}{T-t}\left[\mathbb{E}_{t}^{\mathbb{P}}\left(\int_{t}^{T} v_{s^{-}} d s\right)-\mathbb{E}_{t}^{\mathbb{Q}}\left(\int_{t}^{T} v_{s^{-}} d s\right)\right]$

$\operatorname{VRP}^{d}(t, T)=\frac{1}{T-t}\left[\mathbb{E}_{t}^{\mathbb{P}}\left(\sum_{i=N_{t}^{(+)}}^{N_{T}^{(+)}}\left(Z_{i}^{(+)}\right)^{2}+\sum_{i=N_{t}^{(-)}}^{N_{T}^{(-)}}\left(Z_{i}^{(-)}\right)^{2}\right)-\mathbb{E}_{t}^{\mathbb{Q}}\left(\sum_{i=N_{t}^{(+)}}^{N_{T}^{(+)}}\left(Z_{i}^{(+)}\right)^{2}+\sum_{i=N_{t}^{(-)}}^{N_{T}^{(-)}}\left(Z_{i}^{(-)}\right)^{2}\right)\right]$.

Each part can also be decomposed into linear contributions from $m_{t}, v_{t}$ and $u_{t}$, given that the expectations of the integrated latent factors are affine in their current values, see Appendix $\mathrm{H}$.

\subsection{Derivatives pricing}

Within the class of affine models, option pricing is most efficiently performed using Fourier inversion techniques. As a starting point, we need the characteristic function of the underlying processes. Due to the affine property of the VIX square in Proposition 2.1, we have the following result:

Proposition 2.2. In the SVJ3 model defined by Eqs. (1)-(7), the Laplace transforms of VIX ${ }^{2}$ and the $S \& P 500$ returns are exponential affine in the current values of the factor processes $v, m$ and $u$ :

$$
\begin{aligned}
& \Psi_{V I X_{T}^{2}}(t, \tilde{x} ; \omega):=\mathbb{E}_{t}^{\mathbb{Q}}\left[e^{\omega V I X_{T}^{2}} \mid \mathbf{X}_{t}=\tilde{x}\right]=e^{\alpha(T-t)+\mathbf{B}(T-t) \tilde{x}} \\
& \Psi_{Y_{T}}(t, y, \tilde{x} ; \omega):=\mathbb{E}_{t}^{\mathbb{Q}}\left[e^{\omega Y_{T}} \mid y_{t}=y, \mathbf{X}_{t}=\tilde{x}\right]=e^{\alpha_{Y}(T-t)+\beta_{Y}(T-t) y+\mathbf{B}_{\mathbf{Y}}(T-t) \tilde{x}}
\end{aligned}
$$

where $\alpha, \alpha_{Y}, \beta_{Y}, \mathbf{B}=(\beta, \gamma, \delta)^{\top}$, and $\mathbf{B}_{\mathbf{Y}}=\left(\gamma_{Y}, \delta_{Y}, \xi_{Y}\right)^{\top}$ are functions defined on $[0, T]$ by the ODEs given in Appendix B. The parameter $\omega$ belongs to a subset of $\mathbb{C}$ where the above expectations are finite.

Pricing options on the VIX poses technical difficulties that are not encountered when pricing equity options. Given a call option with strike $K_{\mathrm{VIX}}$ and maturity $T$ on the VIX at time $t=0$, we need to calculate

$$
C\left(\operatorname{VIX}_{0}, K_{\mathrm{VIX}}, T\right)=e^{-r T} \int_{0}^{\infty}\left(\sqrt{v}-K_{\mathrm{VIX}}\right)^{+} f_{\mathrm{VIX}_{T}^{2}}(v) d v
$$


where $f_{\mathrm{VIX}}^{2}$ is the $\mathbb{Q}$-density of the VIX square at time $t=T$. The square root appearing in the integral as part of the payoff in Eq. (14) prevents us from using the Fast Fourier Transform of Carr and Madan (1999). We would need the log of the VIX to be affine, which is incompatible with affine models for log-returns. However, this problem can be circumvented. Fang and Oosterlee (2008) introduce the Fourier cosine expansion to price index options on the S\&P 500. We extend their method to tackle the pricing of VIX options. Our approach to pricing VIX options is comparable to the inversion performed by Sepp (2008a) and Song and Xiu (2016), but it is more parsimonious in the number of computational parameters.

Proposition 2.3. Consider a European-style contingent claim on the VIX index with maturity $T$ and payoff $u_{V I X}\left(V I X^{2}\right)=\left(\sqrt{V I X^{2}}-K_{V I X}\right)^{+}$. Given an interval $\left[a_{V I X}, b_{V I X}\right]$ for the support of the $V I X_{T}^{2} \mid \mathbf{x}_{0}$ density, the price $P_{V I X}\left(t_{0}, V I X_{0}\right)$ at time $t=t_{0} \geq 0$ of the contingent claim is approximated by:

$$
P_{V I X}\left(t_{0}, V I X_{0}\right) \approx e^{-r\left(T-t_{0}\right)} \sum_{n=0}^{N-1} A_{n}^{V I X^{2}} U_{n}^{V I X^{2}}
$$

where the prime superscript in the sum $\sum^{\prime}$ means that the first term $A_{0}^{V I X^{2}} U_{0}^{V I X^{2}}$ is divided by two and $N$ is a truncation threshold. The terms in the sum are defined by

$$
\begin{aligned}
A_{n}^{V I X^{2}} & =\frac{2}{b_{V I X}-a_{V I X}} \operatorname{Re}\left\{\Psi_{V I X_{T}^{2}}\left(t_{0}, \mathbf{X}_{0} ; \frac{i n \pi}{b_{V I X}-a_{V I X}}\right) \exp \left(-i a_{V I X} \frac{n \pi}{b_{V I X}-a_{V I X}}\right)\right\}, \\
U_{n}^{V I X} X^{2} & =\int_{a_{V I X}}^{b_{V I X}} u_{V I X}(v) \cos \left(n \pi \frac{v-a_{V I X}}{b_{V I X}-a_{V I X}}\right) d v .
\end{aligned}
$$

The coefficient $A_{n}^{\mathrm{VIX}}{ }^{2}$ is computed using Proposition 2.2 and $U_{n}^{\mathrm{VIX}^{2}}$ is known in closed form and given in Appendix C.

\section{Data and preliminary analysis}

In this section, we describe our data and point out some important characteristics of VIX options. 


\subsection{Data description}

Options on the VIX were introduced in 2006. Our sample period is from March 1, 2006 to April 30, 2016. The option data consist of the weekly ${ }^{7}$ closing prices of European options on the S\&P 500 and VIX, obtained from OptionMetrics. This time series includes both periods of calm and periods of crisis with extreme events.

Both the S\&P 500 and VIX options datasets are treated following the literature, see e.g. AitSahalia and Lo (1998). We only consider options with maturities between one week and one year and delete options quotes that are not traded on a given date. Then, we infer from highly liquid options the futures price using the at-the-money (ATM) put-call parity. By doing so, we avoid two issues: Making predictions on future dividends and using futures closing prices which are not synchronized with the option closing prices. Hence, we consider that the underlying of the options is the index futures and not the index itself. We only work with liquid out-of-the-money (OTM) options for the S\&P 500 market and only with liquid call options for the VIX market. If the VIX in-the-money (ITM) call is not liquid, we use the put-call parity to infer a liquid VIX ITM call from a more liquid VIX OTM put. Finally, implied volatilities are computed considering futures prices as underlying. ${ }^{8}$

These adjustments leave a total of 365,507 OTM S\&P 500 and 44,539 call options on the VIX, with a daily average of 639 S\&P 500 options and 78 VIX options. The number of S\&P 500 (resp. VIX) options in our dataset on a given date increases with time, with around 170 (resp. 5) options at the beginning of the dataset and around 2,000 (resp. 160) options at the end. At the beginning of the sample, there are one or two short maturities (less than six months) available for VIX options and around six maturities for S\&P 500 options, with approximately 40 S\&P 500 options per maturity slice. At the end of the sample, the VIX options have around eight short maturities with a bit more than 20 options trading per maturity. For S\&P 500 options, around 25 maturities are available per day with around 130 options for one-month maturities and 40 options for the one-year slice. The low number of VIX options compared to the number of S\&P 500 options comes from the fact that VIX

\footnotetext{
${ }^{7}$ We follow Pan (2002) and Johannes, Polson, and Stroud (2009), among others, and use weekly (Wednesday) options data. This eliminates beginning-of-week and end-of-week effects and reduces the computational burden of the estimation.

${ }^{8}$ We remark that VIX option prices do not satisfy no-arbitrage relations with respect to the VIX index, but rather with respect to the VIX futures value. A VIX call option at time $t$ with maturity $T$ is an option on the volatility for the time interval $[T, T+30 d]$, where $30 d$ stands for 30 days. The value $\operatorname{VIX}_{t}$ at time $t$ is related to the volatility on the time interval $[t, t+30 d]$, which might not overlap at all with $[T, T+30 d]$.
} 
options only started trading in 2006. In 2010, the total VIX options volume per day is about half of the total volume of S\&P 500 options traded, and at the end of our sample it is close to two-thirds. Options on both markets are hence liquidly traded.

\subsection{Descriptive statistics}

Table 1 presents the first four sample moments of the S\&P 500 futures returns, VIX index returns and square levels over two different periods of time. The first period starts in March 2006 and ends in February 2009, i.e., it spans the pre-crisis period as well as the beginning of the crisis. The second period begins in March 2009 and lasts until April 2016. For our estimation, these two periods serve as the in-sample and out-of-sample periods.

The S\&P 500 returns exhibit a high kurtosis, especially during the in-sample period, suggesting the presence of rare and large movements. In the in-sample period, their skewness is strongly negative due to the substantial losses made during the financial crisis. It remains slightly negative during the out-of-sample period. The VIX index exhibits a large positive skewness and kurtosis both in the insample and in the out-of-sample periods, although in the latter, both statistics decrease significantly.

[Table 1 about here.]

Panel A of Figure 1 displays the joint evolution of the S\&P 500 and the VIX index from 2006 to 2016. The S\&P 500 returns and the VIX daily increments are highly negatively correlated (with a correlation coefficient of -0.589 over this period), which explains the popularity of VIX contracts for hedging part of the equity risk of a portfolio.

These time series illustrate the variety of market situations covered by our time period. Both time series are rather stable until the summer of 2007. The S\&P 500 grows almost steadily from 1200 to 1500 , whereas the VIX level is around 10-15\%. When the crisis of the quant-strategy hedge funds starts in the summer of 2007, the S\&P 500 starts falling, whereas the VIX becomes more volatile and oscillates quickly around 20\%. The bail-out of Lehman Brothers in September 2008 accelerates the crash in the S\&P 500 market, together with a large increase in the VIX index. The S\&P 500 then starts a steady increase back to its original level and above, despite some punctual mini-crashes. The VIX, in contrast, reacts much more drastically to new information. Following the 
bankruptcy of Lehman Brothers in September 2008 and the sudden increase to more than 80\%, it goes down quickly but then up again when the House of Representatives rejects the Troubled Asset Relief Program at the end of September. It then reverts back to around 40\%, but increases again following the distress of Bank of America in 2009. It gradually goes back to a level that is close to its initial level, to reach around 15\% early 2010. The flash crash in May 2010 then leads to new heights, with a peak around 45\%, and a reversion to around 10\%. In 2011, the US debt downgrade, together with the Greek financial crisis, trigger a new peak in the VIX that is reversed following the agreement on a rescue plan. The last important peak in our time series is triggered by the Chinese Yuan devaluation in May 2015.

[Fig. 1 about here.]

Even though the S\&P 500 and VIX markets are closely related, we emphasize that options on the VIX and S\&P 500 substantially differ in their characteristics and in the information they contain on the underlying S\&P 500 returns and variance. First, S\&P 500 and VIX derivatives with the same maturity contain information on the S\&P 500 over different time periods. While an S\&P 500 option with maturity $T$ contains information about the future S\&P 500 index level at time $T$ and therefore about the S\&P 500 volatility up to $T$, a VIX option with maturity $T$ embeds information about the VIX at time $T$ and therefore about the S\&P 500 volatility between $T$ and $T+30$ days. Second, the two types of options also differ in their contents. While S\&P 500 options, assuming a continuous range of traded strikes, characterize the conditional density of future S\&P 500 returns, they only provide us with a point estimate of the conditional variance of returns at each traded maturity. In turn, VIX options characterize the whole conditional density of future VIX levels. As such, they are likely to contain more information on the future density of S\&P 500 return variance.

Panels B and C of Figure 1 display the S\&P 500 and VIX smiles on May 10, 2010. The implied volatilities (IVs) are computed using the standard Black-Scholes formula. The VIX IVs are in general substantially higher than the S\&P 500 IVs. They range in our sample from $34 \%$ to $216 \%$ with an average of $80 \%$ whereas the S\&P 500 IVs range from $6 \%$ to $162 \%$ with an average of $26 \%$. The implied volatilities of S\&P 500 options are in general decreasing with moneyness, which highlights the expensiveness of out-of-the money put options on the S\&P 500. As these options provide investors 
with protection against large downward movements in returns, the negative skewness of the volatility smile reflects their risk aversion towards such movements. Due to the leverage effect, negative changes in returns are strongly related to increases in volatility, which out-of-the-money VIX call options can hedge. This explains why VIX implied volatilities tend to be positively skewed. A related quantity is the put-call trading ratio. Almost twice as many puts as calls are traded daily in the S\&P 500 options market, but the situation is reversed in the VIX market, where the amount of calls traded daily is almost double that of the puts. In fact, we can observe in Panels B and C of Figure 1 that the log-moneynesses traded for S\&P 500 options are mostly negative (which corresponds to OTM put options) and often positive for VIX options (OTM calls).

\section{Estimation methodology}

Before we bring our model to the time series of data, we carry out a joint calibration exercise using the cross-section of S\&P 500 and VIX options on specific dates. This exercise gives us some guidance for model design and allows us to reduce the set of models to be estimated on a time series of options' data. Our methodology and detailed results are reported in Appendix D.

To achieve a more robust estimation, consistent with the whole time series of in-sample data, we choose a methodology based on particle filtering. A particle filter uses a time series of observable market data, called measurements, to estimate the conditional densities of unobserved latent processes such as the volatility and jump processes at every point in time during the estimation period. It can be combined with maximum likelihood estimation for parameter estimation and standard error calculations. Using a time series of S\&P 500 and VIX indexes and options, we estimate both the $\mathbb{P}$ - and $\mathbb{Q}$-dynamics of the model to obtain a set of model parameters that jointly prices spot and options in both markets consistently over time. The estimation is performed over the in-sample period. The out-of-sample analysis is conducted by setting the parameters equal to their in-sample estimates and running the filter on the subsequent period. 


\subsection{Discretized model and specification of errors}

The state space model is obtained by discretizing the continuous-time model under $\mathbb{P}$ on a uniform time grid in time. Measurements comprise the S\&P 500 daily log-returns, the VIX levels, and the option prices on both indexes. The VIX and option prices are assumed to be observed with error. Indeed, Jiang and Tian (2007) point to systematic biases in the calculation of the VIX index, such as model misspecification or data limitations. For example, in practice, the index is calculated using a finite number of options thereby inducing an error in the computation of the integral defining VIX ${ }^{2}$. Regarding option prices, the error term represents several sources of noise, such as bid-ask spreads, timing, and processing errors.

To better identify the total variance of S\&P 500 returns under the $\mathbb{P}$ measure, we add a measurement equation, which links the logarithm of the daily Realized Variance $\left(\mathrm{RV}_{t}\right)$ of $\mathrm{S} \& \mathrm{P} 500$ returns $^{9}$ to the logarithm of the total spot variance under $\mathbb{P}$, as in Filipović, Gourier, and Mancini (2016). The associated measurement error $\epsilon_{t}$ is conditionally normally distributed with mean $\rho_{\epsilon} \epsilon_{t-1}$ and variance $c_{0}+c_{1} \mathrm{RV}_{t-1}$. The rationale behind this component of the measurement equation is the following. Andersen, Bollerslev, Diebold, and Ebens (2001), among others, provide empirical evidence that $\log \left(\mathrm{RV}_{t}\right)$ is approximately normally distributed. The conditional mean specification of $\epsilon_{t}$ allows for autocorrelation in the measurement error, which can be induced by clustering of price jumps caused by persistence of the price jump intensity and/or microstructure noise in the estimates of daily realized variance. Autocorrelation in the measurement error is also reported in Wu (2011). The conditional variance specification of $\epsilon_{t}$ captures in a parsimonious way the heteroscedasticity of the measurement error due to the volatility of realized variance.

Details on the state space model are provided in Appendix F.1.

\subsection{Particle filter}

At every discrete point in time $t=t_{n}$, the measurement vector $y_{t}$ collects observed market prices. By $y^{t}=\left(y_{t_{0}}, \ldots, y_{t_{n}}\right)$, we denote all the observations available up to time $t$. The filtering problem

\footnotetext{
${ }^{9}$ The Realized Variance (RV) of the S\&P 500 index is obtained from the website of the Oxford-Man Institute Realized Library.
} 
consists of recursively approximating the distribution of the latent state $L_{t}$,

$$
L_{t}=\left\{v_{t}, m_{t}, u_{t}, \Delta N_{t}^{(+)}, \Delta N_{t}^{(-)}, Z_{t}^{Y^{(+)}}, Z_{t}^{Y^{(-)}}, Z_{t}^{v^{(+)}}, Z_{t}^{v^{(-)}}\right\}
$$

conditional on $y^{t}$. Particle filters are perfectly adapted to our problem. They can handle observations that are nonlinear functions of latent variables as well as equations with non-Gaussian innovations.

There are many types of particle filters. We use the Auxiliary Particle Filter (APF) proposed by Pitt and Shephard (1999). Compared to more basic particle filters, such as the Sampling Importance Resampling (SIR) filter, the APF is better suited to detect jumps, whereas the SIR filter faces sample impoverishment leading to potential particle degeneracy. Both filters are described in Johannes, Polson, and Stroud (2009) for filtering latent factors from returns in a Heston model with jumps in returns.

We develop an extension of their algorithm that is able to handle more data (the VIX market data on top of the S\&P 500 market data) as well as the second volatility factor $m$, the third factor for jumps $u_{t}$ and the volatility jumps. The likelihood estimation and particle filter are presented in detail in Appendix F. In particular, we use the weighted likelihood method of $\mathrm{Hu}$ and Zidek (2002) to assign comparable weights to S\&P 500 and VIX options. Furthermore, we performed additional data treatments for S\&P 500 and VIX options before running the particle filter. They are described in Appendix F.2.

\section{Estimation results with and without VIX options}

This section discusses the choice of the full model specification and analyzes how the model performs in representing the data, depending on whether we include VIX options in the estimation dataset or not.

\subsection{Model selection}

Let us start by noting that options are crucial for identifying the parameters of our model. Even when estimating highly restricted sub-specifications of our full specification to a dataset without options (with the underlying S\&P 500 and VIX indices only), we find that the resulting estimates 
of the $\mathbb{Q}$-parameters (and some of the $\mathbb{P}$-parameters) have extremely large standard errors, typically four to five times larger than the ones obtained with datasets containing options. This problem can partly be resolved by extending the in-sample time period, leading to a more accurate estimation of the $\mathbb{P}$-parameters, but not of the $\mathbb{Q}$-parameters. Therefore, the VIX index does not contain sufficient information to identify the $\mathbb{Q}$-dynamics of S\&P 500 returns, as has been argued in, e.g., Duan and Yeh $(2010,2011) \cdot{ }^{10}$

[Table 2 about here.]

Table 2 reports the log-likelihood across the model sub-specifications as well as for the benchmark model and the values of the Akaike Information Criterion (AIC) and Bayes Information Criterion (BIC), with both estimation datasets. Inspection of these values when we include VIX options (w/ VIX options) suggests that the full SVJ3 specification is substantially superior to all nested sub-specifications examined. When VIX options are not part of the estimation dataset (w/o VIX options), the SVJ3 model only slightly out-performs the SVJ2 model, where the intensity of jumps does not load on the additional factor $u$. Given that this factor controls for simultaneous jumps in the S\&P 500 and VIX index, VIX options contain information that helps identify the parameters driving its dynamics. Therefore, the difference in performance between the SVJ2 and SVJ3 models is larger when VIX options are included in the estimation. Other sub-specifications restricting $m$ to a constant, or without jumps, significantly under-perform the SVJ2 and SVJ3 models, irrespective of whether or not we include VIX options in the data. The benchmark model, in which positive jumps in the returns are modeled together with negative jumps using a normal distribution, also under-performs the SVJ3 model, slightly when excluding and substantially when including VIX options.

[Table 3 about here.]

Table 3 presents the point estimates and standard errors resulting from the estimation of the SVJ3 model to datasets when either excluding or including VIX options. The estimated parameters driving the two variance processes allow identifying very different roles. Indeed, $v$ has a high volatility

\footnotetext{
${ }^{10}$ Results have not been reported for space constraints but are available upon request.
} 
parameter $\sigma_{v}$ ranging from 0.65 to 0.69 depending on the model specification, with small standard error. Besides, it has a high speed of mean reversion under both measures, implying a half life around 33 days under $\mathbb{P}$ and 48 days under $\mathbb{Q}$. In contrast, $m$ has a volatility parameter $\sigma_{m}$ around 0.10 regardless of the estimation dataset and chosen model, also with small standard error. Its speed of mean reversion is difficult to estimate precisely but ranges between 0.13 and 0.24 , leading to a half life to three to seven years depending on the estimation dataset. We can interpret the process $v$ as a factor representing erratic short-term fluctuations of the variance, whereas the process $m$ is persistent and captures smoother medium- to long-term trends. ${ }^{11}$ The estimated volatility of the jump process $u, \sigma_{u}$, is close to 0.30 , which suggests that $u$ is not as volatile as $v$ but also not as persistent as $m$. Its high speed of mean reversion, corresponding to a half life between 60 and 75 days, indicates that it captures punctual events. Not surprisingly, we find a prominent leverage coefficient $\rho_{Y v}$ across all models and datasets.

Similarly, Table 4 presents the point estimates and standard errors resulting from the estimation of the benchmark model to datasets that either exclude or include VIX options.

[Table 4 about here.]

Tables 5 and 6 report the Root Mean Square Relative Errors (RMSREs) of the model implied volatilities using, respectively, the SVJ3 and the benchmark model. All statistics are given for both S\&P 500 and VIX options, in- and out-of-sample, using the datasets with or without VIX options. Options are sorted into buckets by moneyness and maturity.

[Table 5 about here.]

[Table 6 about here.]

\footnotetext{
${ }^{11}$ Indeed, under the assumption that jumps have a minor impact on this expectation compared to the drift term, i.e., $\kappa_{v}^{\mathbb{P}} \gg \lambda_{k}^{(+/-)} \nu_{v}^{\mathbb{P}}$ (inequalities satisfied by our parameter estimates), the conditional expectation of the variance $\mathbb{E}_{t}^{\mathbb{P}}\left[v_{T}\right]$ can be written as:

$$
\mathbb{E}_{t}^{\mathbb{P}}\left[v_{T}\right] \approx \theta_{m}^{\mathbb{P}} \frac{\kappa_{v}}{\kappa_{v}^{\mathbb{P}}}+c \cdot e^{-\kappa_{m}^{\mathbb{P}}(T-t)}\left(m_{t}-\theta_{m}^{\mathbb{P}}\right)+e^{-\kappa_{v}^{\mathbb{P}}(T-t)}\left(v_{t}-c \cdot m_{t}+c \cdot \frac{\kappa_{m}^{\mathbb{P}}}{\kappa_{v}^{\mathbb{P}}} \theta_{m}^{\mathbb{P}}\right),
$$

for a constant $c=\frac{\kappa_{v}}{\kappa_{v}^{\mathbb{P}}-\kappa_{m}^{\mathbb{P}}}$. As $\kappa_{v}^{\mathbb{P}}>>\kappa_{m}^{\mathbb{P}}$, the coefficients in front of $v_{t}$ decays much faster than the one in front of $m_{t}$. For $T-t$ equal to three months, $e^{-\kappa_{v}^{\mathbb{P}}(T-t)}$ is around 0.16 , but goes down to 0.03 for six months, and is of order of magnitude of $10^{-3}$ for a year. In contrast, $e^{-\kappa_{m}^{\mathbb{P}}(T-t)}$ is around 0.90 for $T-t$ equal to six months, and as high as 0.80 for one year. Therefore the deviation of $m_{t}$ relative to its long-term mean drives the medium- to long-term expectation of the variance.
} 
The pricing errors of the SVJ3 model are most of the time lower than those of the benchmark model. In particular, the SVJ3 model provides a better fit to deep out-of-the-money call options on the S\&P 500 (RMSRE around 16\% for the benchmark model against 11\% for the SVJ3 model), and to long-maturity options with horizon above two months. In fact, in the benchmark model, RMSREs increase with time-to-maturity, from $10 \%$ for options with maturity below two months up to close to $15 \%$ for when the maturity is larger than six months, when excluding VIX options (from $11 \%$ to $13 \%$ when including VIX options). With the SVJ3 model, the RMSRE remains around 10\% irrespective of the time-to-maturity.

However, there are some cases when the benchmark model outperforms the SVJ3 model. For example, this is the case for deep out-of-the-money call options on the S\&P 500 out-of-sample, with an RMSRE of $11 \%$ for the benchmark model against close to $14 \%$ for the SVJ3 model. Therefore, we will conduct all the coming tests and study the economic implications using both models, to ensure our results are robust to the chosen model specification.

\subsection{Is the information in VIX options spanned by S\&P 500 options?}

Comparing RMSREs of S\&P 500 options in the two scenarios where i) we exclude VIX options from the estimation dataset and ii) we include them, reveals that values are only marginally smaller when they are excluded (0.096 versus 0.099 over all S\&P 500 options in the in-sample period). Constraining the model to fit VIX option prices therefore does not significantly affect the fit to S\&P 500 option prices. This observation is valid both outside and during the financial crisis and suggests that VIX options, in-sample, do not contain information that is conflicting with the information already spanned by S\&P 500 options. In fact, Root Mean Square Errors (RMSEs) ${ }^{12}$ even decrease from 0.044 to 0.037 when we do not include VIX options in the estimation. ${ }^{13}$ The improvement is most noticeable for deep OTM put options (RMSEs decrease from 0.102 to 0.086), OTM put options (0.027 versus 0.034$)$ and long-term options (0.032 versus 0.039), suggesting that VIX options help to

\footnotetext{
${ }^{12}$ RMSEs emphasize expensive options, i.e., options which are closer to the ATM level. For this reason we report RMSREs. RMSEs are available upon request.

${ }^{13}$ Our results are comparable to those obtained by Andersen, Fusari, and Todorov (2015) who fit a three factor volatility model to S\&P 500 options. They consider IV RMSEs as the distance to minimize and obtain an RMSE of 1.7\%. Excluding the financial crisis from the calculation of the RMSEs, we obtain RMSEs of $1.3 \%$, and $3.7 \%$ when including the crisis period. In addition, their model has an additional volatility factor hence adding flexibility to fit more data.
} 
improve the identification of model parameters driving the prices of these options. Intuitively, VIX options help pin-point stark volatility increases and provide information on the long-term behavior of variance. Out-of-sample pricing errors on S\&P 500 options confirm this intuition, as including VIX options allows to slightly improve both RMSREs and RMSEs overall, the strongest improvement being for OTM put options.

Much more interesting is the comparison of pricing errors on VIX options using our different datasets. VIX options are not well priced when they are not in the estimation dataset, i.e., the information they contain is not spanned by SEP 500 options. This is true in- and out-of sample, outside and during the crisis period. In-sample, the RMSRE over all VIX options decreases from 0.611 to 0.488 when we add VIX options to the estimation dataset. Such a decrease is observed throughout all maturity and moneyness buckets. The corresponding RMSE goes down from 0.402 to 0.276. Out-of-sample, the numbers are comparable, with a decrease of the RMSRE from 0.555 to 0.475 and a decrease of the RMSE from 0.401 to 0.350 . We note that the out-of-sample pricing errors are smaller than the in-sample errors, which is due to the fact that the financial crisis overlaps to a large part with our in-sample period.

The mispricing of VIX options, when they are not included in the estimation dataset, indicates that the conditional $\mathbb{Q}$-distributions of variance over the time horizons covered by options' maturities are not well represented. A comparison of the gain in RMSREs between the VIX options expiring in less than two months and the others, when including VIX options in the dataset, confirms this result. In-sample (out-of-sample), the gain for short-term options is indeed 0.099 (0.096), versus 0.167 (0.074) for long-term options. Results are unchanged when using the benchmark model. ${ }^{14}$

To assess whether the differences between pricing errors obtained with the two estimation datasets are significant, we run Diebold-Mariano tests. The time- $t$ loss function is given by the mean square relative error between model-implied option prices and observed prices. Denote the loss differential between the errors produced with estimation datasets without and with VIX options by $d_{t}$. Under the null hypothesis that the two estimation datasets produce pricing errors of equal magnitude, $\mathbb{E}\left[d_{t}\right]=0$.

\footnotetext{
${ }^{14}$ We also analyze pricing errors for sub-specifications of the SVJ3 model. They are larger due to the restrictions imposed, but confirm the observations made for the SVJ3 model. In unreported results, we find that the stochastic central tendency significantly improves the pricing of long-term options and the representation of the tails of the distributions of the returns (OTM puts and calls on the S\&P 500). Furthermore, jumps improve the representation of the right tail of the variance distribution (OTM calls on the VIX) as well as of the short-term options. Pricing errors for sub-specifications of the SVJ3 model are available upon request.
} 
If the estimation including VIX options produces smaller pricing errors than the estimation excluding them, then $\mathbb{E}\left[d_{t}\right]>0$. The Diebold-Mariano statistic is the $t$-statistic for this test. Table 7 reports the results. ${ }^{15}$ The Diebold-Mariano tests strongly confirm that VIX options are better priced when included in the estimation dataset. Additional model diagnostics are reported in Appendix G.

[Table 7 about here.]

\section{3. $\quad$ Filtered trajectories}

In Figure 2, we plot the trajectories of the volatility processes $v$ and $m$, filtered using the SVJ3 and the benchmark models. Panel A represents the trajectory of $v$ including VIX options in the estimation dataset. The trajectories for $v$ obtained using the SVJ3 and the benchmark model overlap almost perfectly. Note that $v$ never touches or crosses the zero boundary.

[Fig. 2 about here.]

Panel B represents the filtered trajectory of the stochastic central tendency $m$. The process $m$ is overall more persistent than the process $v$, in line with the parameter estimates found. It starts increasing in mid-2007 from around 1\% to around 5\%. It stabilizes and oscillates around that level for about a year, until September 2008 when it increases gradually again, to reach a level close to $10 \%$ at the beginning of 2009. This increase is followed by a gradual decrease until to a level, in 2016, that is slightly above the initial level of 2006. The high volatility due to the flash crash in May 2010 translates into an slight increase of $m$ from 5 to $7 \%$. While the process $v$ reaches levels which are close to its initial level from the beginning of 2010, $m$ reverts at a much slower pace.

In Panel $\mathrm{C}$, we plot the filtered trajectory of the jump intensity factor $u$. The factor $u$ acts as correction factor in times of market stress and wildly oscillates about its long-term mean $\theta_{u}=1$. The factor $u$ therefore corrects for the non-linearity in $v$ of the intensity of jumps. Panel D represents the recovered jump sizes in $v$, when the estimated probability of jumps is larger than $5 \%$. Note that few negative jumps in the volatility are filtered, the main one occurring at the peak of volatility, hence causing no risk that the volatility level crosses zero.

\footnotetext{
${ }^{15}$ The standard errors are computed using the Newey and West (1987) autocorrelation and heteroscedasticity consistent variance estimator with the number of lags optimally chosen according to Andrews (1991).
} 


\subsection{Representation of the variance term structure}

To further assess the model's ability to reproduce the variance of S\&P 500 returns, we test whether it can price claims on the variance which are not included in the estimation dataset. While the VIX measures the expectation of future variance over a 30-day horizon, it is straightforward to construct a similar index for other maturities, using S\&P 500 options with the chosen maturities. In fact, in 2007 the CBOE started calculating the three-month VXV index and in 2013 the 9-day VXST index. We build indices for maturities of two, three, five, and six months and verify that our model can reproduce them. We calculate the RMSEs for the SVJ3 and the benchmark model. The RMSE on the VIX square during the in-sample period, obtained from daily data, is $2.6 \%$, and goes down to $1.6 \%$ when excluding the data after September 2008 from the calculation. It is equal to $1.8 \%$ in the out-of-sample period. The VIX index is therefore well fitted, whether VIX options are in the estimation dataset or not. Synthesizing a VIX index for other maturities generates a small error, which depends on the amount of traded options for the considered maturities. As there are less traded options for longer time to maturity options, we expect the six-month VIX to be less precisely synthesized than the 30-day VIX index. RMSEs are reported in Table 8.

[Table 8 about here.]

Including VIX options in the estimation dataset, we obtain RMSEs on the synthesized two, three, five, and six-month squared VIX indexes, obtained from weekly data, between $1.4 \%$ and $2.5 \%$ over the entire time period considered (in- and out-of-sample). In contrast, when we exclude VIX options from the estimation dataset, we obtain RMSEs between $1.5 \%$ and $3.1 \%$. These results provide striking evidence that VIX options play a fundamental role in identifying the parameters of the model that drive the variance term structure. The same ordering is noticed for both models examined, namely the SVJ3 and the benchmark model. This result holds particularly true in the in-sample period, which is much more hectic than the out-of-sample period. Excluding the period starting in September 2008, i.e., the market distress period, from the in-sample period, does not change the result. Therefore, VIX options provide information over the whole time period, disregarding market conditions. This holds for both models examined. 


\section{Variance risk premium}

From Section 5 we learn that including VIX options in the estimation dataset allows us to achieve a more precise representation of the risk-neutral conditional distribution of future variance. The variance risk premium (VRP) in Eq. (13) is by construction highly dependent on the dynamics of variance under both historical and risk-neutral measure. In this section, we analyze the dynamic properties of the VRP, using the estimation performed with the full dataset, i.e., we include VIX options.

\subsection{Estimated VRP}

We estimate the VRP for investments with different times to maturity, varying from one week to one year. In Figure 3, Panel A, we plot the evolution of the VRP over time, with its 90\% confidence interval. In line with literature, we find that the VRP is negative. At the beginning of our sample period, it is stable around $-1 \%$, meaning that investors are willing to pay $1 \%$ of their notional per year to be hedged against variance fluctuations, when investing in a six-month contract. From mid-2007, the VRP reacts to the slight increase in volatility, goes down, and stays around $-2 \%$ until Lehman Brothers' bankruptcy. This event triggers a sharp drop to almost $-12 \%$, followed by a recovery period bringing the VRP back to $-2 \%$. The 2010 flash crash prompts a second drop to $-5 \%$, followed by a second recovery period. In contrast with the volatility factor, the VRP never goes back to its initial level of $-1 \%$.

[Fig. 3 about here.]

Panel B of Figure 3 represents the VRP for different times to maturity ranging from one month to one year. The sign and shape of the VRP are consistent across maturities. Before September 2008, the VRP range between 0 and $-2.5 \%$, exhibiting a flat term structure. In September 2008, they all drop simultaneously but then recover at different paces. The shorter-term VRP recover faster than the longer-term VRP.

In Panels $\mathrm{C}$ and $\mathrm{D}$ of Figure 3, we decompose the one-week and six-month VRP into their continuous and discontinuous parts. The discontinuous component of the VRP dominates for shorter maturities, indicating that including jumps helps the representation of the shorter end of the VRP's 
term structure. In fact, the jump part of the VRP constitutes about two thirds of the total VRP for a one-week investment. At the long end, the effect of a jump in the variance process is likely to be dampened by the reversion of the variance to its long-term mean, which justifies why jumps only have a moderate impact on the six-month VRP.

Finally, Panels $\mathrm{E}$ and $\mathrm{F}$ of Figure 3 represent the contributions of the $v$ and $m$ factors to the VRP. The contribution of $m$ to the VRP is negligible for short maturities but plays a substantial role for maturities larger than three months, which explains why the one-week VRP recovers much faster than the six-month VRP after the volatility peak. The central tendency $m$ plays an important role in setting the level of the continuous VRP, especially for mid- to long-term investments, but it becomes secondary during market turmoil. The VRP is then dominated by the impact of $v$. Hence, summarizing the above dicussion, we find that both the variance jumps and the stochastic central tendency play a crucial role in the VRP. While the jumps help represent the short end of the variance term structure, the central tendency has a large impact on the mid- to long-term VRP, especially during market calm. In times of financial crises, the impact of the process $v$ dominates.

[Fig. 4 about here.]

Figure 4 compares the VRP to its model-free ex-ante estimate and to its ex-post approximated value, for the one-month and six-month horizons. The ex-ante estimate is computed as the difference between the past realized variance (over the last 30 days for the one-month VRP and over the last six months for the six-month VRP) and the current value of the VIX index. As the expectation of the future realized variance is not available in a model-free way, this estimate assumes that it can be approximated by the past realized variance. The ex-post approximation is calculated as the difference between the observed realized variance at maturity and the VIX value at the beginning of the observation period. In times of market turmoil, the realized variance is much higher than its expected value, causing the ex-post VRP to be highly positive and to vary in an erratic way around it conditional first moment. 


\subsection{Term structure of the variance risk premium}

While the negativity of VRP is well established, the term structure of VRP has been subject to scrutiny recently. Indeed, in contrast to most papers finding a VRP that is more negative when time to maturity increases (e.g., Amengual (2008)), Gruber, Tebaldi, and Trojani (2015) find instead that the slope of the term structure switches sign in times of market distress. Such phenomenon has already been uncovered for the equity risk premium by van Binsbergen, Hueskes, Koijen, and Vrugt (2013), and is intuitively appealing from an economic perspective. Indeed, in low volatile periods, investors are likely to require a larger compensation for a long-term investment, as the probability that markets enter into distress before expiry increases with time horizon. However, in highly volatile periods and due to the mean-reverting nature of volatility, increasing the time to maturity of an investment also increases the probability that volatility will go down before expiry of the investment. This increase in probability justifies a lower (in absolute value) VRP for long-term investments during of market distress.

Panel A of Figure 5 plots the difference between the three-month and the nine-month VRP over time. This difference is generally positive, meaning that the 9-month VRP is more negative than the three-month VRP. During the recession (shaded area) this relation is inversed, which is in line with the findings of Gruber, Tebaldi, and Trojani (2015), and the difference becomes highly negative. In terms of modeling, we have seen that there is a large premium for jump variance, which represents about two times the premium for continuous variance fluctuations for a one-week investment. This premium disappears when increasing time to maturity of the investment. Panels B and C of Figure 5 represent the continuous and jump contributions in our estimation and show that even though both contributions of the VRP to the three-month minus one-year difference are responsible for the change in sign, the jump component switches more often than the continuous component.

[Fig. 5 about here.]

As the above results could be the artefact of our model specification, we seek to confirm them in a model-free way. The $\mathbb{P}$-leg of the VRP is not observable. However, the $\mathbb{Q}$-leg can be well approximated, up to an adjustment due to jumps, by the VIX index. In Figure 5, Panel D plots the difference between the three-month and the six-month VIX, constructed from S\&P 500 options 
following the procedure used in Section 5.4. It indicates that the slope of the term structure of the $\mathbb{Q}$-expectation of total variance becomes strongly positive during the recession, driving the slope of the term structure of the variance risk premium down. This observation suggests that our results are not driven by model mis-specification.

Another property of the VRP term structure that has been recently investigated in the literature is its convexity (or concavity, in papers which define the VRP as the $\mathbb{Q}$-expectation of variance minus the $\mathbb{P}$-expectation). See, e.g., Andries, Eisenbach, Schmalz, and Wang (2015) and Dew-Becker, Giglio, Le, and Rodriguez (2017). The latter argue that shocks in the future variance (beyond the first months) are not priced, and that only transitory shocks in the short-term variance are priced. This theory is consistent with a term structure of VRP which is steep for short-term maturities and flattens out for longer-term maturities. In Panel A of Figure 6, we plot the term structure of VRP on different dates and show that the level of skewness and convexity of the VRP term structure is timedependent. Before Lehman Brothers filed for bankruptcy, the term structure of VRP is decreasing and flat after the three-month horizon, corroborating the findings of Dew-Becker, Giglio, Le, and Rodriguez (2017). However, in October 2008, the shape of the VRP term structure radically changes. It is no longer flat for long horizons and, moreover, it exhibits a sharp decline up to a horizon of around three months, reaches its minimum and then increases almost as sharply for longer-term investments. The inversion in the term structure of VRP is only happening for mid- to long-term investments. Intuitively, this finding is consistent with the market's belief that the situation could get even worse in the short-term, but will eventually get better in the long-term.

[Fig. 6 about here.]

Panel B of Figure 6 represents an estimate of the higher-order moments of the VRP term structure. The short-term skewness is measured as the difference between the one-month and the threemonth VRP, while the long-term skewness represents the three-month VRP minus the one-year VRP. They hence represent the insurance premium an investor would be willing to pay to be hedged against fluctuations in the forward variance. The convexity is measured as the sum of the one-month and the six-month VRP minus two times the three-month VRP. The graph shows that the short-term skewness of the VRP term structure varies together with its convexity, in line with what is illustrated 
in Panel A. The long-term skewness varies in the inverse direction. In fact, the three time series are almost perfectly correlated with a correlation coefficient above 0.96 .

Our results are complementary to the ones of Gruber, Tebaldi, and Trojani (2015) and DewBecker, Giglio, Le, and Rodriguez (2017). Compared to the former, we only find a switch in the slope of the VRP term structure over mid- to long-term maturities. For short-term horizons, we find that the slope becomes more pronounced in times of market turmoil, causing our convexity indicator to increase. Compared to the latter, we find that investors do care about shocks in future variance, but that the way they care differs across time, depending on economic conditions.

\section{Economic implications}

In this section, we analyze two important applications of our model. First, we seek to use the information contained in the VRP term structure as a trading signal for a volatility strategy. Second, we investigate the predictive power of our VRP estimates on future S\&P 500 returns.

\subsection{Investing in variance}

The switch in the slope of the VRP term structure has strong economic implications. Indeed, it implies that it is no longer profitable, on average, to sell claims on variance with a longer time to maturity and hedge part of the exposure by buying claims with a shorter time to maturity. ${ }^{16}$ We start our investment analysis by asking whether the term structure of VRP can be used as trading signal. In the spirit of Dew-Becker, Giglio, Le, and Rodriguez (2017), we define a squared VIX forward as a claim on the future variance with the following payoff:

$$
\mathrm{VIX}_{t, T_{1}, T_{2}}^{2}=\mathrm{VIX}_{t, T_{2}}^{2}-\mathrm{VIX}_{t, T_{1}}^{2}
$$

Hence, $\mathrm{VIX}_{t, T_{1}, T_{2}}^{2}$ represents the value of a portfolio that is long variance with maturity $T_{2}$ and short variance with maturity $T_{1} \cdot{ }^{17}$ Assuming that there exists an index tracking the squared VIX forward

\footnotetext{
${ }^{16}$ Such investment has been shown to be dynamically optimal in Egloff, Leippold, and Wu (2010) and Filipović, Gourier, and Mancini (2016) using variance swaps.

${ }^{17}$ Dew-Becker, Giglio, Le, and Rodriguez (2017) build these claims for $T_{2}-T_{1}=1$ month. Such payoff can be attained by a long-short strategy in variance swaps with maturities $T_{1}$ and $T_{2}$, or equivalently (up to a jump term) by a portfolio of S\&P 500 options.
} 


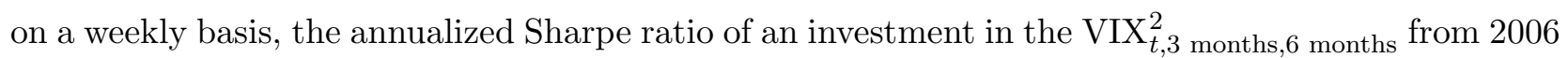
until 2016 is equal to 0.01 . On average, a simple trading strategy that is long forward variance is therefore not profitable for this period, in line with Dew-Becker, Giglio, Le, and Rodriguez (2017). ${ }^{18}$

Taking a closer look at the data and in particular at the slope of the VRP term structure, we find that this meager performance can be explained as follows. As long as realized variance is low, i.e., outside the financial crisis, the strategy yields a positive payoff equal to the difference between the three-month and the six-month VRP. This difference becomes negative during the financial crisis, causing losses for the portfolio holder and canceling out the previous gains.

Therefore, we can interpret a switch in the sign of the slope of the VRP term structure as a warning that the future realized variance may increase, as a result of which the forward variance risk premium is no longer positive. If one leaves the investment on hold until the slope switches sign again, one can avoid some of the losses of the former strategy and generate a Sharpe ratio of 0.46. Switching position from selling future variance into buying future variance whenever the slope of the VRP term structure is negative further enhances the Sharpe ratio, which reaches 0.77.

The returns on forward variance and the times when the strategy is kept on hold are displayed in Figure 7. The investment in forward variance is only interrupted seven weeks during the whole time period, starting in October 2008. The large improvement in the Sharpe ratio is achieved during this short period of time.

\section{[Fig. 7 about here.]}

Using the benchmark model, these results still hold and Sharpe ratios become respectively 0.17 and 0.32. Hence, we can interpret the sign of the VRP slope as a trigger indicating that selling future variance is no longer profitable. These results are robust to other values of $T_{1}$ and $T_{2}$. For example, the previously reported Sharpe ratios become $-0.01,0.10$, and 0.15 respectively if using $T_{1}=$ four months instead of three months, and $0.01,0.21$ and 0.23 if using $T_{2}=9$ months. In the latter case, our algorithm detects an additional switch in August 2015, triggered by the Chinese Yuan devaluation.

\footnotetext{
${ }^{18}$ Dew-Becker, Giglio, Le, and Rodriguez (2017) note that for $T_{2}$ larger than 2 months and $T_{2}-T_{1}=$ a month, Sharpe ratios are "insignificantly different from zero". The disappointing performance for this simple trading strategy is mostly caused by punctual losses occurring during the financial crisis in 2008.
} 


\section{2. $\quad$ Return predictability}

Bollerslev, Tauchen, and Zhou (2009), using the difference between implied and realized variances as a proxy for the VRP, find that it has predictive power on future returns, large premia predicting high future S\&P 500 returns. Their finding is robust and has been confirmed in subsequent papers. ${ }^{19}$ More generally, as we work in a affine setup and the VRP is a linear function of the state variables, we investigate the predictive power of the three state variables in our model on S\&P 500 returns.

Our results are summarized in Table 9, which reports the estimated parameters for all regressions, using monthly observations. ${ }^{20}$ As we see from Table 9, the beta of the VRP is negative, confirming the usual results: the larger the VRP (in absolute value), the larger future returns on average. In contrast, the beta of the term structure variable is always positive, indicating that a larger short-term skewness goes together with higher future returns on average. We also include the difference between the VIX and the (past) one-month realized variance as predictor as in Bollerslev, Tauchen, and Zhou (2009). As expected, the coefficient for this predictor is negative. The coefficient in front of the short-term variance $v$ is positive. Intuitively, the VRP loads heavily on the $v$ factor, with negative loading. The negative coefficient in front of the VRP therefore translates into a positive coefficient in front of the $v$ process. The coefficient in front of the long-term variance process $m$ is negative, indicating that on average, the larger the stochastic central tendency, the smaller the future returns. The coefficient in front of the negative jump intensity process $u$ changes with maturity. It is positive for the one-month horizon, with a $t$-statistics of 1.584. For longer horizons, it loses significance, confirming the intuition that jumps only impact returns on the short-term.

Similarly to Bollerslev, Tauchen, and Zhou (2009), the $R^{2}$ increases with time to maturity, to reach its maximum around four months, and then goes down. This pattern holds for all regressions. The main difference between using the model-free estimator and the model-implied VRP estimates comes from the rate at which the $R^{2}$ declines after reaching its optimum. For a 5-month maturity, the model-free VRP exhibits an adjusted $R^{2}$ of 0.151 against 0.160 for the model predictors, but for a 7 -month horizon the gap increases: 0.118 versus 0.147 , for 8 months these numbers become 0.09 versus 0.123 . Although the difference is relatively small, this gap suggests that adding model-implied

\footnotetext{
${ }^{19}$ See, among others, Drechsler and Yaron (2011), Bekaert and Hoerova (2014), Kelly and Jiang (2014), and Bali and Zhou (2016).

${ }^{20}$ Our results should be interpreted with caution due to the overlapping windows for horizons larger than a month.
} 
predictors adds value to the predictions. This result holds for both the benchmark model and the SVJ3 model.

Finally, this exercise highlights the information contents of the slope of the VRP term structure. Indeed, running the regression only with the past RV and the VRP yields an adjusted $R^{2}$ that is substantially lower than the one obtained when adding the slope of the VRP term structure, for all horizons considered. This result can be explained in terms of projections on the latent factors. The VRP, in our models, is explained by two to three latent factors, depending on whether we use the benchmark or the SVJ3 model. The slope of the VRP term structure is described by these factors as well. Adding it to the regression therefore allows spanning the information available in the two dominant factors $v$ and $m$. For the benchmark model, the spanning is perfect in the sense that the VRP and the slope of its term structure are perfectly described by a weighted average of $v$ and $m$. For the SVJ3 model, there is a slight loss of information. This loss of information is quantified by the differences between the second and the fourth regressions, i.e., the regression using the VRP and its term structure and the one using all latent factors $v, m$ and $u$. The resulting adjusted $R^{2}$ are fairly similar, the largest difference being attained for a three-month horizon: 0.167 versus 0.174 .

Our results therefore show that similarly to the variance which is described by two to three factors, the VRP contains more than one dimension of interest, including its level but also the slope of its term structure. The latter dimension matters not only for its implications in terms of trading strategy, but also for its valuable information contents on future returns.

[Table 9 about here.]

\section{Conclusion}

In this paper, we carry out an extensive empirical investigation of the information contained in VIX options on the dynamics of S\&P 500 returns and their variance process. We estimate various specifications of a flexible affine model using two datasets. The first dataset contains a time series of S\&P 500 and VIX indexes as well as S\&P option prices, and the second dataset contains VIX options in addition to the first dataset. We do not restrict the moneyness and maturity of the options considered, so that we can fully benefit from their information contents on the distribution 
of S\&P 500 returns and their variance. Instead of a step-wise estimation, we depart from most of the literature and estimated the historical and the risk-neutral parameters jointly, in a single step. Our maximum likelihood estimation procedure is based on particle filtering.

We find that VIX options contain information on the dynamics properties of S\&P 500 returns, which is not spanned by S\&P 500 options. This conclusion is based on several observations. First, when VIX options are not included in the estimation dataset, they are not well priced. Including them in the estimation dataset not only improves the pricing of VIX options in both in- and outof-sample periods, but also the pricing of S\&P 500 options. In-sample, deep OTM put options and long-term options are better priced and so are all put options out-of-sample. Second, including VIX options in the estimation dataset allows considerable improvements in representing the variance term structure. We replicate the construction of the VIX index for maturities two, three, five, and six months. We find that for maturities larger than three months, the model yields RMSEs which are significantly larger when VIX options are excluded from the dataset.

We perform a thorough analysis of the variance risk premium (VRP) and of its term structure. In line with the literature, we find that the VRP, in absolute value, is more negative for long-term investments in low volatility periods. However, during high volatility periods, we uncover a non-monotonic term structure of VRP, which reaches its maximum around a three-month maturity. This finding complements the recent results of Gruber, Tebaldi, and Trojani (2015) and Dew-Becker, Giglio, Le, and Rodriguez (2017). We illustrate the economic implications of our results by testing a simple trading strategy, which sells long-maturity variance and buys short-maturity variance, switching the sign of positions when an inversion of the term structure occurs. Our strategy allows reaping the variance risk premium when conditioning on market conditions. It generates a Sharpe ratio of 0.77 , compared to a meager ratio of 0.01 for a strategy that does not react to changes in the VRP term structure. Finally, we study the predictive power of our model-implied VRP and its term structure on future S\&P 500 returns. We find that adding a term structure component substantially improves the predictive power of the VRP and explain our results in terms of spanning of the latent factors that describe variance. 


\section{References}

Aït-Sahalia, Y., Karaman, M., Mancini, L., 2012. The term structure of variance swaps, risk premia and the expectation hypothesis. Working paper. Princeton University and Swiss Finance Institute.

Aït-Sahalia, Y., Kimmel, R., 2007. Maximum likelihood estimation of stochastic volatility models. Journal of Financial Economics 83, 413-452.

Aït-Sahalia, Y., Lo, A., 1998. Nonparametric Estimation of State-Price Densities Implicit in Financial Asset Prices. Journal of Finance 53, 499-547.

Alizadeh, S., Brandt, M. W., Diebold, F. X., 2002. Range-based estimation of stochastic volatility models. Journal of Finance 57, 1047-1091.

Amengual, D., 2008. The term structure of variance risk premia. Working Paper.

Amengual, D., Xiu, D., 2015. Resolution of policy uncertainty and sudden declines in volatility. Chicago Booth Paper No. 13-78.

Andersen, T., Fusari, N., Todorov, V., 2015. The risk premia embedded in index options. Journal of Financial Economics Forthcoming.

Andersen, T. G., Benzoni, L., Lund, J., 2002. An empirical investigation of continuous-time equity return models. Journal of Finance 57, 1239-1284.

Andersen, T. G., Bollerslev, T., Diebold, F. X., Ebens, H., 2001. The distribution of realized stock return volatility. Journal of Financial Economics 61, 43-76.

Andries, M., Eisenbach, T., Schmalz, M., Wang, Y., 2015. The term structure of the price of variance risk. Federal Reserve Bank of New York Staff Report No. 736.

Bakshi, G., Cao, C., Chen, Z., 1997. Empirical performance of alternative option pricing models. Journal of Finance 52, 2003-2049.

Bali, T. G., Zhou, H., 2016. Risk, uncertainty and expected returns. Journal of Financial and Quantitative Analysis 51, 707-735. 
Barndorff-Nielsen, O. E., Shephard, N., 2004. Power and bipower variation with stochastic volatility and jumps. Journal of Financial Econometrics 2, 1-48.

Bates, D. S., 1996. Jumps and stochastic volatility: Exchange rate processes implicit in Deutsche Mark options. Review of Financial Studies 9, 69-107.

Bates, D. S., 2000a. Post-87 crash fears in the S\&P 500 futures option market. Journal of Econometrics $94,181-238$.

Bates, D. S., 2000b. Post-87 crash fears in the S\&P 500 futures options. Journal of Econometrics 94, $181-238$.

Bates, D. S., 2006. Maximum likelihood estimation of latent affine processes. The Review of Financial Studies 19, 909-965.

Bates, D. S., 2012. U.S. stock market crash risk, 1926-2010. Journal of Financial Economics 105, 229 $-259$.

Bayer, C., Gatheral, J., Karlsmark, M., 2013. Fast Ninomiya-Victoir calibration of the double-meanreverting model. Quantitative Finance 13, 1813-1829.

Bekaert, G., Hoerova, M., 2014. The vix, the variance premium and stock market volatility. Journal of Econometrics 183, 181-192.

Bollerslev, T., Tauchen, G., Zhou, H., 2009. Expected stock returns and variance risk premia. Review of Financial Studies 22, 4463-92.

Bollerslev, T., Todorov, V., 2011. Tails, fears and risk premia. Journal of Finance 66, 2165-2211.

Branger, N., Kraftschik, A., Völkert, C., 2016. The fine structure of variance: Pricing vix derivatives in consistent and log-vix models. Working Paper.

Broadie, M., Chernov, M., Johannes, M., 2007. Model specification and risk premia: Evidence from futures options. Journal of Finance 62, 1453-1490.

Broadie, M., Chernov, M., Johannes, M., 2009. Understanding index option returns. The Review of Financial Studies 22, 4493-4529. 
Carr, P., Madan, D. B., 1999. Option valuation using the fast fourier transform. Journal of Computational Finance 2, 1-18.

Chernov, M., Gallant, A. R., Ghysels, E., Tauchen, G. T., 2003. Alternative models of stock price dynamics. Journal of Econometrics 116, 225 - 257.

Christoffersen, P., Heston, S., Jacobs, K., 2009. The shape and term structure of the index option smirk: Why multifactor stochastic volatility models work so well. Management Science 55, 19141932.

Christoffersen, P., Jacobs, K., Mimouni, K., 2010. Models for S\&P 500 dynamics: Evidence from realized volatility, daily returns and options prices. Review of Financial Studies 23, 3141-3189.

Chung, S.-L., Tsai, W.-C., Wang, Y.-H., Weng, P.-S., 2011. The information content of the S\&P 500 index and VIX options on the dynamics of the S\&P 500 index. The Journal of Futures Markets $31,1170-1201$.

Cont, R., Kokholm, T., 2013. A consistent pricing model for index options and volatility derivatives. Mathematical Finance 23, 248-274.

Dew-Becker, I., Giglio, S., Le, A., Rodriguez, M., 2017. The price of variance risk. Journal of Financial Economics 123.

Drechsler, I., Yaron, A., 2011. What's vol got to do with it?. Review of Financial Studies 24, 1-45.

Duan, J.-C., Yeh, C.-Y., 2010. Jump and volatility risk premiums implied by vix. Journal of Economic Dynamics and Control 34, 2232-2244.

Duan, J.-C., Yeh, C.-Y., 2011. Price and volatility dynamics implied by the vix term structure. Working paper. NUS RMI Working Paper No. 11/05.

Duffie, D., Pan, J., Singleton, K. J., 2000. Transform analysis and asset pricing for affine jumpdiffusions. Econometrica 68, 1343-1376.

Durham, G. B., 2013. Risk-neutral modeling with affine and nonaffine models. Journal of Financial Econometrics 11, 650-681. 
Egloff, D., Leippold, M., Wu, L., 2010. Valuation and optimal investing in variance swaps. Journal of Financial and Quantitative Analysis 45, 1279-1310.

Eraker, B., 2004. Do stock prices and volatility jump? Reconciling evidence from spot and option prices. Journal of Finance 59, 1367-1404.

Eraker, B., Johannes, M. S., Polson, N., 2003. The impact of jumps in equity index volatility and returns. Journal of Finance 58, 1269-1300.

Fang, F., Oosterlee, C. W., 2008. A novel pricing method for European options based on Fouriercosine series expansions. SIAM Journal on Scientific Computing 31, 826-848.

Ferriani, F., Pastorello, S., 2012. Estimating and testing non-affine option pricing models with a large unbalanced panel of options. The Econometrics Journal 15, 171-203.

Filipović, D., Gourier, E., Mancini, L., 2016. Quadratic variance swap models. Journal of Financial Economics 119, 44-68.

Gatheral, J., 2008. Consistent modeling of spx and vix options. The Fifth World Congress of the Bachelier Finance Society, London, July 2008.

Gordon, N. J., Salmond, D. J., Smith, A. F. M., 1993. Novel approach to nonlinear/non-Gaussian Bayesian state estimation. Radar and Signal Processing, IEE Proceedings F 140, 107-113.

Gruber, P. H., Tebaldi, C., Trojani, F., 2015. The price of the smile and variance risk premia. Swiss Finance Institute Research Paper No. 15-36.

Hansen, N., Ostermeier, A., 1996. Adapting arbitrary normal mutation distributions in evolution strategies: The covariance matrix adaptation. Proceedings of the 1996 IEEE Conference on Evolutionary Computation (ICEC 96) pp. 312-317.

Hu, F., Zidek, J. V., 2002. The weighted likelihood. The Canadian Journal of Statistics 30, 347-371.

Huang, D., Shaliastovich, I., 2015. Volatility-of-volatility risk. Working Paper, Wharton School, University of Pennsylvania. 
Jacod, J., Todorov, V., 2010. Do price and volatility jump together?. Annals of Applied Probability 20, $1425-1469$.

Jiang, G. J., Tian, Y. S., 2007. Extracting model-free volatility from option prices: An examination of the VIX index. The Journal of Derivatives 14, 35-60.

Johannes, M. S., Polson, N. G., Stroud, J. R., 2009. Optimal filtering of jump diffusions: Extracting latent states from asset prices. Review of Financial Studies 22, 2759-2799.

Johnson, T. L., 2012. Equity risk premia and the vix term structure. Working paper, Stanford University.

Jones, C. S., 2003. The dynamics of stochastic volatility: Evidence from underlying and options markets. Journal of Econometrics 116, 181-224.

Kaeck, A., Alexander, C., 2012. Volatility dynamics for the s\&p 500: Further evidence from nonaffine, multi-factor jump diffusions. Journal of Banking \& Finance 36, 3110 - 3121.

Kelly, B., Jiang, H., 2014. Tail risk and asset prices. Review of Financial Studies 27, 2841-2871.

Kloeden, P. E., Platen, E., 1992. Numerical Solution of Stochastic Differential Equations. SpringerVerlag, Berlin, Germany.

Lindström, E., Ströjby, J., Brodén, M., Wiktorsson, M., Holst, J., 2008. Sequential calibration of options. Computational Statistics \& Data Analysis 52, 2877-2891.

Mencía, J., Sentana, E., 2013. Valuation of vix derivatives. Journal of Financial Economics 108, 367 $-391$.

Ornthanalai, C., 2014. Lévy jump risk: Evidence from options and returns. Journal of Financial Economics 112, 69-90.

Pan, J., 2002. The jump-risk premia implicit in options: Evidence from an integrated time-series study. Journal of Financial Economics 63, 3-50.

Papanicolaou, A., Sircar, R., 2014. A regime-switching heston model for vix and s\&p 500 implied volatilities. Quantitative Finance 14, 1811-1827. 
Pitt, M. K., 2002. Smooth particle filters for likelihood evaluation and maximisation. Working paper 651 The Warwick Economics Research Paper Series.

Pitt, M. K., Shephard, N., 1999. Filtering via simulation: Auxiliary Particle Filters. Journal of the American Statistical Association 94, 590-599.

Rebonato, R., Cardoso, T., 2004. Unconstrained fitting of implied volatility surfaces using a mixture of normals. Journal of Risk 7, 55-74.

Sepp, A., 2008a. Pricing options on realized variance in the heston model with jumps in returns and volatility. Journal of Computational Finance 11, 33-70.

Sepp, A., 2008b. VIX option pricing in a jump-diffusion model. Risk Magazine pp. 84-89.

Song, Z., Xiu, D., 2016. A tale of two option markets: Pricing kernels and volatility risk. Journal of Econometrics 190, $176-196$.

Storn, R., 1996. On the usage of differential evolution for function optimization. In: Biennial Conference of the North American Fuzzy Information Processing Society (NAFIPS) pp. 519-523.

Todorov, V., 2010. Variance risk premium dynamics: The role of jumps. Review of Financial Studies $23,345-383$.

Todorov, V., Tauchen, G., 2011. Volatility jumps. Journal of Business and Economic Statistics 29, $356-371$.

van Binsbergen, J. H., Hueskes, W., Koijen, R. S. J., Vrugt, E. B., 2013. Equity yields. Journal of Financial Economics 110, 503-519.

Wu, L., 2011. Variance dynamics: Joint evidence from options and high-frequency returns. Journal of Econometrics 160, 280-287. 


\section{Appendix}

\section{A. Affine dependence of the $\operatorname{VIX}^{2}$ on $v_{t}, m_{t}$ and $u_{t}$}

The expressions for the coefficients $\alpha_{\mathrm{VIX}}, \beta_{\mathrm{VIX}^{2}}, \gamma_{\mathrm{VIX}}$, and $\delta_{\mathrm{VIX}}$ in Proposition 2.1 are given by

$$
\begin{aligned}
\alpha_{\mathrm{VIX}}= & \left(1+2 \lambda_{1}^{(+)} C^{(+)}+2 \lambda_{1}^{(-)} C^{(-)}\right) A\left(\tau_{\mathrm{VIX}}\right), \\
\beta_{\mathrm{VIX}^{2}=} & \left(1+2 \lambda_{1}^{(+)} C^{(+)}+2 \lambda_{1}^{(-)} C^{(-)}\right) B\left(\tau_{\mathrm{VIX}}\right)+\left(2 \lambda_{2}^{(+)} C^{(+)}+2 \lambda_{2}^{(-)} C^{(-)}\right) \hat{A}\left(\tau_{\mathrm{VIX}}\right), \\
\gamma_{\mathrm{VIX}}= & \left(1+2 \lambda_{1}^{(+)} C^{(+)}+2 \lambda_{1}^{(-)} C^{(-)}\right) D\left(\tau_{\mathrm{VIX}}\right)+\left(2 \lambda_{3}^{(+)} C^{(+)}+2 \lambda_{3}^{(-)} C^{(-)}\right) \bar{A}\left(\tau_{\mathrm{VIX}}\right), \\
\delta_{\mathrm{VIX}}= & 2\left(\lambda_{0}^{(+)} C^{(+)}+\lambda_{0}^{(-)} C^{(-)}\right)+\left(1+2 \lambda_{1}^{(+)} C^{(+)}+2 \lambda_{1}^{(-)} C^{(-)}\right) G+ \\
& \left(2 \lambda_{2}^{(+)} C^{(+)}+2 \lambda_{2}^{(-)} C^{(-)}\right) \hat{B}\left(\tau_{\mathrm{VIX}}\right)+\left(2 \lambda_{3}^{(+)} C^{(+)}+2 \lambda_{3}^{(-)} C^{(-)}\right) \bar{B}\left(\tau_{\mathrm{VIX}}\right),
\end{aligned}
$$

where $C^{(+/-)}:=\frac{1}{1-\mu_{Y}^{(+/-)}}, \tau_{\mathrm{VIX}}$ is 30 days, and the remaining coefficients are available in closed form from:

$$
\begin{array}{ll}
A(\tau)=\frac{1}{\tau_{\mathrm{VIX}}} \int_{t}^{t+\tau_{\mathrm{VIX}}} \alpha_{v}(t, s) d s, & B(\tau)=\frac{1}{\tau_{\mathrm{VIX}}} \int_{t}^{t+\tau_{\mathrm{VIX}}} \beta_{v}(t, s) d s, \\
D(\tau)=\frac{1}{\tau_{\mathrm{VIX}}} \int_{t}^{t+\tau_{\mathrm{VIX}}} \gamma_{v}(t, s) d s, & G(\tau)=\frac{1}{\tau_{\mathrm{VIX}}} \int_{t}^{t+\tau_{\mathrm{VIX}}} \delta_{v}(t, s) d s, \\
\hat{A}(\tau)=\frac{1}{\tau_{\mathrm{VIX}}} \int_{t}^{t+\tau_{\mathrm{VIX}}} \alpha_{m}(t, s) d s, & \hat{B}(\tau)=\frac{1}{\tau_{\mathrm{VIX}}} \int_{t}^{t+\tau_{\mathrm{VIX}}} \beta_{m}(t, s) d s, \\
\bar{A}(\tau)=\frac{1}{\tau_{\mathrm{VIX}}} \int_{t}^{t+\tau_{\mathrm{VIX}}} \alpha_{u}(t, s) d s, & \bar{B}(\tau)=\frac{1}{\tau_{\mathrm{VIX}}} \int_{t}^{t+\tau_{\mathrm{VIX}}} \beta_{u}(t, s) d s .
\end{array}
$$

The functions $\alpha_{v}, \beta_{v}, \gamma_{v}, \delta_{v}, \alpha_{m}, \beta_{m}, \alpha_{u}$, and $\beta_{u}$ are also available in closed-form from:

$$
\begin{aligned}
\mathbb{E}_{t}^{\mathbb{Q}}\left[v_{s}\right] & =\alpha_{v}(t, s) v_{t}+\beta_{v}(t, s) m_{t}+\gamma_{v}(t, s) u_{t}+\delta_{v}(t, s), \\
\mathbb{E}_{t}^{\mathbb{Q}}\left[m_{s}\right] & =\alpha_{m}(t, s) m_{t}+\beta_{m}(t, s), \\
\mathbb{E}_{t}^{\mathbb{Q}}\left[u_{s}\right] & =\alpha_{u}(t, s) u_{t}+\beta_{u}(t, s),
\end{aligned}
$$

for $0 \leq t<s$. 


\section{B. Characteristic functions}

The characteristic functions of the processes $Y, \mathrm{VIX}^{2}$ are exponential affine in the state processes:

$$
\begin{aligned}
& \Psi_{V I X_{T}^{2}}(t, v, m, u ; \omega)=\mathbb{E}_{t}^{\mathbb{Q}}\left[e^{\omega V I X_{T}^{2}}\right]=e^{\alpha(T-t)+\beta(T-t) v+\gamma(T-t) m+\delta(T-t) u}, \\
& \Psi_{Y_{T}}(t, v, m, u ; \omega)=\mathbb{E}_{t}^{\mathbb{Q}}\left[e^{\omega Y_{T}}\right]=e^{\alpha_{Y}(T-t)+\beta_{Y}(T-t) y+\gamma_{Y}(T-t) v+\delta_{Y}(T-t) m+\xi_{Y}(T-t) u},
\end{aligned}
$$

where $\omega \in \mathbb{C}$, the coefficients in the definition of $\Psi_{V I X_{T}^{2}}$ satisfy the following ODEs, with $\tau=T-t$ :

$$
\begin{aligned}
& -\alpha^{\prime}(\tau)+\gamma(\tau) \kappa_{m} \theta_{m}+\delta(\tau) \kappa_{u} \theta_{u}+\lambda_{0}^{(-)}\left(\frac{1}{1-\beta(\tau) \nu_{v}^{(+)}}-1\right)+\lambda_{0}^{(+)}\left(\frac{1}{1-\beta(\tau) \nu_{v}^{(-)}}-1\right)=0 \\
& -\beta^{\prime}(\tau)-\beta(\tau) \kappa_{v}+\frac{1}{2} \sigma_{v}^{2} \beta^{2}(\tau)+\lambda_{1}^{(-)}\left(\frac{1}{1-\beta(\tau) \nu_{v}^{(+)}}-1\right)+\lambda_{1}^{(+)}\left(\frac{1}{1-\beta(\tau) \nu_{v}^{(-)}}-1\right)=0 \\
& -\gamma^{\prime}(\tau)-\gamma(\tau) \kappa_{m}+\frac{1}{2} \sigma_{m}^{2} \gamma^{2}(\tau)+\kappa_{v} \beta(\tau)+\lambda_{2}^{(-)}\left(\frac{1}{1-\beta(\tau) \nu_{v}^{(+)}}-1\right)+\lambda_{2}^{(+)}\left(\frac{1}{1-\beta(\tau) \nu_{v}^{(-)}}-1\right)=0 \\
& -\delta^{\prime}(\tau)-\delta(\tau) \kappa_{u}+\frac{1}{2} \delta^{2}(\tau) \sigma_{u}^{2}+\lambda_{3}^{(-)}\left(\frac{1}{1-\beta(\tau) \nu_{v}^{(+)}}-1\right)+\lambda_{3}^{(+)}\left(\frac{1}{1-\beta(\tau) \nu_{v}^{(-)}}-1\right)=0
\end{aligned}
$$

$\forall t \in(0, T]$, with boundary conditions $\alpha(0)=\omega \delta_{V I X^{2}}, \beta(0)=\omega \alpha_{V I X^{2}}, \gamma(0)=\omega \beta_{V I X^{2}}$, and $\delta(0)=$ $\omega \gamma_{V I X^{2}}$, where the coefficients $\alpha_{V I X^{2}}, \beta_{V I X^{2}}, \gamma_{V I X^{2}}$, and $\delta_{V I X^{2}}$ are defined in Appendix A. 
The coefficients of $\Psi_{Y_{T}}$ satisfy the following ODEs for $t \in(0, T]$ :

$$
\begin{aligned}
& -\alpha_{Y}^{\prime}(\tau)+\beta_{Y}(\tau)\left(-\lambda_{0}^{(+)}\left(\frac{1}{1-\mu_{Y}^{(+)}}-1\right)-\lambda_{0}^{(-)}\left(\frac{1}{1-\mu_{Y}^{(-)}}-1\right)\right)+\delta_{Y}(T-t) \kappa_{m} \theta_{m}+\xi_{Y}(\tau) \kappa_{u} \theta_{u}+ \\
& \lambda_{0}^{(+)}\left(\frac{1}{\left(1-\beta_{Y}(\tau) \mu_{Y}^{(+)}\right)\left(1-\gamma_{Y}(\tau) \nu_{v}^{(-)}\right)}-1\right)+\lambda_{0}^{(-)}\left(\frac{1}{\left(1-\beta_{Y}(\tau) \mu_{Y}^{(-)}\right)\left(1-\gamma_{Y}(\tau) \nu_{v}^{(+)}\right)}-1\right)=0, \\
& -\beta_{Y}^{\prime}(\tau)=0, \\
& -\gamma_{Y}^{\prime}(\tau)+\beta_{Y}(\tau)\left(-\lambda_{1}^{(+)}\left(\frac{1}{1-\mu_{Y}^{(+)}}-1\right)-\lambda_{1}^{(-)}\left(\frac{1}{1-\mu_{Y}^{(-)}}-1\right)-\frac{1}{2}\right)-\gamma_{Y}(\tau) \kappa_{v}+\frac{1}{2} \beta_{Y}(\tau)^{2}+ \\
& \frac{1}{2} \gamma_{Y}(\tau)^{2} \sigma_{v}^{2}+\beta_{Y}(\tau) \gamma_{Y}(\tau) \sigma_{v} \rho_{Y, v}+\lambda_{1}^{(+)}\left(\frac{1}{\left(1-\beta_{Y}(\tau) \mu_{Y}^{(+)}\right)\left(1-\gamma_{Y}(\tau) \nu_{v}^{(-)}\right)}-1\right)+ \\
& \lambda_{1}^{(-)}\left(\frac{1}{\left(1-\beta_{Y}(\tau) \mu_{Y}^{(-)}\right)\left(1-\gamma_{Y}(\tau) \nu_{v}^{(+)}\right)}-1\right)=0 \\
& -\delta_{Y}^{\prime}(\tau)+\beta_{Y}(\tau)\left(-\lambda_{2}^{(+)}\left(\frac{1}{1-\mu_{Y}^{(+)}}-1\right)-\lambda_{2}^{(-)}\left(\frac{1}{1-\mu_{Y}^{(-)}}-1\right)\right)+\gamma_{Y}(\tau) \kappa_{v}-\delta_{Y}(\tau) \kappa_{m}+\frac{1}{2} \delta_{Y}(\tau)^{2} \sigma_{m}^{2}+ \\
& \lambda_{2}^{(+)}\left(\frac{1}{\left(1-\beta_{Y}(\tau) \mu_{Y}^{(+)}\right)\left(1-\gamma_{Y}(\tau) \nu_{v}^{(-)}\right)}-1\right)+\lambda_{2}^{(-)}\left(\frac{1}{\left(1-\beta_{Y}(\tau) \mu_{Y}^{(-)}\right)\left(1-\gamma_{Y}(\tau) \nu_{v}^{(+)}\right)}-1\right)=0 \text {, } \\
& -\xi_{Y}^{\prime}(\tau)+\beta_{Y}(\tau)\left(-\lambda_{3}^{(+)}\left(\frac{1}{1-\mu_{Y}^{(+)}}-1\right)-\lambda_{3}^{(-)}\left(\frac{1}{1-\mu_{Y}^{(-)}}-1\right)\right)-\xi_{Y}(\tau) \kappa_{u}+\frac{1}{2} \xi_{Y}(T-t)^{2} \sigma_{u}^{2}+ \\
& \lambda_{3}^{(+)}\left(\frac{1}{\left(1-\beta_{Y}(\tau) \mu_{Y}^{(+)}\right)\left(1-\gamma_{Y}(\tau) \nu_{v}^{(-)}\right)}-1\right)+\lambda_{3}^{(-)}\left(\frac{1}{\left(1-\beta_{Y}(\tau) \mu_{Y}^{(-)}\right)\left(1-\gamma_{Y}(\tau) \nu_{v}^{(+)}\right)}-1\right)=0 \text {, }
\end{aligned}
$$

with boundary conditions $\alpha_{Y}(0)=0, \beta_{Y}(0)=\omega, \gamma_{Y}(0)=0, \delta_{Y}(0)=0$, and $\xi_{Y}(0)=0$. The ODEs can be solved numerically using standard methods. 


\section{Coefficients for the Fourier cosine expansion}

Here we give the expression for $U_{n}^{\mathrm{VIX}}{ }^{2}$, the Fourier cosine transform of the VIX options' payoff. To ease notation, we drop the subscript vIx for $a_{\mathrm{VIX}}, b_{\mathrm{VIX}}$ and define $\omega_{n}:=\frac{n \pi}{b-a}$. For $n>0$, we obtain

$$
\begin{aligned}
U_{n}^{\mathrm{VIX}} & =\int_{a}^{b}\left(\sqrt{x}-K_{\mathrm{VIX}}\right)^{+} \cos \left(\omega_{n}(x-a)\right) d x \\
& =\frac{2}{b-a} \operatorname{Re}\left\{e^{-i \omega_{n} a}\left[\frac{\sqrt{b} e^{-i \omega_{n} b}}{i \omega_{n}}+\frac{\sqrt{\pi}}{2\left(-i \omega_{n}\right)^{3 / 2}}\left(\operatorname{erfz}\left(\sqrt{-i \omega_{n} b}\right)-\operatorname{erfz}\left(K \sqrt{-i \omega_{n}}\right)\right)\right]\right\},
\end{aligned}
$$

where $\operatorname{erfz}(\cdot)$ is the error function for complex numbers. For $n=0$,

$$
U_{0}^{\mathrm{VIX}{ }^{2}}=\frac{2}{b-a}\left[\frac{2}{3} b^{3 / 2}-K_{\mathrm{VIX}} b+\frac{1}{3} K_{\mathrm{VIX}}^{3}\right]
$$

\section{Joint calibration}

In this section, we describe the joint calibration exercise performed using the cross-section of S\&P 500 and VIX options on specific dates. This exercise gives us some guidance for model design and allows us to reduce the set of models to be estimated on a time series of options' data.

Specifically, we fix a date $t$ and consider $\left\{I V_{\mathrm{SPX}, i}^{M k t}\right\}_{i=1 \cdots N_{\mathrm{SPX}}}$, the set of $N_{\mathrm{SPX}}$ market implied volatilities of S\&P 500 options for strikes $\left\{K_{i}\right\}$ and maturities $\left\{T_{i}\right\}$. We denote by $\left\{I V_{\mathrm{VIX}, j}^{M k t}\right\}_{j=1 \cdots N_{\mathrm{VIX}}}$ the set of $N_{\text {VIX }}$ market implied volatilities of VIX options. To estimate the parameters, we minimize the root mean squared error (RMSE) between the market and model implied volatilities: ${ }^{21}$

If a model is not flexible enough to jointly reproduce the implied volatility patterns of both markets on a single date, the $\mathbb{Q}$-dynamics of the model is not sufficiently rich to accurately price both the S\&P 500 and VIX derivatives jointly, and we can safely discard this model from further consideration. We consider two sub-specifications of our full model (SVJ3): (i) $m$ and $u$ are constant (SVJ), (ii) $m$ is stochastic but $u$ is constant (SVJ2). In the full SVJ3 specification, we impose $\theta_{u}$ to 1 and $\lambda_{0}^{(-)}=0$ to improve identification.

[Fig. D.1 about here.]

\footnotetext{
${ }^{21}$ Alternatively, we checked that using distances taking into account the bid-ask spread of IVs as in Cont and Kokholm (2013) does not significantly change the quality of fit. Instead of the RMSE, we also looked at average relative errors (ARE). However, this does not affect our conclusions. The results using ARE are available upon request.
} 
We use two global optimizers to cope with the non-convexity of the calibration problem and the potential existence of multiple local minima, namely the Covariance Matrix Adaptation Evolution Strategy (CMA-ES), introduced by Hansen and Ostermeier (1996), and the Differential Evolution (DE) algorithm introduced by Storn (1996).

For our calibration, we choose a date on which the markets were under stress, namely May 5, 2010 at the beginning of the European sovereign debt crisis. After cleaning our data as described previously, we have 91 VIX options at six different maturities (from 0.04 to 0.46 years) and 486 S\&P 500 options at eleven different maturities (from 0.05 to 0.91 years) available. We emphasize that we perform a joint calibration. Hence, all this data is entered as input to minimize the total RMSE in Eq. (D.2) from the VIX and the S\&P 500 market simultaneously across all available maturities and moneyness.

In Figure D.1, we plot the market and model implied volatilities for the S\&P 500 (Panels A, C, E) and the VIX (Panels B, D, F) for two maturity slices each. For the S\&P 500 options, we choose the two maturities $T=0.05$ and $T=0.3$, and for the VIX options, $T=0.04$ and $T=0.36$.

$$
\begin{aligned}
\operatorname{RMSE}_{\mathcal{M}}(t) & :=\sqrt{\frac{1}{N_{\mathcal{M}}} \sum_{1 \leq i \leq N_{\mathcal{M}}}\left(I V_{\mathcal{M}, i}^{M k t}-I V_{\mathcal{M}, i}^{M o d}\right)^{2}}, \quad \mathcal{M} \in\{\mathrm{SPX}, \mathrm{VIX}\} \\
\operatorname{RMSE}(t) & :=\frac{1}{2}\left(\operatorname{RMSE}_{\mathrm{SPX}}(t)+\operatorname{RMSE}_{\mathrm{VIX}}(t)\right)
\end{aligned}
$$

From Panel A, Figure D.1, we observe that the Heston model provides reasonable results for the S\&P 500 market. However, for the VIX market (Panel B), the Heston model clearly fails to reproduce one of the stylized facts of VIX option markets, namely the positive skew of the implied volatility surface. This failure is most pronounced for the short-term options, where the Heston model generates a significant negative skew. The results for the SVJ model look much more promising. Just by adding jump components to the returns and volatility process, we can now generate the positive skew in the VIX market (Panel D), while providing an almost perfect fit for the S\&P 500 options market. The SVJ model only struggles at the short end of the VIX implied volatility surface. This shortcoming disappears when we extend the SVJ specification to the SVJ2 model by adding the factor $m$. Doing so gives us not only a remarkable fit for the S\&P 500, but also for the VIX 
options market (Panel F). Looking at the RMSEs of the SVJ and SVJ2 models, we find that the SVJ provides an $\mathrm{RMSE}_{\mathrm{SPX}}$ of $1.27 \%$ and an $\mathrm{RMSE}_{\mathrm{VIX}}$ of $11.60 \%$. The SVJ2 model yields $1.17 \%$ and $5.15 \%$, respectively. Hence, while the two models are comparable in terms of their performance on the S\&P 500 options market, there is an obvious difference in the VIX market on the chosen date.

In unreported results, we perform calibration exercises on other days, also including calm periods. Irrespective of the day, we observe that the SVJ and SVJ2 models perform comparably on the S\&P 500 options market, both fitting the data very well. In contrast, we find that there are dates when the SVJ model struggles to fit the VIX IVs in addition to the S\&P $500 \mathrm{IVs}$, whereas the SVJ2 model satisfactorily fits both. ${ }^{22}$ Therefore, we conclude from our calibration exercise that we can discard the Heston model from further analysis and that jumps in the volatility are necessary.

Daily calibration is a multiple curve fitting exercise, which matches a model to risk-neutral distributions implied by option prices at different maturities. Some of the parameters we get from daily calibrations are unstable and vary substantially from one day to the next. ${ }^{23}$ To achieve a more robust estimation, consistent with the whole time series of in-sample data, we choose a methodology based on particle filtering.

We find that irrespective of the day, the SVJ specification performs as well as the SVJ2 and SVJ3 specifications on the S\&P 500 options market, all fitting the data very well. In contrast, we find that there are dates when the SVJ model struggles to fit the VIX IVs in addition to the S\&P 500 IVs, whereas the SVJ2 and SVJ3 models satisfactorily fit both. ${ }^{24}$ Therefore, we conclude from our calibration exercise that we can discard the Heston model from further analysis and that jumps in the volatility are necessary.

\footnotetext{
${ }^{22}$ Our findings are consistent with Gatheral (2008), who shows that the Heston model is incapable of reproducing the positive skew in VIX IVs, and with Sepp (2008a,b), who finds that incorporating positive jumps in the volatility dynamics into the Heston model removes this shortcoming.

${ }^{23}$ Parameters obtained when calibrating to daily options prices are not stable over time, as explained in Broadie, Chernov, and Johannes (2007) and Lindström, Ströjby, Brodén, Wiktorsson, and Holst (2008).

${ }^{24}$ Our findings are consistent with Gatheral (2008), who shows that the Heston model is incapable of reproducing the positive skew in VIX IVs, and with Sepp (2008a,b), who finds that incorporating positive jumps in the volatility dynamics into the Heston model removes this shortcoming.
} 


\section{E. Specific data treatment for the particle filter}

As the datasets comprise a large number of options (up to 600 a day), it is unfeasible to calculate the option prices every day for every particle. As a consequence, we follow Pan (2002) and Johannes, Polson, and Stroud (2009), among others, and use weekly (Wednesday) options data. Furthermore, this eliminates beginning-of-week and end-of-week effects. Our particle filter uses daily time steps and incorporates information on the underlying indexes on a daily basis (i.e., only options are considered weekly).

Moreover, the S\&P 500 options dataset contains a large amount of ATM options compared to OTM and deep OTM options. If we use the filter (within the maximum likelihood procedure) on this entire dataset, the fitting of ATM options will be its priority rather than (deep) OTM options. This results in fitting the body of the $\mathrm{S} \& \mathrm{P} 500$ returns distribution rather than the tails, which is not what we want. We need information about the extreme events contained in the data to be incorporated into the models. For this reason, we interpolate the S\&P 500 IV slices and re-sample the option prices from the resulting parametric fit uniformly with respect to moneyness. ${ }^{25}$ Other advantages of our use of interpolation is that the resulting data is arbitrage free and we have fewer points for each slice (but still accurately representing the information of each slice), thus reducing the computational complexity. ${ }^{26}$

For the interpolation, we use the efficient mixture of log-normals approach of Rebonato and Cardoso (2004) to have a parametric fit for each S\&P 500 implied volatility slice. The RMSE of the S\&P 500 implied volatilities parametric fits are on average around $0.25 \%$ and we therefore do not lose information, especially given the market bid-ask spread. Finally, using the parametric fit, we can sample a fixed number (we have chosen 15) of "market option prices" for the desired strikes. We have chosen to resample the option prices from each parametric slice uniformly in the strike (or, equivalently, the moneyness). We however do not resample the options for which the strike is smaller than $40 \%$ or larger than $140 \%$ of the current futures price. The reason is that there are usually only one or two options outside this interval of moneyness and we do not wish to re-sample options where

\footnotetext{
${ }^{25}$ It is common to interpolate data, see, e.g., Broadie, Chernov, and Johannes (2007). This eliminates arbitrage opportunities in the data and removes the accumulation of options around the ATM region.

${ }^{26}$ Since we have considered mid-prices and because of synchronization issues between the underlying and the options, implied volatility slices are not guaranteed to be arbitrage free.
} 
the interpolation results could be driven by an outlier.

We do not perform any interpolation for the VIX options dataset, as most VIX options are OTM and therefore contain information about the tails of the VIX distribution (i.e., variance and central tendency processes). Therefore all available VIX option prices are used.

Finally, we decompose the time series of observations into two periods. The first period is from March 1st, 2006 to Feb 28, 2009 (shortly after the VIX index increased to its highest point). This was a rather calm period that we will use as the in-sample estimation period. ${ }^{27}$ Our out-of-sample period starts on March 1st, 2009 and ends on April 30, 2016. This period includes very high levels of volatility (for implied volatilities from the S\&P 500 and VIX options as well as the VIX index values). The last column of Table 5 presents the number of options within each moneyness and maturity range in both periods. In particular, in the in-sample period our data contains 4'997 closeto-maturity OTM options on the S\&P 500 and 2'283 OTM call options on the VIX. These options have maturities shorter than two months. Analogously, in the out-of-sample period, the dataset contains 27'615 close-to-maturity options on the S\&P 500 and 6'994 on the VIX. As highlighted in Bollerslev and Todorov (2011), these options provide valuable information on jumps as they have little value unless a large movement in the S\&P 500 is possible.

\section{F. Particle filter}

\section{F.1. Measurement equations}

We discretize the continuous-time model on a uniform time grid composed of $M+1$ points $t \in\left\{t_{0}=0, t_{1}=\Delta t, \ldots, t_{k}=k \Delta t, \ldots, t_{M}=M \Delta t\right\}$, for some $M \in \mathbb{N}^{*}$. Since we use daily data, $\Delta t$

\footnotetext{
${ }^{27}$ We have decided to include the beginning of the financial crisis so that the in-sample period actually includes several dates with extreme events.
} 
corresponds to one day. In discrete time, the model evolves under $\mathbb{P}$ as follows:

$$
\begin{aligned}
\Delta Y_{t}= & {\left[-\lambda^{(-)}\left(v_{t}, m_{t}, u_{t}\right)\left(\theta_{Z}^{\mathbb{P}}(0,1,0,0)-1\right)-\lambda^{(+)}\left(v_{t}, m_{t}\right)\left(\theta_{Z}^{\mathbb{P}}(1,0,0,0)-1\right)-\frac{1}{2} v_{t}+\gamma_{t}\right] \Delta t } \\
& +\sqrt{v_{t}} \Delta W_{t}^{Y, \mathbb{P}}+Z_{t}^{Y(+), \mathbb{P}} \Delta N_{t}^{(+)}+Z_{t}^{Y(-), \mathbb{P}} \Delta N_{t}^{(-)} \\
\Delta v_{t}= & \kappa_{v}^{\mathbb{P}}\left(\frac{\kappa_{v}}{\kappa_{v}^{\mathbb{P}}} m_{t}-v_{t}\right) \Delta t+\sigma_{v} \sqrt{v_{t}} \Delta W_{t}^{v, \mathbb{P}}+Z_{t}^{v(-), \mathbb{P}} \Delta N_{t}^{(+)}+Z_{t}^{v(+), \mathbb{P}} \Delta N_{t}^{(-)} \\
\Delta m_{t}= & \kappa_{m}^{\mathbb{P}}\left(\theta_{m}^{\mathbb{P}}-m_{t}\right) \Delta t+\sigma_{m} \sqrt{m_{t}} \Delta W_{t}^{m, \mathbb{P}} \\
\Delta u_{t}= & \kappa_{u}\left(\theta_{u}-u_{t}\right) \Delta t+\sigma_{u} \sqrt{m_{t}} \Delta W_{t}^{u, \mathbb{P}}
\end{aligned}
$$

where the notation $\Delta X_{t}$ for some process $X$ represents the increment $X_{t_{k+1}}-X_{t_{k}}$ with $t=t_{k} \in$ $\left\{t_{0}, \ldots, t_{M-1}\right\}$. In what follows, we will assume that the long-term mean of the process $u_{t}$ is normalized to one, i.e., $\theta_{u}=1$.

As the log-returns are observable, Eq. (F.1) is the first measurement equation. The second measurement equation comes from the observation of the VIX index level with error:

$$
\mathrm{VIX}_{t}^{2}-\left(\alpha_{\mathrm{VIX}^{2}} v_{t}+\beta_{\mathrm{VIX}^{2}} m_{t}+\gamma_{\mathrm{VIX}^{2}} u_{t}+\delta_{\mathrm{VIX}^{2}}\right)=\epsilon_{t}^{\mathrm{VIX}}
$$

Jiang and Tian (2007) point to systematic biases in the calculation of the VIX index, such as model misspecification or data limitations. For example, in practice, the index is calculated using a finite number of options thereby inducing an error in the computation of the integral defining VIX ${ }^{2}$. These biases are captured by the error term $\epsilon_{t}^{\mathrm{VIX}}$, which is assumed to follow a normal distribution with mean zero and variance $s>0, \epsilon_{t}^{\mathrm{VIX}} \sim N(0, s)$.

To better identify the total variance of S\&P 500 returns under the $\mathbb{P}$ measure, we add a measurement equation, which links the logarithm of the daily Realized Variance $\left(\mathrm{RV}_{t}\right)$ of S\&P 500 returns ${ }^{28}$ to the logarithm of the total spot variance under $\mathbb{P}$, as in Filipović, Gourier, and Mancini (2016). The associated measurement error $\epsilon_{t}$ is conditionally normally distributed with mean $\rho_{\epsilon} \epsilon_{t-1}$ and variance $c_{0}+c_{1} \mathrm{RV}_{t-1}$. The rationale behind this component of the measurement equation is the following. Andersen, Bollerslev, Diebold, and Ebens (2001), among others, provide empirical evidence that $\log \left(\mathrm{RV}_{t}\right)$ is approximately normally distributed. The conditional mean specification of

\footnotetext{
${ }^{28}$ The Realized Variance (RV) of the S\&P 500 index is obtained from the website of the Oxford-Man Institute Realized Library.
} 
$\epsilon_{t}$ allows for autocorrelation in the measurement error, which can be induced by clustering of price jumps caused by persistence of the price jump intensity and/or microstructure noise in the estimates of daily realized variance. Autocorrelation in the measurement error is also reported in Wu (2011). The conditional variance specification of $\epsilon_{t}$ captures in a parsimonious way the heteroscedasticity of the measurement error due to the volatility of realized variance.

The last measurements are the prices of S\&P 500 and VIX options. We assume that the option prices are observed with an error. This error represents several sources of noise, such as bid-ask spreads, timing, and processing errors. We define these errors as the relative differences between market $O_{t}^{\mathcal{M}, M k t}$ and model prices $O_{t}^{\mathcal{M}, M o d}, \mathcal{M} \in\{\mathrm{SPX}, \mathrm{VIX}\}$ :

$$
\begin{aligned}
& \frac{O_{t, i}^{\mathrm{SPX}, M o d}\left(Y_{t}, v_{t}, m_{t}, \Theta, \Theta^{\mathbb{P}, \mathbb{Q}}\right)-O_{t, i}^{\mathrm{SPX}, M k t}}{O_{t, i}^{\mathrm{SPX}, M k t}}=\epsilon_{t, i}^{\mathrm{SPX}, \text { options }}, \quad i=1, \ldots, N_{\mathrm{SPX}, t}, \\
& \frac{O_{t, j}^{\mathrm{VIX}, M o d}\left(v_{t}, m_{t}, \Theta, \Theta^{\mathbb{P}, \mathbb{Q}}\right)-O_{t, j}^{\mathrm{VIX}, M k t}}{O_{t, j}^{\mathrm{VIX}, M k t}}=\epsilon_{t, j}^{\mathrm{VIX}, \text { options }}, \quad j=1, \ldots, N_{\mathrm{VIX}, t},
\end{aligned}
$$

where $N_{\mathcal{M}, t}$ is the number of contracts available in the corresponding market and the $\Theta$ 's are the sets of parameters to estimate:

$$
\begin{aligned}
\Theta^{\mathbb{P}} & =\left\{\kappa_{v}^{\mathbb{P}}, \kappa_{m}^{\mathbb{P}}, \theta_{m}^{\mathbb{P}}, \nu_{m}^{\mathbb{P}}, \nu_{v}^{(+) \mathbb{P}}, \nu_{v}^{(-) \mathbb{P}}, \mu_{Y}^{(+) \mathbb{P}}, \mu_{Y}^{(-) \mathbb{P}}, \eta_{Y}\right\}, \\
\Theta & =\left\{\kappa_{v}, \kappa_{m}, \theta_{m}, \nu_{v}^{(+)}, \nu_{v}^{(-)}, \mu_{Y}^{(+)}, \mu_{Y}^{(-)}\right\}, \\
\Theta^{\mathbb{P}, \mathbb{Q}} & =\left\{\lambda_{0}^{(-)}, \lambda_{1}^{(-)}, \lambda_{2}^{(-)}, \lambda_{3}^{(-)}, \lambda_{0}^{(+)}, \lambda_{1}^{(+)}, \lambda_{2}^{(+)}, \sigma_{m}, \sigma_{v}, \sigma_{u}, \kappa_{u}, \rho_{Y v}\right\} .
\end{aligned}
$$

We assume the error terms to be normally distributed and heteroscedastic:

$$
\epsilon_{t, i}^{\mathrm{SPX}, \text { options }} \sim \mathcal{N}\left(0, \sigma_{\epsilon_{t, i}^{\mathrm{SPX}}}^{2}\right), \quad \epsilon_{t, j}^{\mathrm{VIX}, \text { options }} \sim \mathcal{N}\left(0, \sigma_{\epsilon_{t, j}^{\mathrm{VIX}}}^{2}\right)
$$

The variance of the errors is

$$
\begin{aligned}
\sigma_{\epsilon_{t, i}^{\mathrm{SPX}}}^{2} & =\exp \left(\phi_{0} \cdot \text { bid-ask } \operatorname{spread}_{i}+\phi_{1}\left|\log \left(\frac{K_{\mathrm{VIX}, i}}{F_{t}^{\mathrm{SPX}}\left(T_{i}\right)}\right)\right|+\phi_{2}\left(T_{i}-t\right)+\phi_{3}\right), \\
\sigma_{\epsilon_{t, j}^{\mathrm{VIX}}}^{2} & =\exp \left(\psi_{0} \cdot \text { bid-ask spread }_{j}+\psi_{1}\left|\log \left(\frac{K_{\mathrm{SPX}, j}}{F_{t}^{\mathrm{VIX}}\left(T_{j}\right)}\right)\right|+\psi_{2}\left(T_{j}-t\right)+\psi_{3}\right),
\end{aligned}
$$


with $\phi_{i}$ and $\psi_{i}$ in $\mathbb{R}, i \in\{0,1,2,3\} .^{29}$

\section{F.2. Filtering methodology}

We follow the notation used in Section 4. The log-likelihood of a time series of $n+1$ observations with joint density $p$, conditional on a set of parameters $\Theta$, is equal to

$$
\log p\left(y^{t_{n}} \mid \Theta\right)=\log p\left(y_{t_{0}}, \ldots, y_{t_{n}} \mid \Theta\right)=\sum_{k=1}^{n} \log p\left(y_{t_{k}} \mid y^{t_{k-1}}, \Theta\right)+\log p\left(y_{t_{0}} \mid \Theta\right)
$$

where, by the Law of Total Probability,

$$
p\left(y_{t_{k}} \mid y^{t_{k-1}}, \Theta\right)=\int p\left(y_{t_{k}} \mid L_{t_{k}}, \Theta\right) p\left(L_{t_{k}} \mid y^{t_{k-1}}, \Theta\right) d L_{t_{k}} .
$$

Given an initial density $p\left(L_{t_{0}} \mid \Theta\right)$, the transition density of the state variables $p\left(L_{t_{k}} \mid L_{t_{k-1}}, \Theta\right)$ and the likelihood function $p\left(y_{t_{k}} \mid L_{t_{k}}, \Theta\right)$, filtering methods allow us to estimate the distribution $p\left(L_{t_{k}} \mid y^{t_{k}}, \Theta\right)$ of the current state at time $t_{k}=k \Delta t$, given all observations up to that time. In the following, we simplify notation and drop the subscript for the conditioning on the parameters $\Theta$. The filtering density is given by Bayes's formula,

$$
p\left(L_{t_{k}} \mid y^{t_{k}}\right) \propto p\left(y_{t_{k}} \mid L_{t_{k}}\right) p\left(L_{t_{k}} \mid y^{t_{k-1}}\right)
$$

where $\propto$ means proportional to.

The likelihood function is known, but the predictive distribution of the state is not. It is given by the following integral, which involves the previous filtering density:

$$
p\left(L_{t_{k}} \mid y^{t_{k-1}}\right)=\int p\left(L_{t_{k}} \mid L_{t_{k-1}}\right) p\left(L_{t_{k-1}} \mid y^{t_{k-1}}\right) d L_{t_{k-1}}
$$

The key idea is to approximate the posterior density function of the latent variables $p\left(L_{t_{k}} \mid y^{t_{k}}\right)$ by a

\footnotetext{
${ }^{29}$ The fact that the option pricing errors are normally distributed does not constitute a restriction. The reason is that the errors are heteroscedastic and the coefficients generating the heteroscedasticity are driven by the data, i.e., we optimize over the parameters $\left\{\phi_{i}, \psi_{i}\right\}_{0 \leq i \leq 3}$.
} 
sum of point masses positioned at strategic points, called particles, $\left\{L_{t_{k}}^{(i)}\right\}_{1 \leq i \leq n_{p}}$ :

$$
\hat{p}\left(L_{t_{k}} \mid y^{t_{k}}\right)=\sum_{i=1}^{n_{p}} \pi_{t_{k}}^{(i)} \delta\left(L_{t_{k}}-L_{t_{k}}^{(i)}\right),
$$

where $\pi_{t_{k}}^{(i)}$ denotes the normalized importance weight for particle $i, \delta($.$) is the Dirac delta function,$ and $n_{p}$ is the number of support points (particles) for $\hat{p}\left(L_{t_{k}} \mid y^{t_{k}}\right)$. Then, we can recursively calculate the filtering density by

$$
\begin{aligned}
\hat{p}\left(L_{t_{k}} \mid y^{t_{k}}\right) & \propto \int p\left(y_{t_{k}} \mid L_{t_{k}}\right) p\left(L_{t_{k}} \mid L_{t_{k-1}}\right) \hat{p}\left(L_{t_{k-1}} \mid y^{t_{k-1}}\right) d L_{t_{k-1}} \\
& =\sum_{i=1}^{n_{p}} p\left(y_{t_{k}} \mid L_{t_{k}}\right) p\left(L_{t_{k}} \mid L_{t_{k-1}}^{(i)}\right) \pi_{t_{k-1}}^{(i)} .
\end{aligned}
$$

To implement the particle filter, we need to simulate at every time $t_{k}$ a number $n_{p}$ of particles $L_{t_{k}}^{(i)}, i=1, \ldots, n_{p}$ from $p\left(L_{t_{k}} \mid y^{t_{k-1}}\right)$ and to evaluate $p\left(y_{t_{k}} \mid L_{t_{k}}^{(i)}\right)$. Based on these simulated particles, we can approximate $p\left(y_{t_{k}} \mid y^{t_{k-1}}\right)$ by

$$
p\left(y_{t_{k}} \mid y^{t_{k-1}}\right) \approx \frac{1}{n_{p}} \sum_{i=1}^{n_{p}} p\left(y_{t_{k}} \mid L_{t_{k}}^{(i)}\right) .
$$

We used $n_{p}=30,000$ particles on days when the observations contain option prices and $n_{p}=$ 10, 000 when the observations are only composed of the S\&P500 returns and VIX index levels. Larger numbers of particles did not change our estimates, but increased the computational burden.

The filtering algorithm can be decomposed into the following steps.

Step 1: Initialization. We simulate $n_{p}$ initial particles for the latent variables $\left\{v_{t_{0}}^{(i)}, m_{t_{0}}^{(i)}, u_{t_{0}}^{(i)}\right\}_{i=1, \ldots, n_{p}}$, which are compatible with the initial value of the VIX squared, i.e., given the specification in Eq. (F.5). The following steps are repeated for each time step $t_{k}$ in the grid from $k=0$ to $k=M-1$.

Step 2: First-stage resampling. At this point, we assume that we have $n_{p}$ particles (i.e., possible values of $m_{t}, v_{t}$, and $u_{t}$ ) at time $t_{k}$ given all observations $y^{t_{k}}$ up to $t_{k}$. At time $t_{k+1}$, there are new observations $y_{t_{k+1}}$. The goal of this step is to retain, from the previous sample of particles $\left\{v_{t_{k}}^{(i)}, m_{t_{k}}^{(i)}, u_{t_{k}}^{(i)}\right\}_{1 \leq i \leq n_{p}}$, only those which are likely to generate the new observations $y_{t_{k+1}}$. For this 
purpose, we assign a weight (the so called "first-stage weights") to each particle, which is proportional to the likelihood of new market observations $y_{t_{k+1}}$ given the value of the particle $L_{t_{k}}$ at time $t_{k}$. Intuitively, particles that are compatible with the new observations will be assigned larger weights than other particles. To increase the speed of the first-stage resampling, we do not consider options as part of the observations $y_{t_{k+1}}$ (only in this step) and limit $y_{t_{k+1}}$ to the values of the indexes.

The first stage weight $\omega_{t_{k+1}}^{(i)}$ assigned to the $i^{\text {th }}$ particle $L_{t_{k}}^{(i)}$ at time $t_{k+1}$ is given by

$$
\omega_{t_{k+1}}^{(i)}=p\left(L_{t_{k}}^{(i)} \mid y_{t_{k+1}}\right) \propto p\left(y_{t_{k+1}} \mid L_{t_{k}}^{(i)}\right)
$$

where $p\left(y_{t_{k+1}} \mid L_{t_{k}}^{(i)}\right)$ is the density of the observation vector $y_{t_{k+1}}$ given the values of the particle vector $L_{t_{k}}^{(i)}$. The importance weights $\left\{\omega_{t_{k+1}}^{(i)}\right\}_{1 \leq i \leq n_{p}}$ add up to 1 , so that they define a proper probability mass function. Conditioning on the number of jumps in $\Delta Y_{t}$ (or equivalently in $\Delta v_{t}$ ) and $\Delta m_{t}$ gives

$$
\omega_{t_{k+1}}^{(i)} \propto \sum_{j, l \in \mathbb{N}} p\left(y_{t_{k+1}} \mid L_{t_{k}}^{(i)}, \Delta N_{t_{k}}^{(+)}, \Delta N_{t_{k}}^{(-)}\right) \mathbb{P}\left(\Delta N_{t_{k}}^{(+)}=j, \Delta N_{t_{k}}^{(-)}=l\right) .
$$

Given that we use daily observations, we limit the possible number of jumps of the Poisson random variables $\Delta N_{t_{k}}^{(+)}$and $\Delta N_{t_{k}}^{(-)}$to zero or one. ${ }^{30}$ We recall that the new observation is composed of the $\mathrm{S} \& \mathrm{P} 500$ returns and the VIX level $y_{t_{k+1}}=\left(\Delta Y_{t_{k}}=Y_{t_{k+1}}-Y_{t_{k}}, \mathrm{VIX}_{t_{k+1}}^{2}\right)$. Since the $\mathrm{VIX}_{t_{k+1}}^{2}$ is a sum of normal distributions and no more than two exponential distributions, there is no closed form for this bivariate density in the general case. To preserve tractability, we approximate the exponentially distributed jump sizes by a categorical distribution (a generalization of a Bernoulli distribution), which is supported in a certain number of (the corresponding exponential distribution's) quantiles. ${ }^{31}$ As a consequence, the weight $\omega_{t_{k+1}}^{(i)}$ is a sum of weighted bivariate normal densities.

To eliminate the particles $\left\{L_{t_{k}}^{(i)}\right\}_{1 \leq i \leq n_{p}}$ that are not likely to generate the new observations $y_{t_{k+1}}$, we resample (with replacement) the particles according to a stratified resampling scheme: ${ }^{32}$

$$
z(i) \sim \operatorname{StratRes}\left(n_{p}, \omega_{t_{k+1}}^{(1)}, \ldots, \omega_{t_{k+1}}^{\left(n_{p}\right)}\right)
$$

\footnotetext{
${ }^{30}$ This Bernoulli approximation is found to be very accurate in Johannes, Polson, and Stroud (2009).

${ }^{31}$ Robustness tests were performed on simulated data to check that the choice of quantiles was appropriate.

${ }^{32}$ We checked that using a multinomial or stratified resampling scheme gives similar results.
} 
We have now new sample of $n_{p}$ latent factors $\left\{L_{t_{k}}^{(j)}\right\}_{1 \leq j \leq n_{p}}$, which are now equally likely. Indeed, particles representing $m_{t_{k}}, v_{t_{k}}$, and $u_{t_{k}}$ are shuffled into a new set of particles: $\left\{m_{t_{k}}^{(j)}, v_{t_{k}}^{(j)}, u_{t_{k}}^{(j)}\right\}_{j=1 . . n_{p}}=$ $\left\{m_{t_{k}}^{z(i)}, v_{t_{k}}^{z(i)}, u_{t_{k}}^{z(i)}\right\}_{i=1 . . n_{p}}$. We resample the same number of particles, although this is in principle not necessary.

The next step of the particle filter consists in propagating the latent factors according to their conditional density given the previous values $L_{t_{k}}^{(i)}$ and the new observations $y_{t_{k+1}}$ :

$$
L_{t_{k+1}}^{(i)} \sim p\left(L_{t_{k+1}} \mid L_{t_{k}}^{(i)}, y_{t_{k+1}}\right)
$$

Because the distribution $p\left(L_{t_{k+1}} \mid L_{t_{k}}^{(i)}, y_{t_{k+1}}\right)$ is not known in closed form, we use a proposal density $q\left(L_{t_{k+1}} \mid L_{t_{k}}^{(i)}, y_{t_{k+1}}\right)$. Propagating $v_{t}, m_{t}$, and $u_{t}$ requires preliminary knowledge on the jump components, so we first focus on the jumps.

Step 3: Generating the jumps. We calculate the joint probability of jumps in $\Delta Y_{t}$ (or equivalently in $\Delta v_{t}$ ) between $t_{k}$ and $t_{k+1}$ using

$$
\mathbb{P}\left(\Delta N_{t_{k}}^{(+)}, \Delta N_{t_{k}}^{(-)} \mid y_{t_{k+1}}\right) \propto p\left(y_{t_{k+1}} \mid \Delta N_{t_{k}}^{(+)}, \Delta N_{t_{k}}^{(-)}\right) \mathbb{P}\left(\Delta N_{t_{k}}^{(+)}, \Delta N_{t_{k}}^{(-)}\right)
$$

Conditionally on the jump sizes, the first part of the right-hand side has already been calculated in the first-stage weights. Using Bayes's rule, we get an approximation for $\mathbb{P}\left(\Delta N_{t_{k}}^{(+)}, \Delta N_{t_{k}}^{(-)} \mid y_{t_{k+1}}\right)$.

We simulate the jump sizes for $\Delta v_{t}$ and $\Delta m_{t}$ according to their exponential law.

Step 4: Propagating the latent factors. The latent factors $v, m$, and $u$ are propagated following a Milstein discretization scheme of the SDE. See Kloeden and Platen (1992) for details. We use the full truncation method to prevent them from taking negative values.

Step 5: Computing the filtering density. At this point, the newly generated particles $\left\{L_{t_{k+1}}^{(i)}\right\}_{1 \leq i \leq n_{p}}$ are a sample of $p\left(L_{t_{k+1}} \mid y^{t_{k+1}}\right)$. We now calculate the second-stage weights $\left\{\pi_{t_{k+1}}^{(i)}\right\}_{1 \leq i \leq n_{p}}$ which approximate the probabilities $p\left(L_{t_{k+1}}^{(i)} \mid y^{t_{k+1}}\right)$, and give an approximation for the filtering density at time $t_{k+1}$. These weights are proportional to the likelihood of observations at time $t_{k+1}$ given the 
propagated particles $L_{t_{k+1}}^{(i)}$, with a correction related to the proposal density

$$
\pi_{t_{k+1}}^{(i)} \propto \frac{p\left(L_{t_{k+1}}^{(i)} \mid L_{t_{k}}^{(i)}\right) p\left(y_{t_{k+1}} \mid L_{t_{k+1}}^{(i)}\right)}{\omega_{t_{k+1}}^{z(i)} q\left(L_{t_{k+1}}^{(i)} \mid L_{t_{k}}^{(i)}, y_{t_{k+1}}\right)}
$$

The posterior distribution of the state variables is approximated by

$$
\hat{p}\left(L_{t_{k+1}} \mid y^{t_{k+1}}\right)=\sum_{i=1}^{n_{p}} \pi_{t_{k+1}}^{(i)} \delta\left(L_{t_{k+1}}-L_{t_{k+1}}^{(i)}\right) .
$$

We choose the most likely value of a given factor by taking the expectation of the estimated filtering density, e.g., $\hat{v}_{t_{k+1}}=\mathbb{E}_{\hat{p}}\left[v_{t_{k+1}}^{(i)}\right]$.

The algorithm described above extracts latent factors, if one assumes that the model parameters are known. Pitt (2002) builds on Gordon, Salmond, and Smith (1993) to show that the parameters can be estimated using the Maximum Likelihood Importance Sampling Criterion, defined as the product over time of the averages of the second-stage weights. The likelihood of observations given the values of the particles is then estimated by the average of the second-stage weights over the particles

$$
\hat{p}\left(y^{t_{n}} \mid \Theta, \mathcal{M}\right)=\prod_{k=1}^{M} \hat{p}\left(y_{t_{k}} \mid y^{t_{k-1}}, \Theta, \mathcal{M}\right) \hat{p}\left(y_{t_{0}} \mid \Theta, \mathcal{M}\right)
$$

where $\hat{p}\left(y_{t_{k}} \mid y^{t_{k-1}}, \Theta, \mathcal{M}\right)=\frac{1}{n_{p}} \sum_{i=1}^{n_{p}} \pi_{t_{k}}^{(i)}$.

Finally, we use the Weighted Likelihood function analyzed in $\mathrm{Hu}$ and Zidek (2002) to assign comparable weights to the different datasets and to ensure that the estimation is not dominated by S\&P 500 options. Such procedure has been used by Ornthanalai (2014) to estimate a model with Lévy jumps to S\&P 500 options and returns.

\section{G. Additional model diagnostics}

This appendix provides additional diagnostics of model specification for the SVJ3 model, including VIX options in the estimation dataset. In Figure G.1, Panels A and B display the mean of the estimated posterior distribution of the Brownian motions driving the S\&P 500 returns and the short-term variance factor $v$. The filtered Brownian motion driving the returns exhibits excess 
negative skewness, which is probably due to the fact that the jumps captured by the model are large jumps (around -10\%). Due to the financial crisis being in our in-sample period, the model puts emphasis on these catastrophic jumps, which causes it to neglect small negative jumps. These jumps are captured by the Brownian motion instead.

[Fig. G.1 about here.]

We find a correlation of -0.75 between the two filtered Brownian motions, consistently with the estimated value of the leverage coefficient $(-0.74)$. The correlation between the filtered Brownian motions driving $v$ and $m$ is -0.09 . Panel $\mathrm{C}$ of Figure G.1 compares the model-implied VIX values to the data, and shows that the model provides an excellent fit to the VIX data. This is confirmed by small RMSEs. The in-sample RMSE is 0.026 (0.016 if excluding the period starting in September 2008). The out-of-sample RMSE is 0.020. Panel D represents the error between the true VIX squared and the model-implied value across time. The assumption of independent and identically distributed errors is clearly violated. In particular, errors are much larger, consistently with intuition, during high volatility periods. However, even during these periods, the errors remain very small. Panel E of Figure G.1 compares the trajectory of the realized variance of S\&P 500 returns to the estimated trajectory of the model-implied quadratic variation, i.e., total spot variance of returns. There is a satisfactory overlap of the two curves.

Figure G.2 represents, for every month, two measures of realized variation (annualized) computed from high-frequency data, versus the model $\mathbb{P}$-expectation of their limiting quantity at the beginning of the month. Panel A plots the ex-post realized variance and compares it to the expected total variance. Due to averaging over a month, the ex-post variance is smoother than its expectation, which adjusts every day to new information. The two curves are reasonably close. Similarly, Panel B displays the tri-power quarticity versus the expected integrated quarticity. ${ }^{33}$ The tri-power quarticity is an estimate of the integrated quarticity which is robust to jumps, see Barndorff-Nielsen and Shephard (2004). The ex-post realized quarticity tends to be higher than its first conditional moment during the crisis, but smaller otherwise. Overall, these plots do not underline obvious shortcomings of the model, considering the measurement errors made when calculating measures of variation from

\footnotetext{
${ }^{33}$ Our dataset for the tri-power quarticity ends in 2010. Therefore, we only display the comparison between the dataand model implied quarticity until this date.
} 
high-frequency returns.

[Fig. G.2 about here.]

\section{H. Variance risk premium}

The expression of the VRP involves the following terms:

$$
\begin{aligned}
& \mathbb{E}^{\mathbb{Q}}\left(\int_{t}^{T} v_{s} d s\right)=A(T-t) v_{t}+B(T-t) m_{t}+D(T-t) u_{t}+G(T-t), \\
& \mathbb{E}^{\mathbb{Q}}\left(\int_{t}^{T} m_{s} d s\right)=\hat{A}(T-t) m_{t}+\hat{B}(T-t), \\
& \mathbb{E}^{\mathbb{Q}}\left(\int_{t}^{T} u_{s} d s\right)=\bar{A}(T-t) u_{t}+\bar{B}(T-t),
\end{aligned}
$$

where are $A, B, D, G, \hat{A}, \hat{B}, \bar{A}$, and $\bar{B}$ are given by Eqs. (A.6)-(A.9). Expectations under $\mathbb{P}$ are calculated following the same procedure.

To calculate the discontinuous component of the VRP, we use that:

$$
\begin{aligned}
& \mathbb{E}_{t}^{\mathbb{Q}}\left(\sum_{i=N_{t}^{(+/-)}}^{N_{T}^{(+/-)}}\left(Z_{i}^{(+/-)}\right)^{2}\right)=\mathbb{E}_{t}^{\mathbb{Q}}\left(\sum_{i=N_{t}^{(+/-)}}^{N_{T}^{(+/-)}} 2\left(\mu_{Y}^{(+/-)}\right)^{2}\right) \\
& \quad=2\left(\mu_{Y}^{(+/-)}\right)^{2}\left(\lambda_{0}^{(+/-)}(T-t)+\lambda_{1}^{(+/-)} \mathbb{E}^{\mathbb{Q}}\left[\int_{t}^{T} v_{s} d s\right]+\lambda_{2}^{(+/-)} \mathbb{E}^{\mathbb{Q}}\left[\int_{t}^{T} m_{s} d s\right]+\lambda_{3}^{(+/-)} \mathbb{E}^{\mathbb{Q}}\left[\int_{t}^{T} u_{s} d s\right]\right) .
\end{aligned}
$$


Table 1. Descriptive statistics for daily S\&P 500 futures returns and daily VIX returns and square levels for the periods from March 2006 to February 2009, and March 2009 to April 2016. We report the mean (Mean), standard deviation (Std), skewness (Skew), and kurtosis (Kurt).

\begin{tabular}{|c|c|c|c|c|c|c|c|c|}
\hline & \multicolumn{4}{|c|}{ March 2006-February 2009} & \multicolumn{4}{|c|}{ March 2009-April 2016} \\
\hline & Mean & Std & Skew & Kurt & Mean & Std & Skew & Kurt \\
\hline S\&P 500 ret. & -0.0009 & 0.0170 & -0.1487 & 11.5442 & 0.0006 & 0.0107 & -0.0879 & 7.4032 \\
\hline VIX ret. & 0.0048 & 0.0774 & 1.3553 & 10.7419 & 0.0020 & 0.0750 & 1.2731 & 8.1333 \\
\hline $\mathrm{VIX}^{2}$ & 0.0730 & 0.1038 & 2.7325 & 10.7644 & 0.0427 & 0.0341 & 2.2688 & 8.8821 \\
\hline
\end{tabular}


Table 2. Log-likelihood (LL) and values of the AIC and BIC tests for sub-models of the SVJ3 model (dynamics given in (1)-(3)) and for the benchmark model (dynamics given in (11)). The specifications considered are: no jumps (SV2), $m$ and $u$ are constant (SVJ), $u$ is constant (SVJ2), no negative jumps in the variance $\left(\mathrm{SVJ}^{\Delta v>0}\right)$. The benchmark model has two factors and normally distributed jumps in the returns and their variance. The estimation period is from March 2006 to February 2009.

\begin{tabular}{|c|c|c|c|c|c|c|}
\hline & \multicolumn{6}{|c|}{ w/o VIX options } \\
\hline & SV2 & SVJ & SVJ2 & $\mathrm{SVJ}^{\Delta v>0}$ & Benchmark & SVJ3 \\
\hline $\mathrm{LL}$ & 9778 & 10224 & 10424 & 10415 & 10370 & 10473 \\
\hline AIC & -19538 & -20414 & -20802 & -20784 & -20704 & -20898 \\
\hline \multirow[t]{3}{*}{$\mathrm{BIC}$} & -19496 & -20335 & -20695 & -20677 & -20621 & -20787 \\
\hline & & & \multicolumn{2}{|c|}{$\mathrm{w} /$ VIX options } & & \\
\hline & & & SVJ2 & $\mathrm{SVJ}^{\Delta v>0}$ & Benchmark & SVJ3 \\
\hline $\mathrm{LL}$ & & & 9717 & 9729 & 9538 & 9949 \\
\hline AIC & & & -19388 & -19412 & -19040 & -19850 \\
\hline BIC & & & -19281 & -19305 & -18957 & -19739 \\
\hline
\end{tabular}




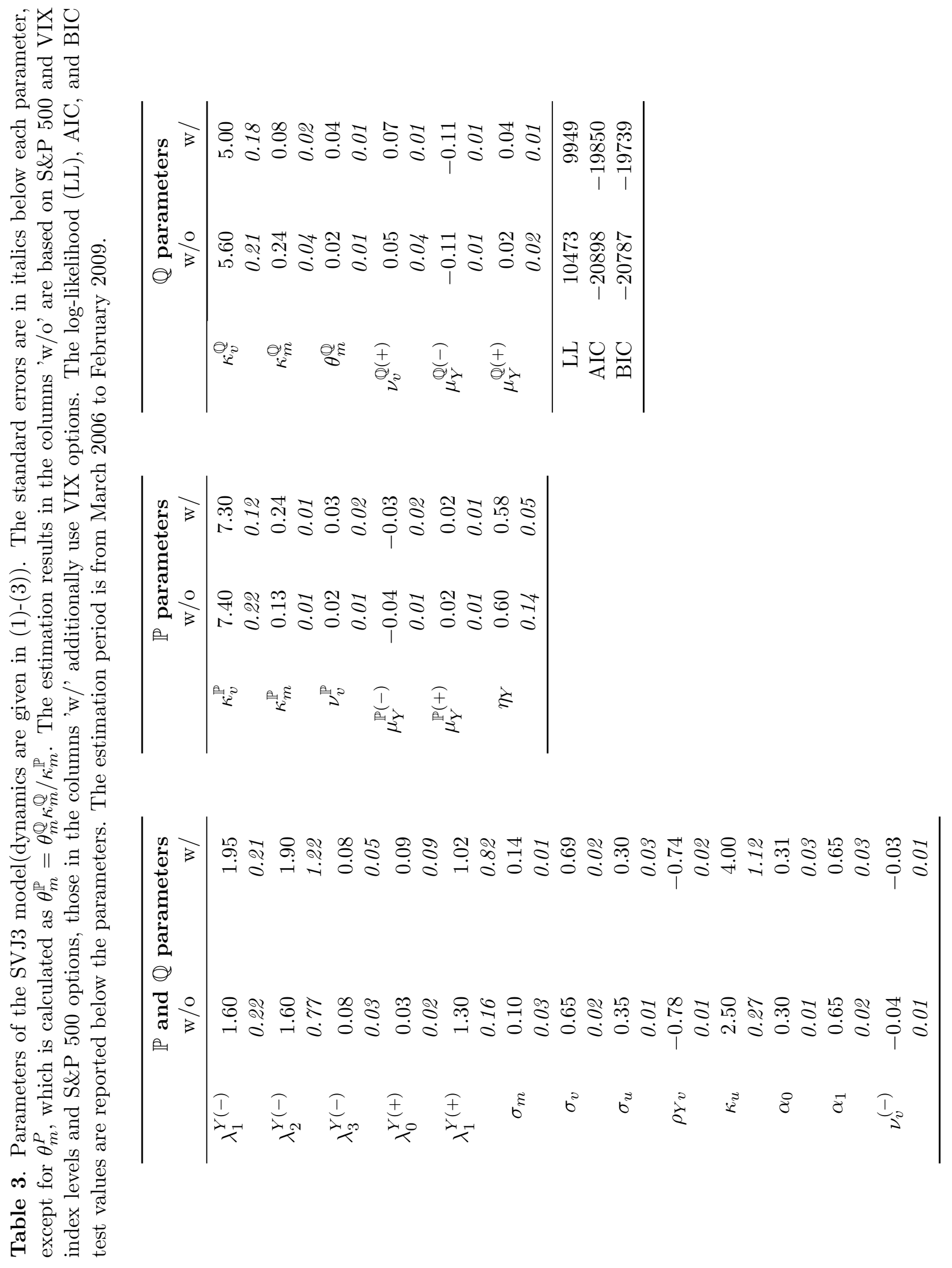




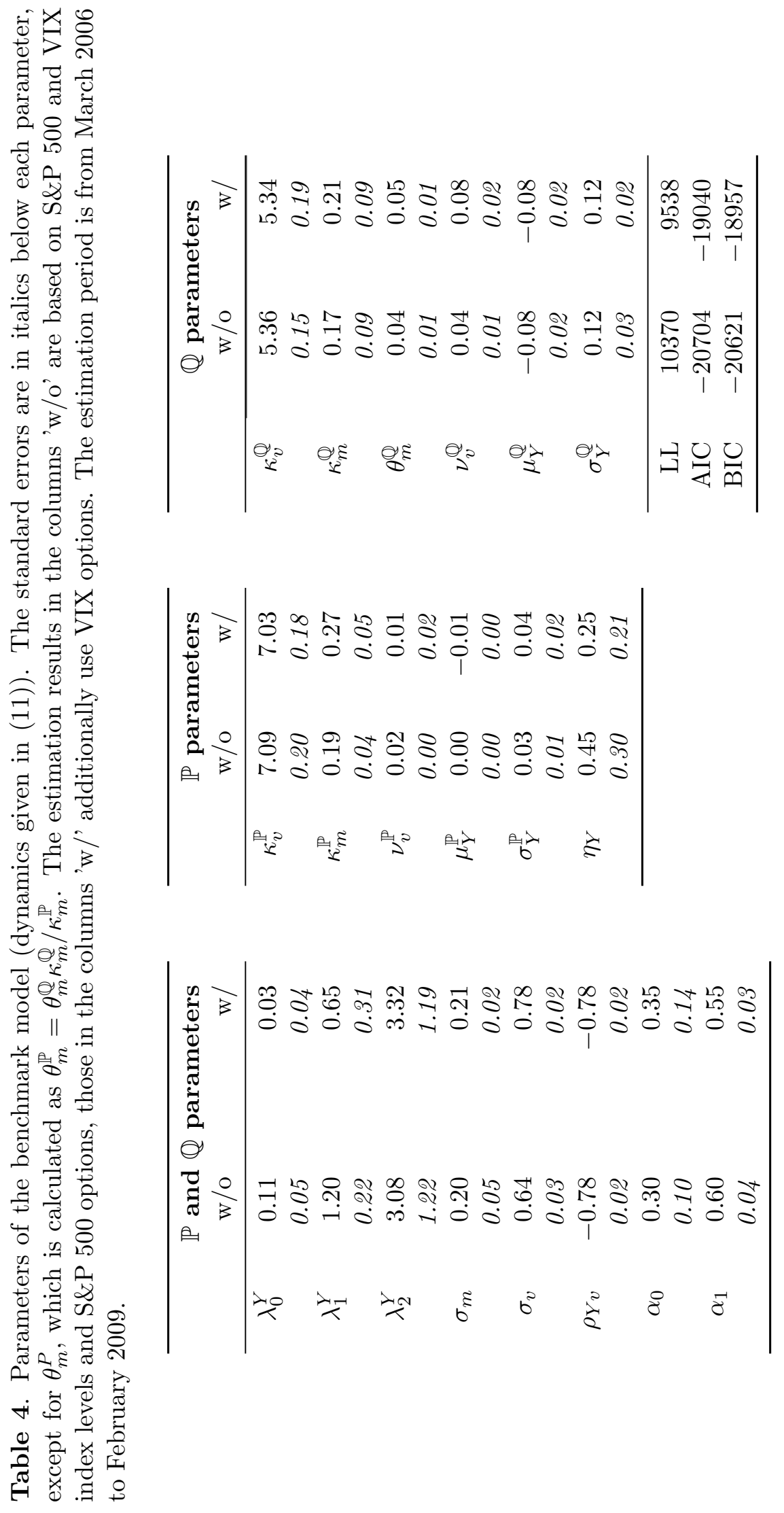




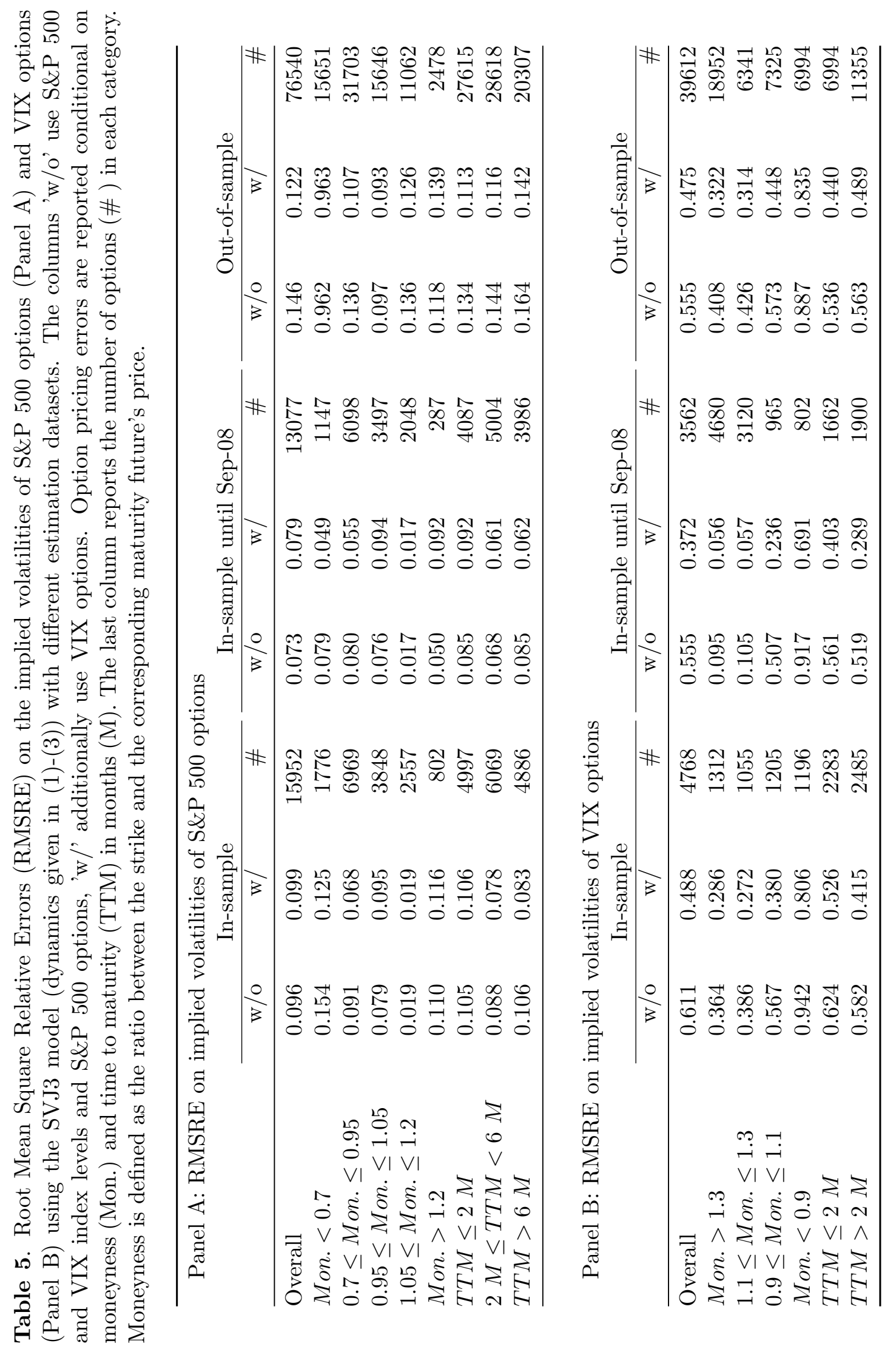




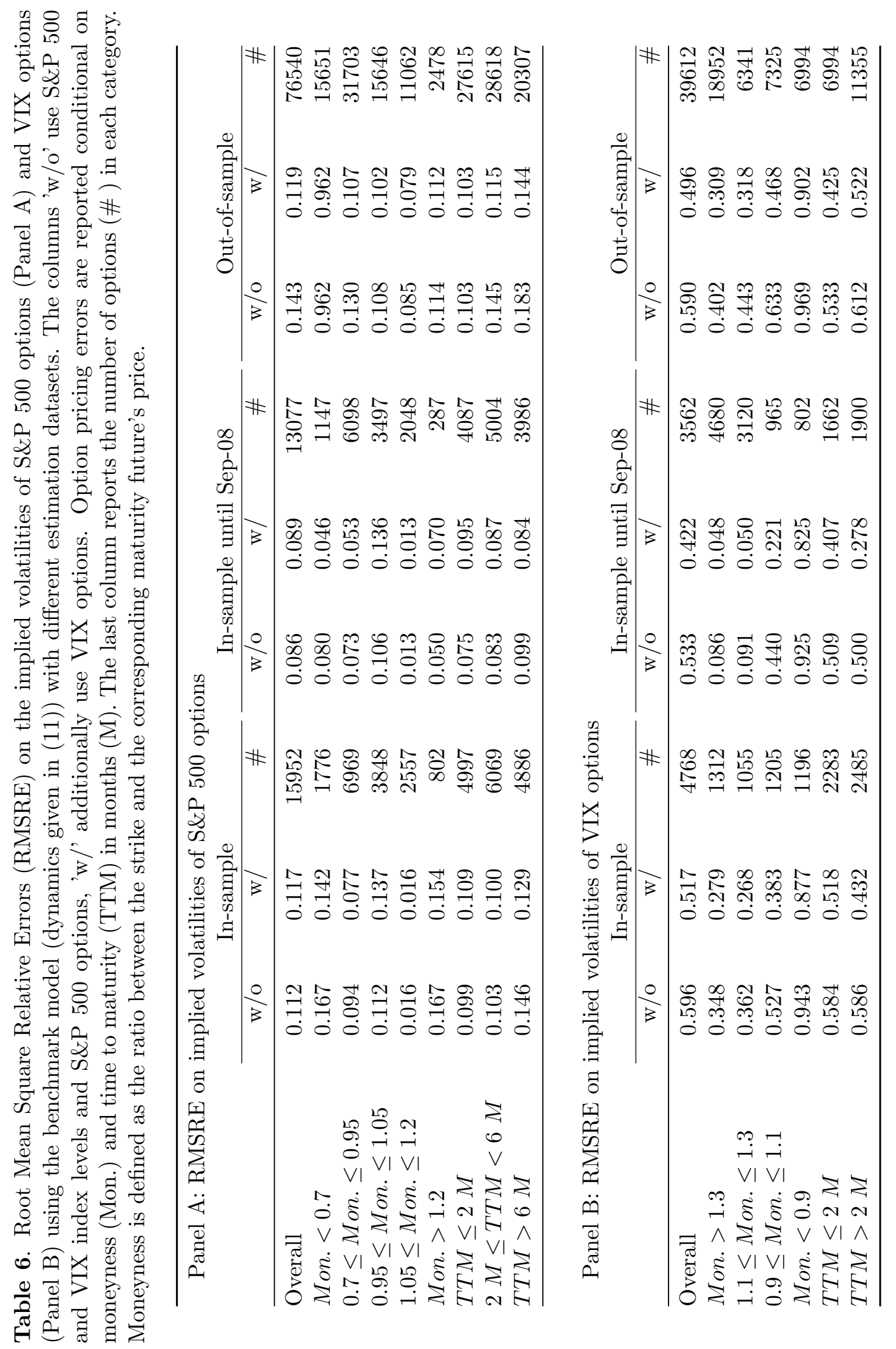


Table 7. Statistics of Diebold-Mariano tests applied to option pricing errors. The pricing error at time $t$ is defined as the mean-square relative error between the model-implied option prices and observed prices. The test statistics compare the errors when excluding VIX options to the case when including VIX options. Under the null hypothesis that the two estimation datasets produce pricing errors of the same magnitude, the Diebold-Mariano test statistic is standard normal. A positive value means that the pricing errors become smaller when including VIX options.

\begin{tabular}{lrr}
\hline & In-sample & Out-of-sample \\
\hline S\&P 500 options & & \\
\hline Overall & 0.908 & 9.488 \\
Mon. $<0.7$ & 0.273 & -6.252 \\
$0.7 \leq$ Mon.$\leq 0.95$ & 0.719 & 3.327 \\
$0.95 \leq$ Mon.$\leq 1.05$ & -0.158 & 0.400 \\
$1.05 \leq$ Mon. $\leq 1.2$ & 0.000 & 0.693 \\
Mon. $>1.2$ & -0.181 & -0.167 \\
TTM $\leq 2 M$ & -0.063 & 3.535 \\
$2 M \leq T T M<6 M$ & 0.338 & 2.712 \\
$T T M>6 M$ & 0.422 & 5.721 \\
\hline
\end{tabular}

\begin{tabular}{lrr}
\hline VIX options & & \\
\hline Overall & 111.753 & 133.872 \\
Mon. $>1.3$ & 7.432 & 26.020 \\
$1.1 \leq$ Mon.$\leq 1.3$ & 3.047 & 6.976 \\
$0.9 \leq$ Mon. $\leq 1.1$ & 3.130 & 15.870 \\
Mon. $<0.9$ & 17.423 & 132.233 \\
$T T M \leq 2 M$ & 13.062 & 23.411 \\
TTM $>2 M$ & 9.928 & 120.845 \\
\hline
\end{tabular}




\begin{tabular}{|c|c|c|c|c|c|c|c|c|}
\hline & \multicolumn{4}{|c|}{ w/o VIX options } & \multicolumn{4}{|c|}{ w/ VIX options } \\
\hline & Overall & IS & IS S08 & OOS & Overall & IS & IS S08 & OOS \\
\hline \multicolumn{9}{|c|}{ SVJ3 model } \\
\hline $2 \mathrm{~m}$ & 0.018 & 0.017 & 0.008 & 0.018 & 0.015 & 0.013 & 0.007 & 0.015 \\
\hline $3 \mathrm{~m}$ & 0.023 & 0.022 & 0.011 & 0.023 & 0.019 & 0.017 & 0.006 & 0.019 \\
\hline $5 \mathrm{~m}$ & 0.029 & 0.028 & 0.015 & 0.030 & 0.024 & 0.021 & 0.007 & 0.024 \\
\hline $6 \mathrm{~m}$ & 0.031 & 0.030 & 0.016 & 0.031 & 0.025 & 0.022 & 0.008 & 0.026 \\
\hline \multicolumn{9}{|c|}{ Benchmark model } \\
\hline $2 \mathrm{~m}$ & 0.017 & 0.018 & 0.016 & 0.015 & 0.014 & 0.014 & 0.007 & 0.014 \\
\hline $3 \mathrm{~m}$ & 0.020 & 0.023 & 0.018 & 0.018 & 0.018 & 0.018 & 0.007 & 0.017 \\
\hline $5 \mathrm{~m}$ & 0.025 & 0.028 & 0.022 & 0.021 & 0.022 & 0.022 & 0.010 & 0.022 \\
\hline $6 m$ & 0.026 & 0.031 & 0.024 & 0.022 & 0.024 & 0.024 & 0.012 & 0.023 \\
\hline
\end{tabular}

Table 8. Root Mean Square Errors on the synthesized squared VIX indices for maturities of two, three, five, and six months, excluding or including VIX options in the estimation dataset. Results are reported over the entire time period (Overall), the in-sample period (IS), the in-sample period until September 2008 (IS S08), the out-of-sample period (OOS). The indices are synthesized from S\&P 500 options. 


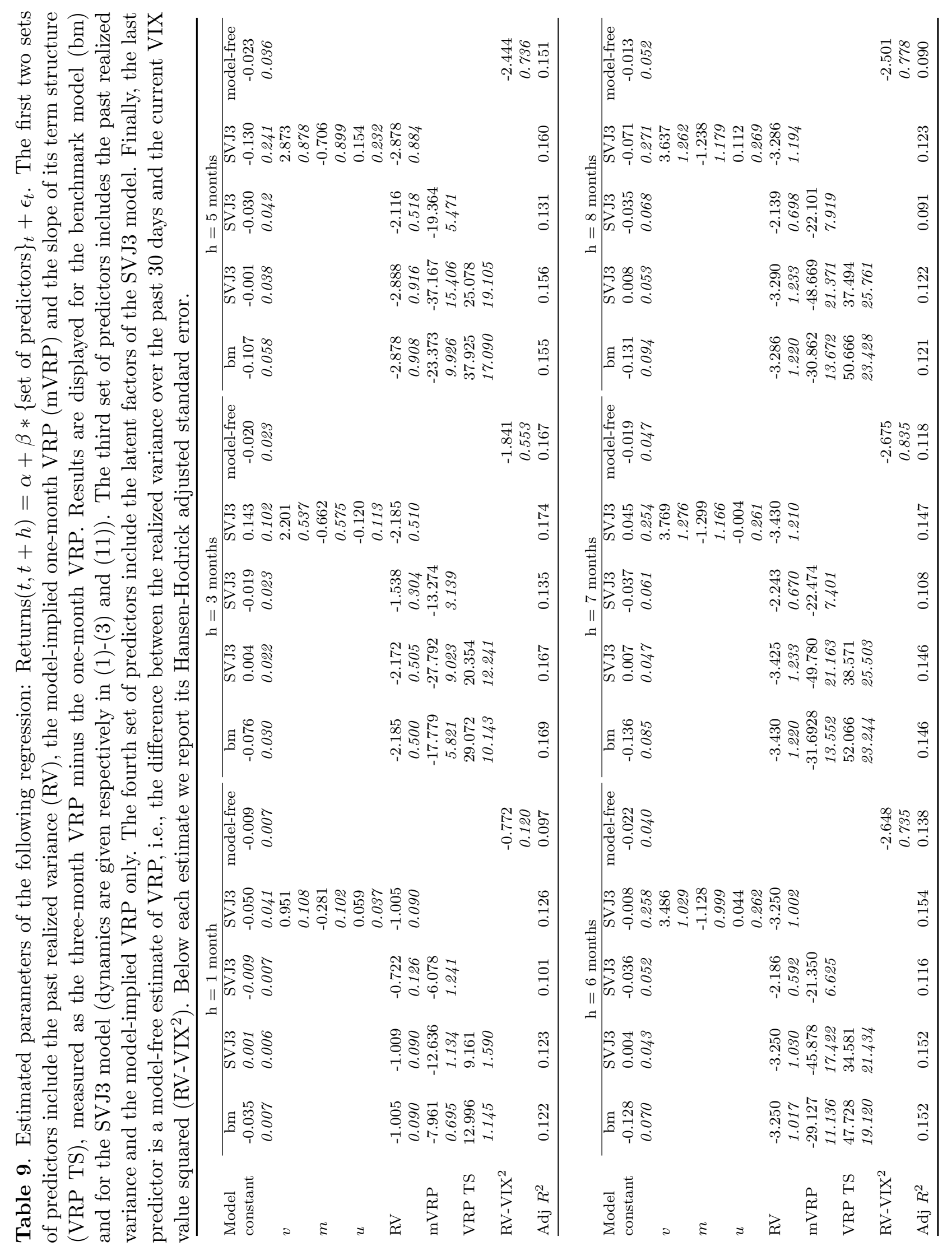




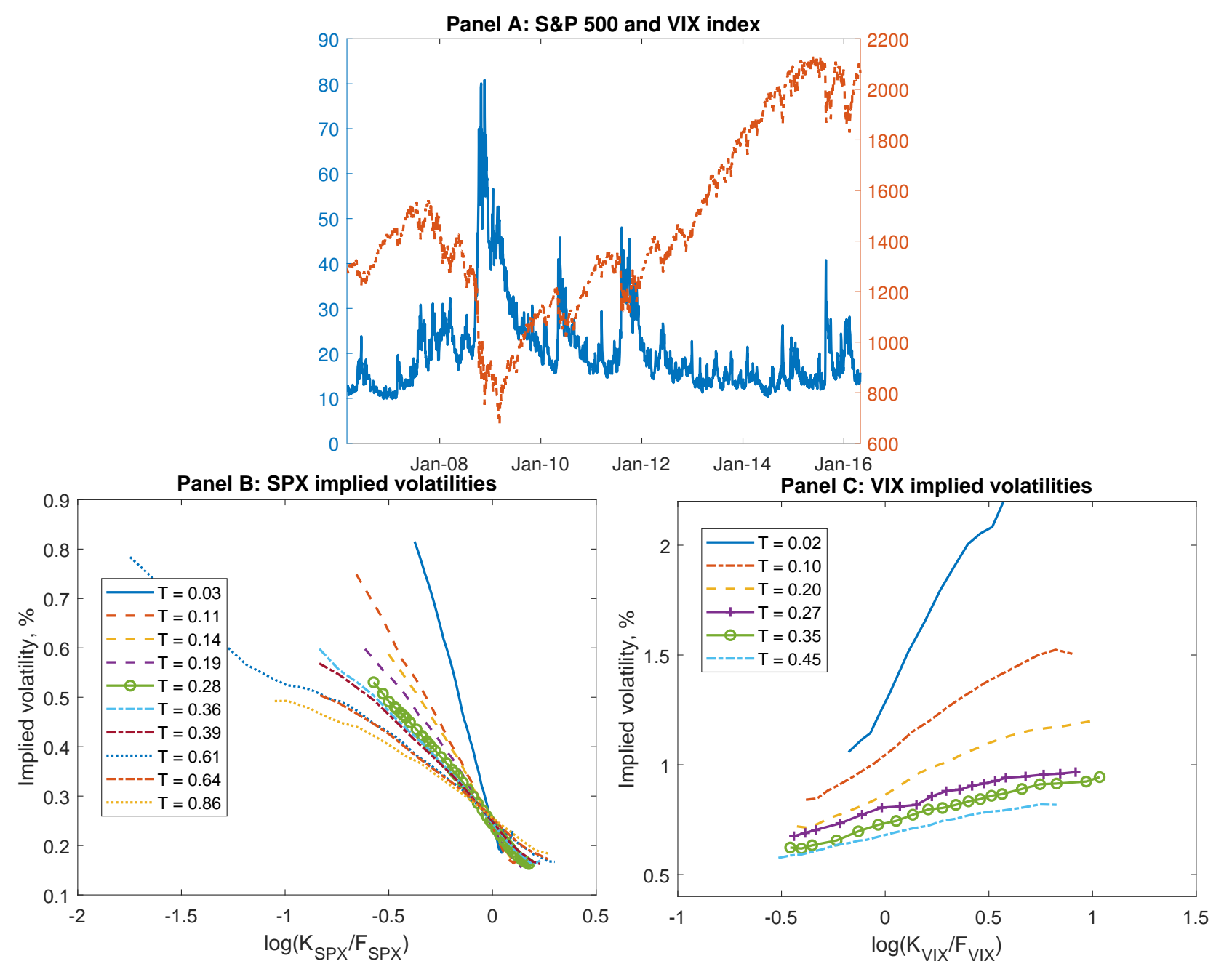

Fig. 1. This figure illustrates some characteristics of the S\&P 500 and VIX markets. Panel A plots the times series of S\&P 500 (dashed curve) and VIX (solid curve) indexes from March 1st, 2006 to April 30th, 2016. Panels B and C represent the implied volatilities of S\&P 500 options and VIX options on May 10 2010, as a function of log-moneyness. The maturities $T$ are quoted in years. 

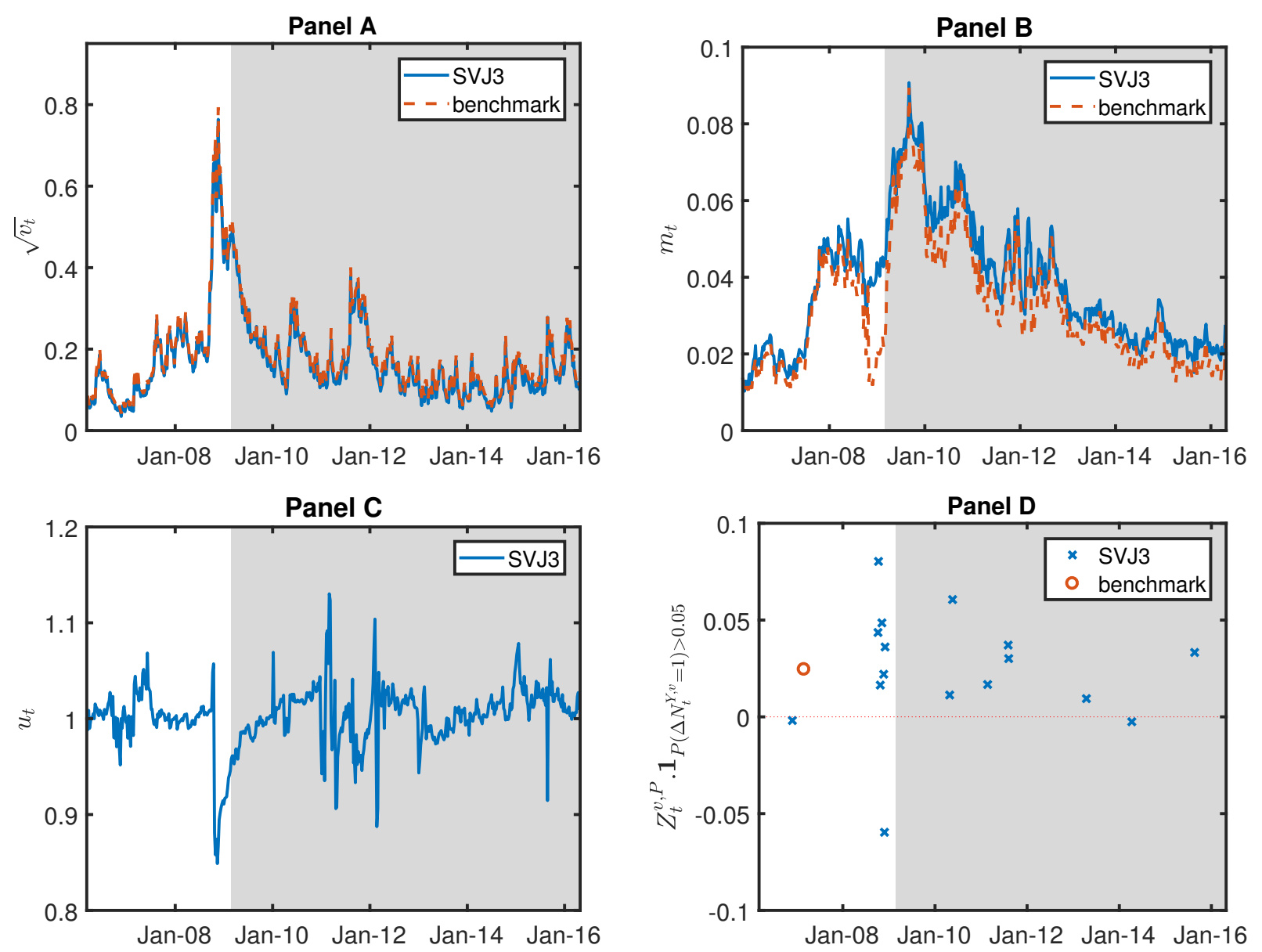

Fig. 2. Panels $\mathrm{A}$ and $\mathrm{B}$ plot the filtered trajectories of the latent volatility processes $v$ and $m$ for the SVJ3 and the benchmark model (these models' dynamics are given respectively in (1)-(3) and (11)), including VIX options in the estimation dataset, from March 2006 to April 30, 2016. Panel C represents the filtered trajectory of the jump intensity factor $u$ and Panel D displays the filtered size of jumps in the variance process $v$, when the probability of jumps exceeds $5 \%$. The shaded part of the graph represents the out-of-sample period, from March 1, 2009 until the end of April 2016. 

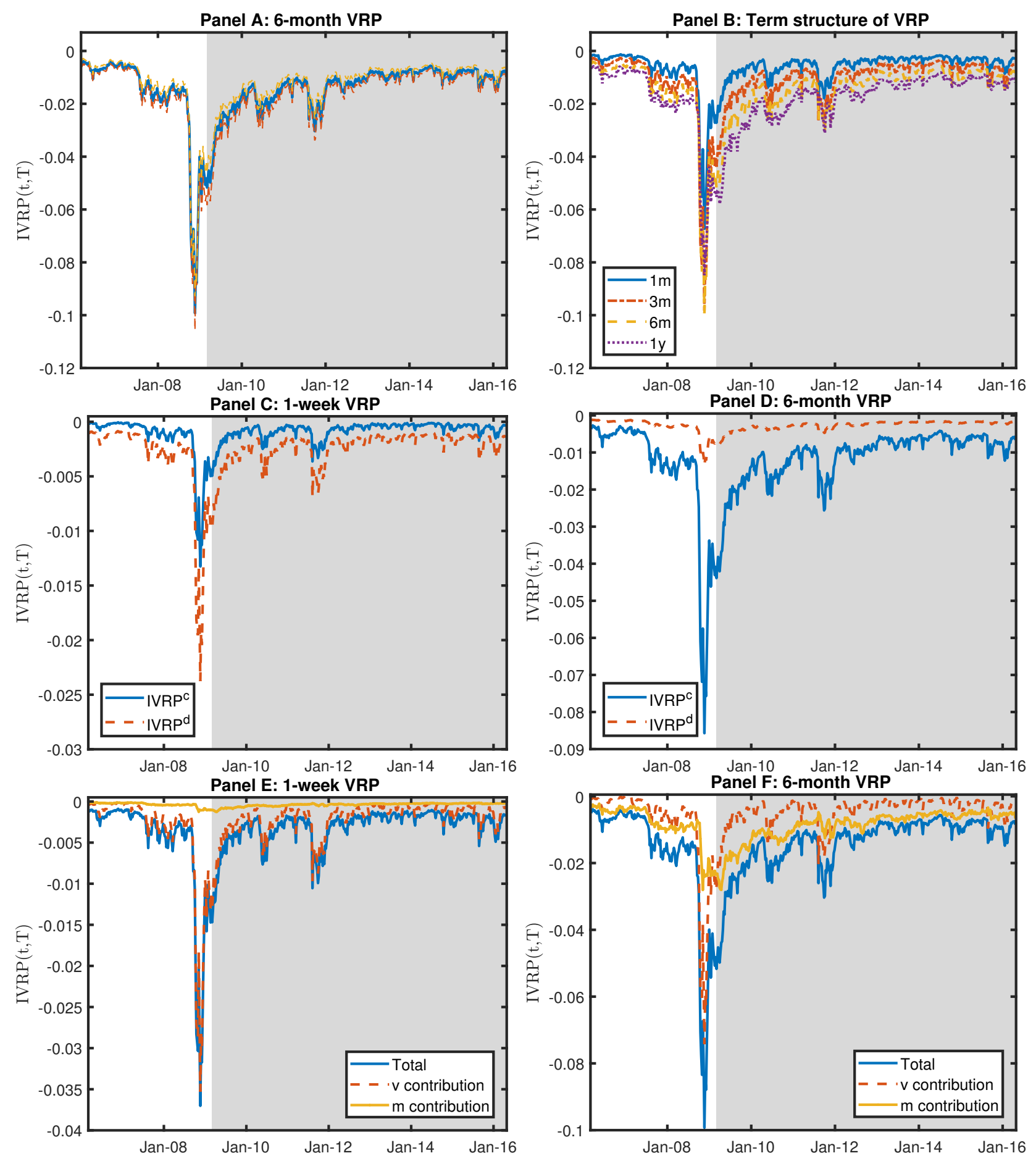

Fig. 3. Integrated variance risk premia (VRP) when estimating the SVJ3 model (dynamics given in (1)-(3)) using the full dataset of S\&P 500 options, VIX options and their underlying levels. Panel A plots the six-month VRP and its $90 \%$ confidence interval, conditional on parameter estimates. Panel $\mathrm{B}$ plots the VRP for different maturities. Panels $\mathrm{C}$ and $\mathrm{D}$ decompose the one-week and six-month VRP into their continuous and discontinuous components. Panels E and F plot the contribution of the latent factors $v$ and $m$ to the one-week and six-month VRP. The shaded parts of the graphs represent the out-of-sample period from March 1, 2009 until the end of April 2016. 

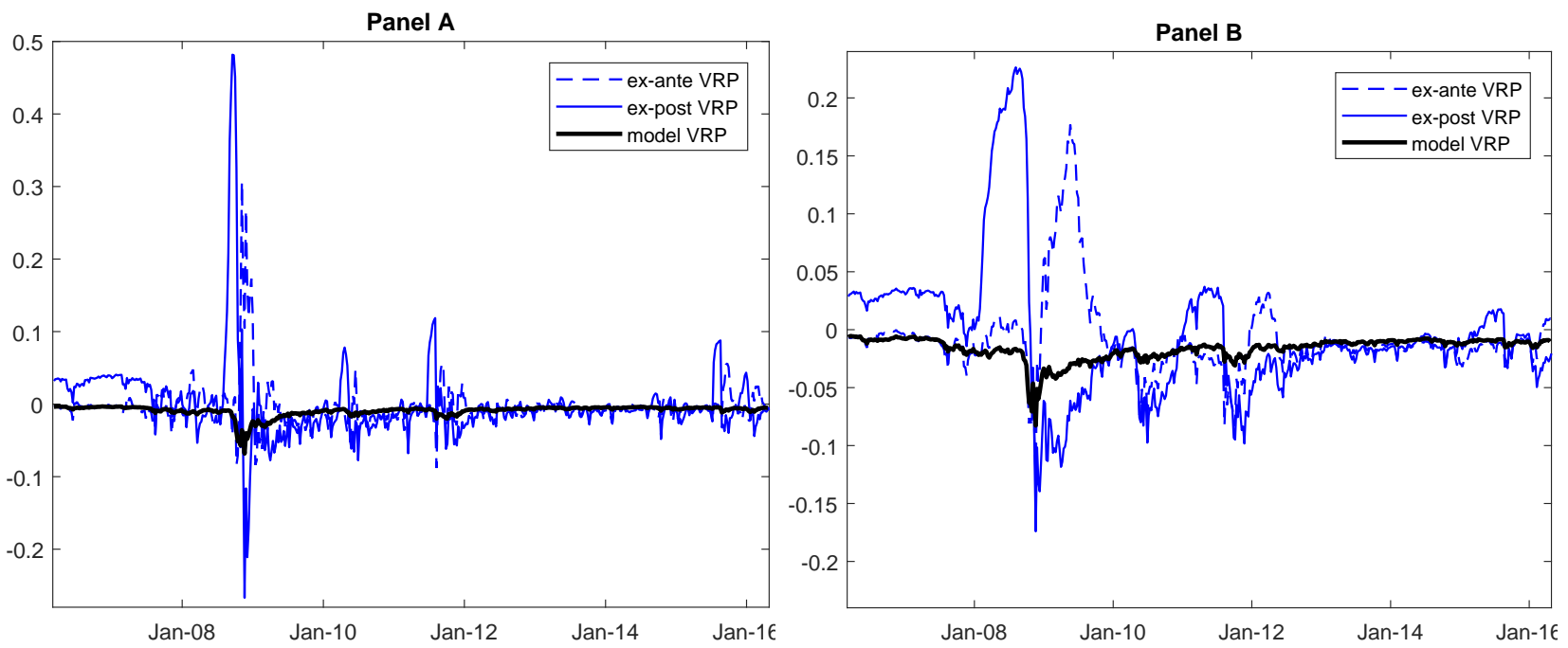

Fig. 4. Integrated variance risk premia (VRP) when estimating the SVJ3 model (dynamics given in (1)-(3)) using the full dataset including VIX options (thick solid line), compared to the ex-ante model-free estimate of the VRP, computed as the difference between the past realized variance and the squared VIX (dashed line), and the approximated ex-post VRP, computed as the difference between the realized variance and the squared VIX (thin solid line). Panel A corresponds to an investment of one month and Panel B to six months. 

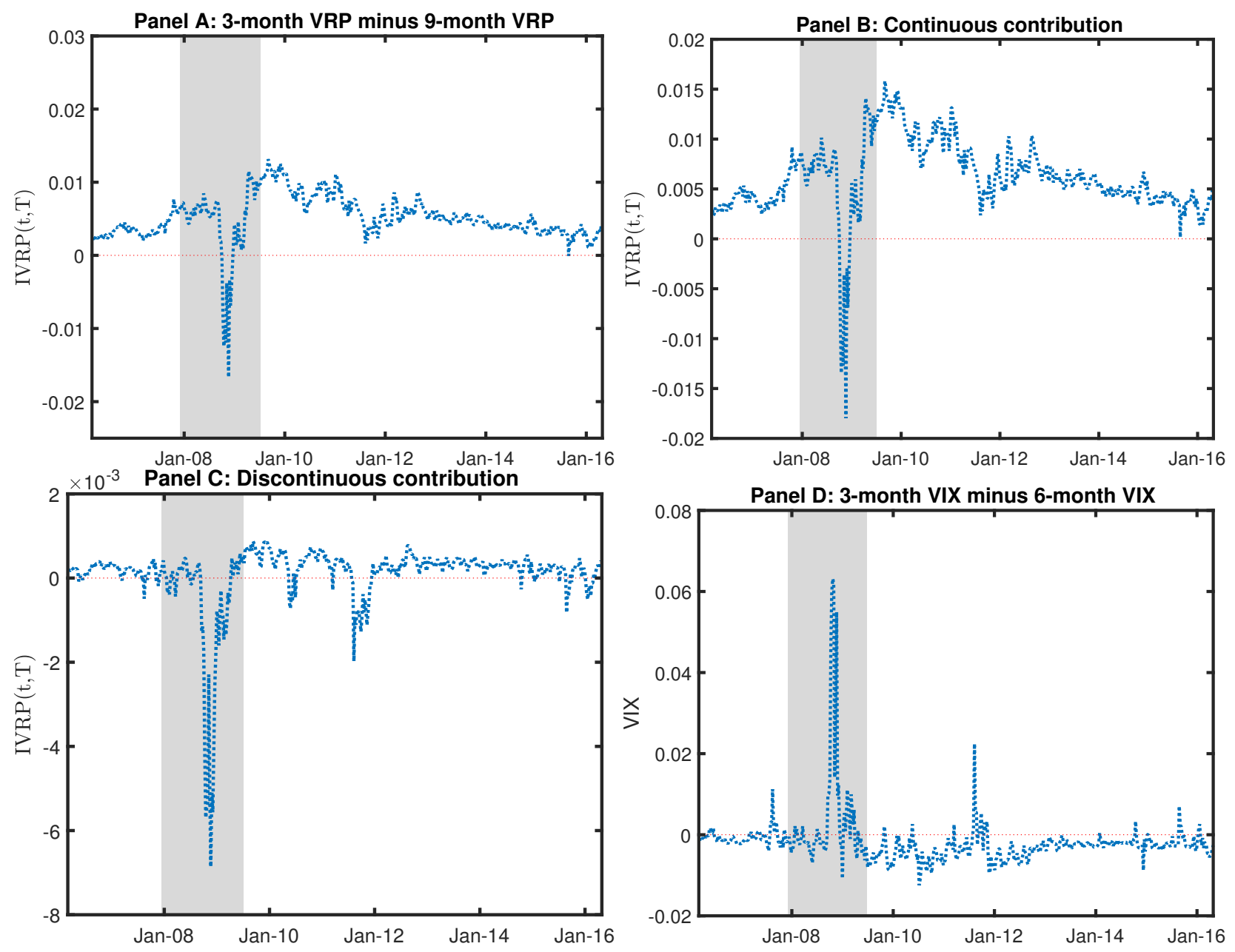

Fig. 5. Panel A displays the total difference between the three-month and the 9-month integrated variance risk premia (VRP) when estimating the SVJ3 model (dynamics given in (1)-(3)) using the full dataset of S\&P 500 options, VIX options and their underlying levels. Panel B plots the contribution of the continuous fluctuations in the VRP to this difference, and Panel $\mathrm{C}$ the contribution of the jumps. Panel D plots the difference between the three-month and the six-month VIX constructed from S\&P 500 options. The shaded part of the graphs represents the NBER recession from December 2007 until June 2009. 

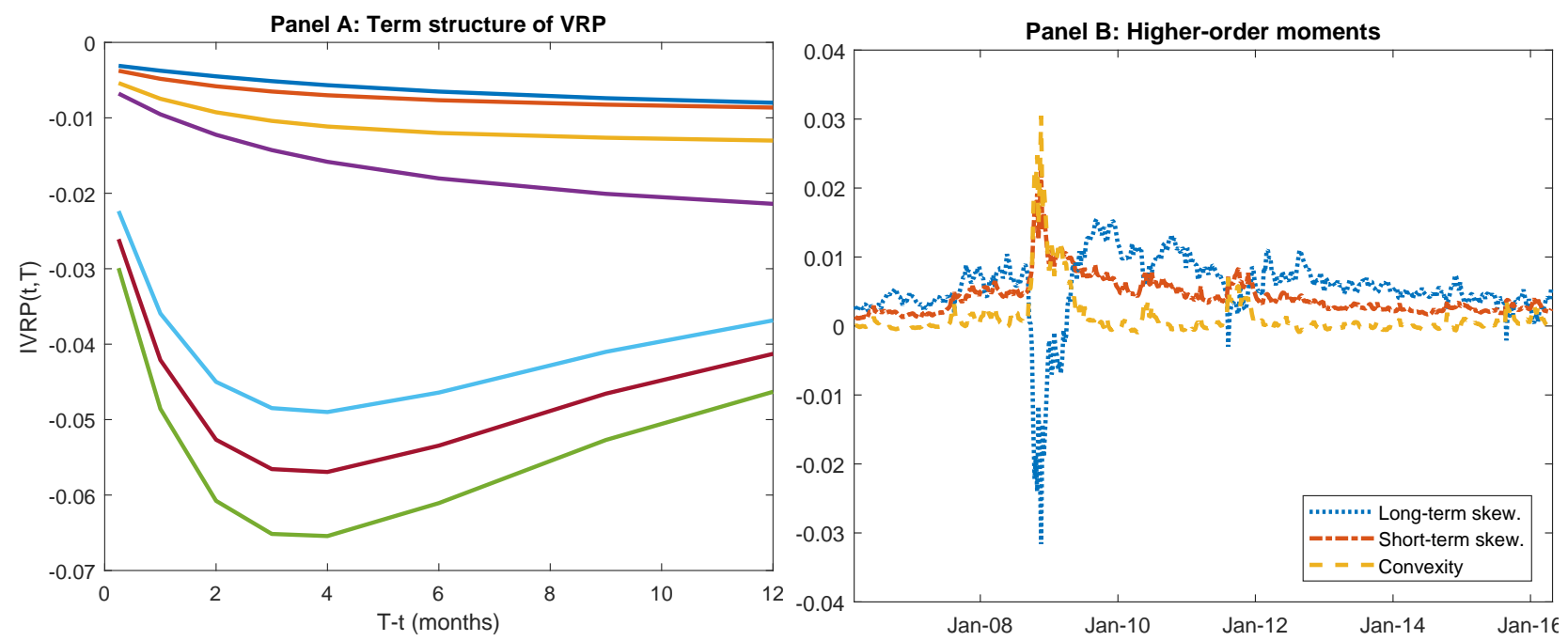

Fig. 6. Panel A represents the term structure of variance risk premium for investment horizons from one month to one year. The VRP is calculated on the following dates, going from the upper curve to the lower one: 3 March 2006, 17 May 2006, 14 June 2006, 18 June 2008, 5 November 2008, 10 December 2008, 22 October 2008. Panel B plots the long- and short-term skewness and the convexity of the term structure of VRP over time. 

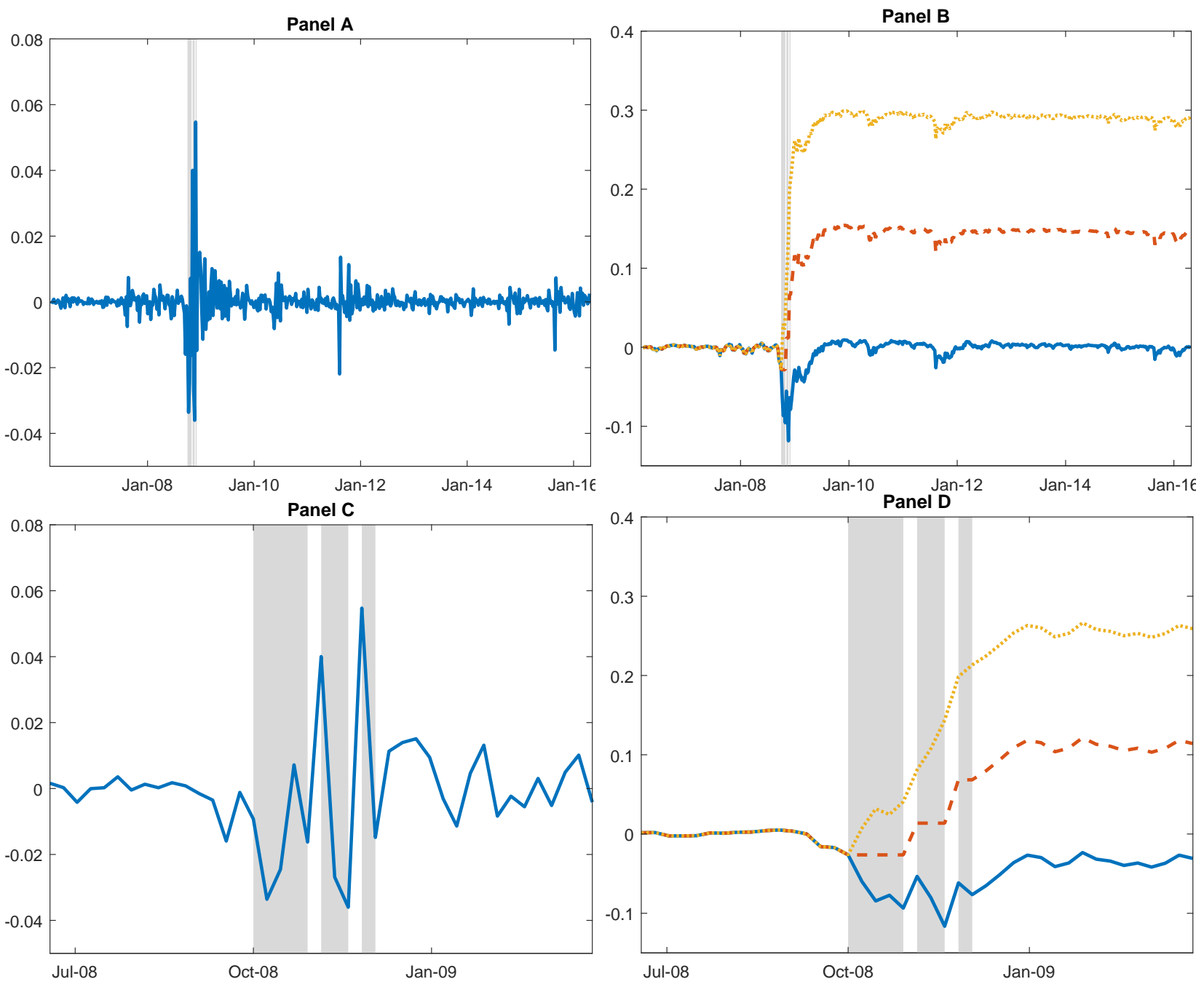

Fig. 7. Panel A represents the returns of a weekly investment in the forward variance with $T_{1}=3$ months and $T_{2}=6$ months. The grey areas indicate the weeks in which there is a switch in the slope of the VRP term structure. Panel B displays the cumulative returns of this investment and the variants that we propose. The plain line (lower line) corresponds to the usual long-short strategy, the dashed line (middle line) to the strategy that is kept on hold whenever there is a switch in the slope of the VRP term structure, and the dotted line (upped line) to the strategy that takes the opposite position of the usual strategy during these periods of switches. Panels $\mathrm{C}$ and $\mathrm{D}$ are zooms of Panels A and B, which focus on the period during which such switches are observed. 

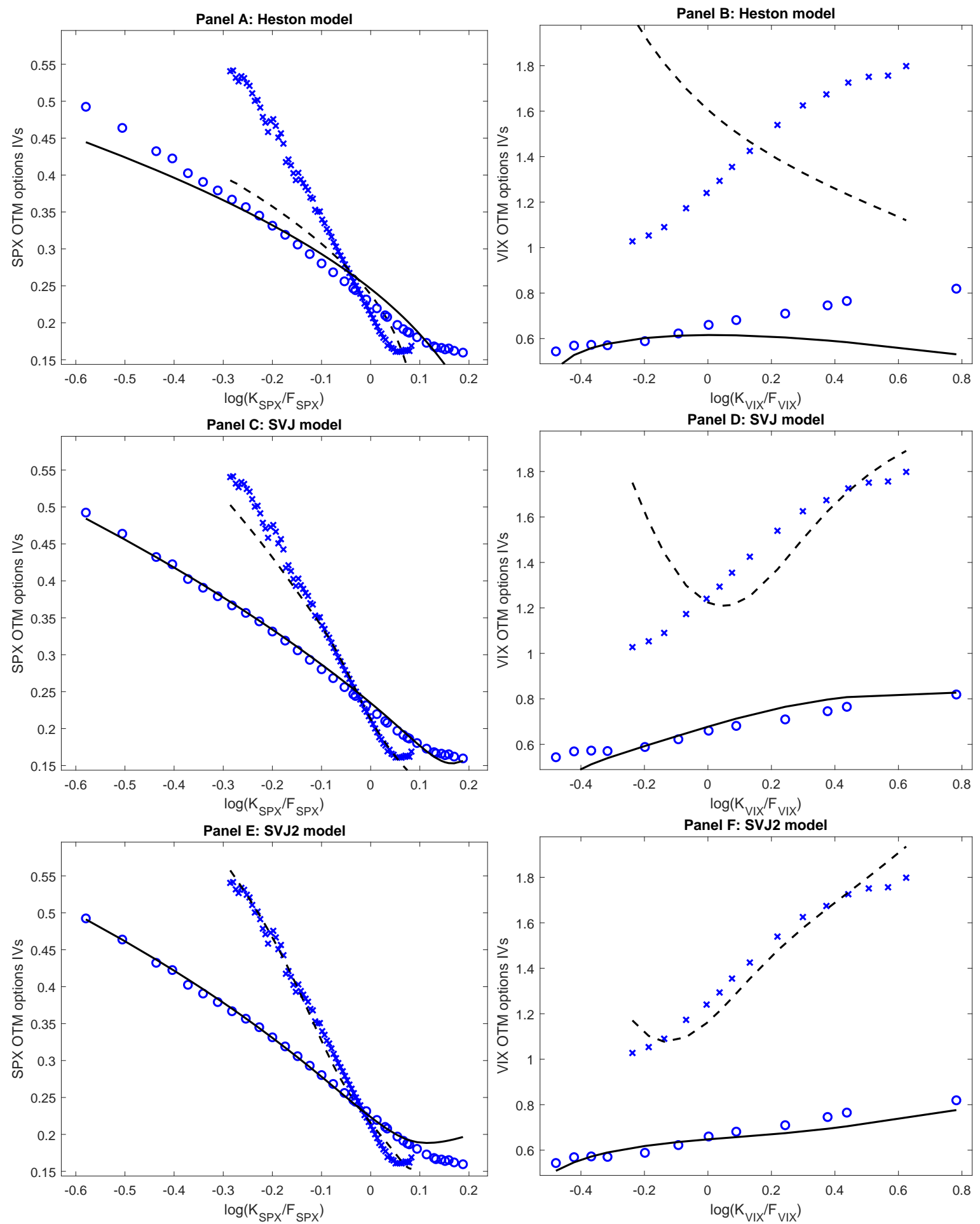

Fig. D.1. This figure represents fitted IVs on May 5, 2010, obtained by a joint calibration on the S\&P 500 and VIX options. Circles represent the market IV for $T=0.05$ (S\&P 500) and $T=0.04$ (VIX). Crosses represent the market IV for $T=0.3$ (S\&P 500) and $T=0.36$ (VIX). The dashed line corresponds to the model fit for $T=0.05$ (S\&P 500) and $T=0.04$ (VIX). The solid line corresponds to the model fit for $T=0.3$ (S\&P 500) and $T=0.36$ (VIX). Panels A (S\&P 500) and B (VIX) plot the model IVs for the Heston model, Panels C and D for the SVJ model, and Panels E and F for the SVJ2 model. 

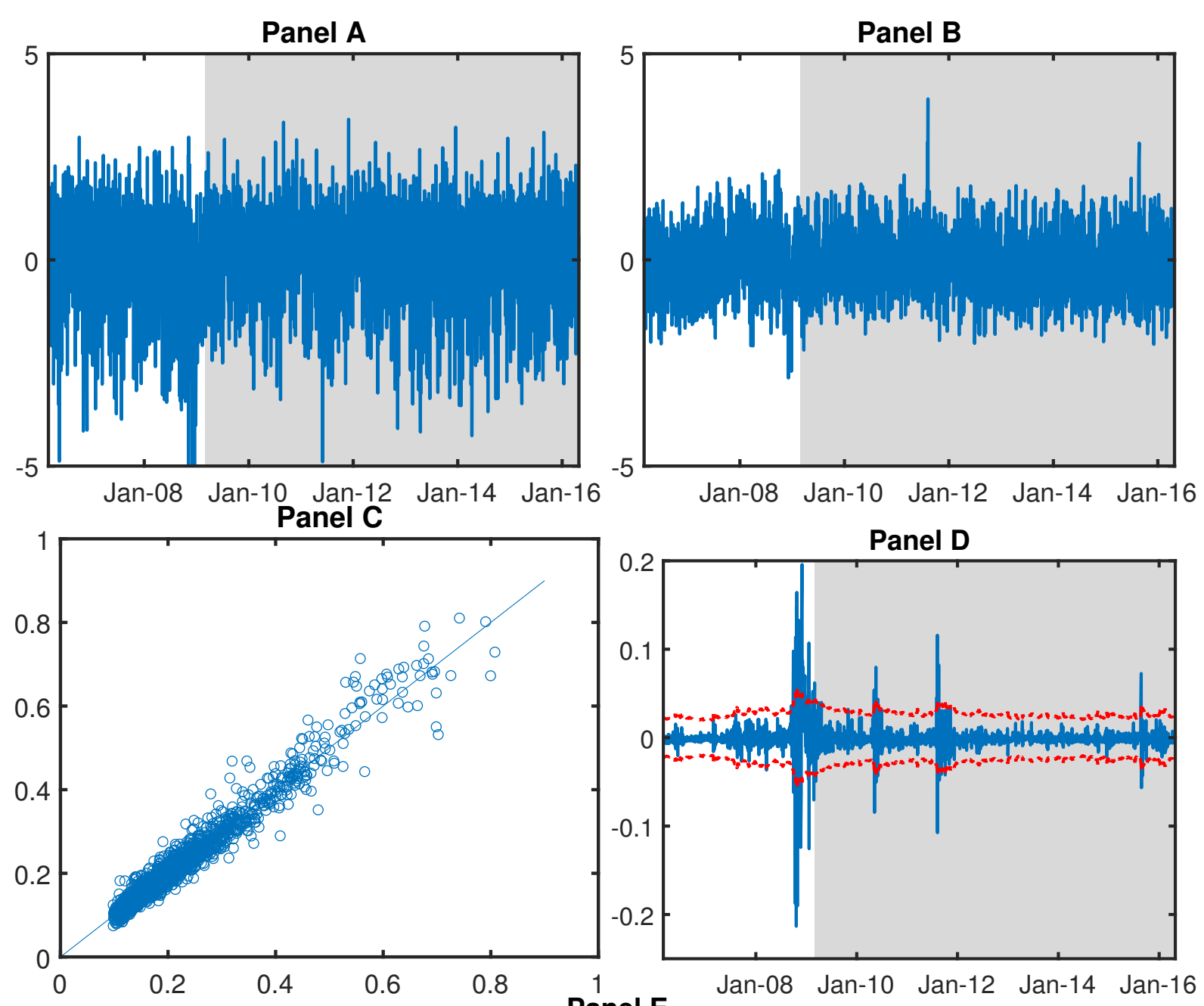

Panel D
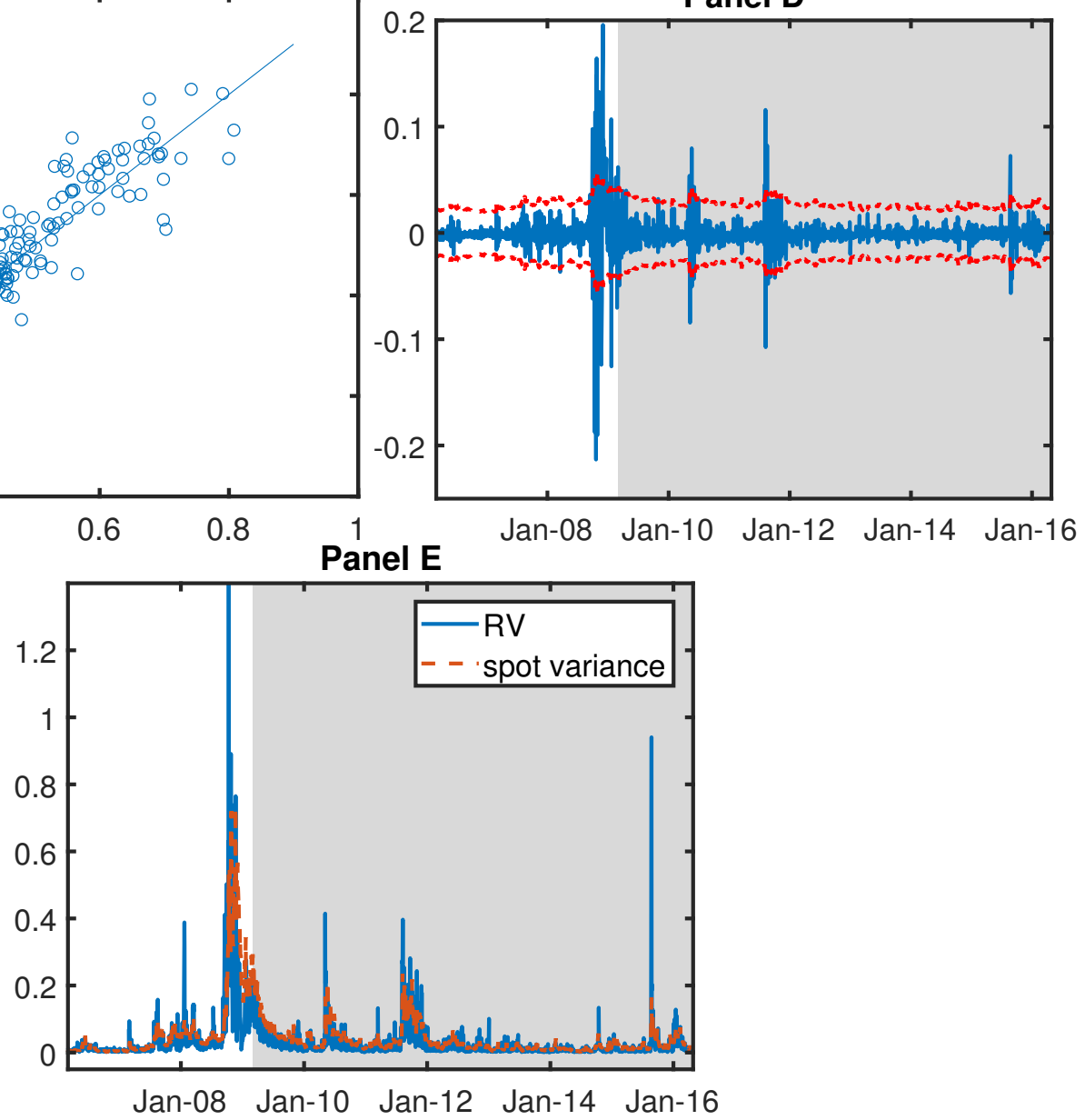

Fig. G.1. This figure provides model diagnostics to evaluate the SVJ3 specification, when the estimation dataset includes VIX options. Panels A and B represent the filtered Brownian motions driving the dynamics of the S\&P 500 returns and their variance factor $v$. Panel $\mathrm{C}$ is a scatterplot comparing model-implied VIX values to the data across the (in- and out-of-sample) time series. Panel D plots the error between the true VIX squared and the filtered value throughout time. Panel E represents the realized variance computed from high-frequency data and the estimated trajectory of the model-implied quadratic variation under $\mathbb{P}_{75}$ 

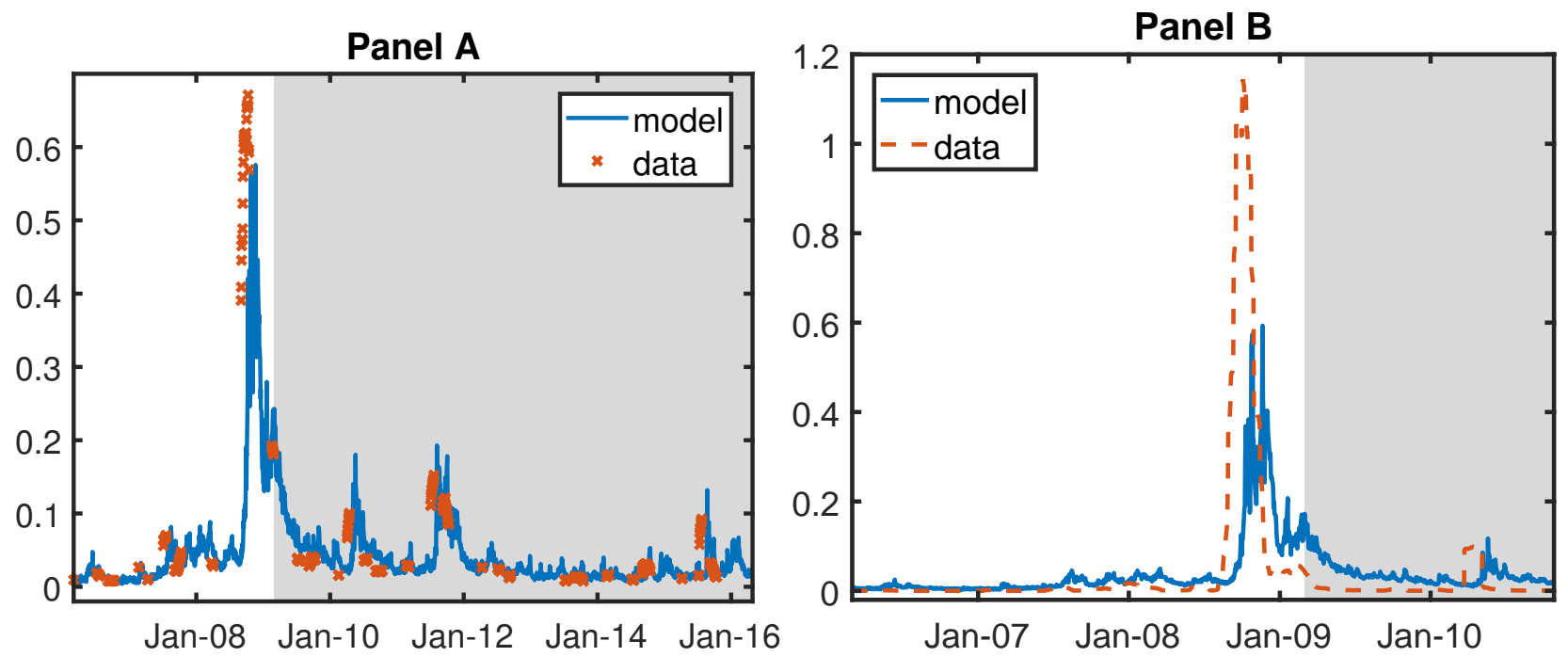

Fig. G.2. This figure compares, for every month, future measures of variation calculated from high frequency returns, to the model-implied expectation of their counterpart. Panel A represents the total variance realized during each month, versus the model $\mathbb{P}$-expectation of the total variance at the beginning of the month. Panel B represents the ex-post tri-power quarticity versus the expectation of the integrated quarticity (based on data available up to end of 2010). 